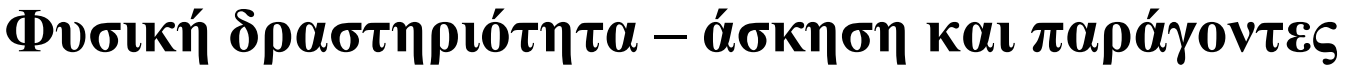

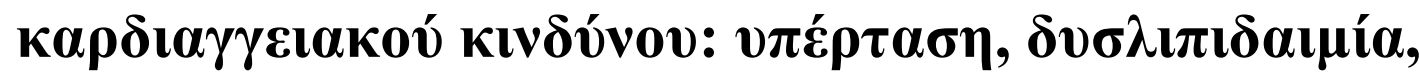 $\pi \alpha \chi v \sigma \alpha \rho \kappa i ́ \alpha$
}

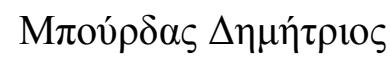

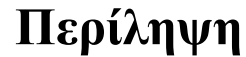

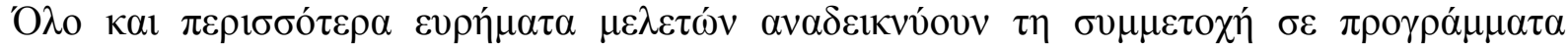

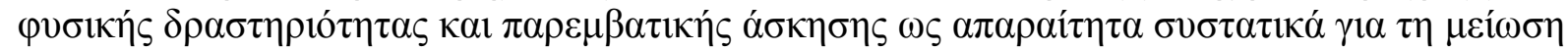

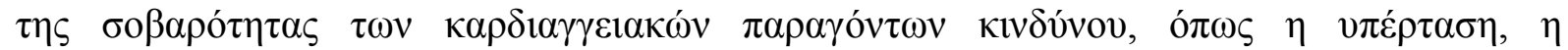

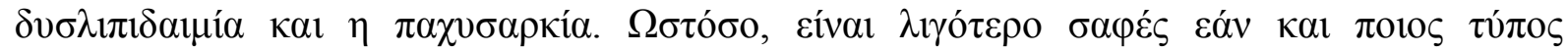

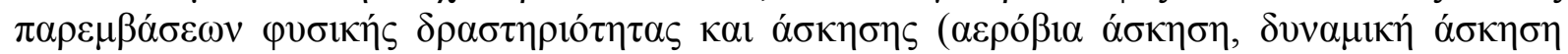

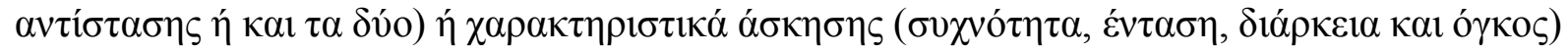
$\theta \alpha$ a

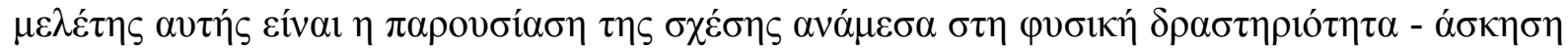

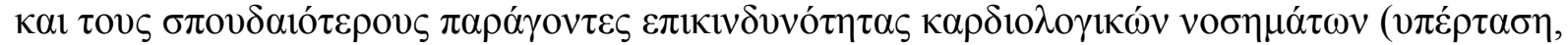

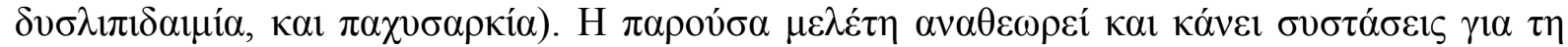

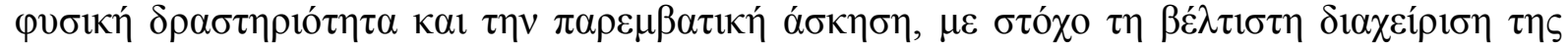

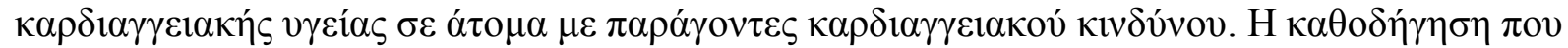

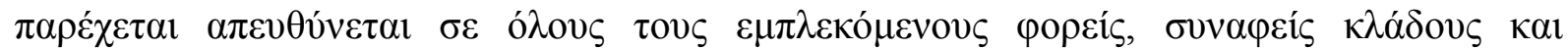

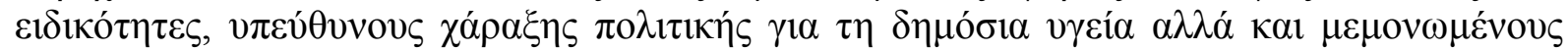

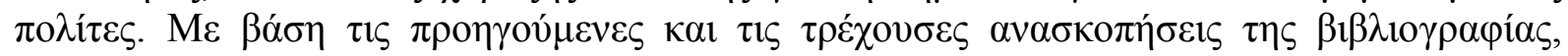

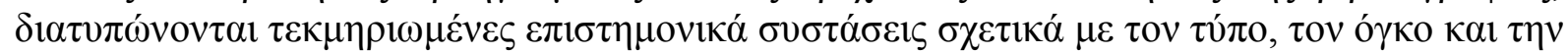

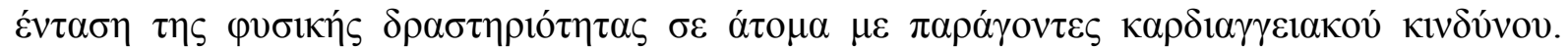

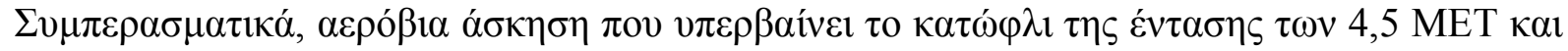

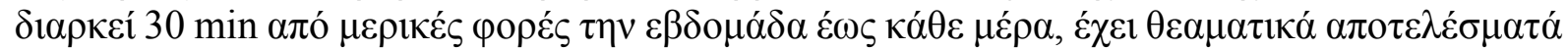

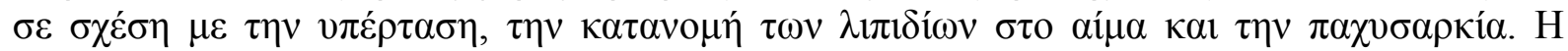

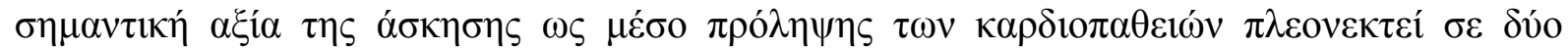

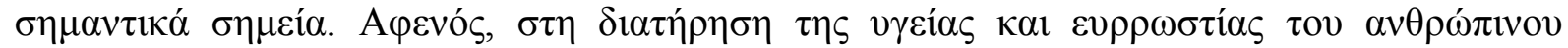

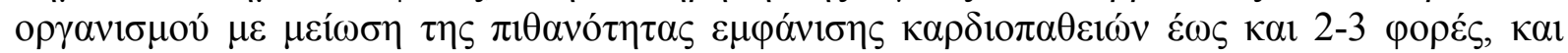

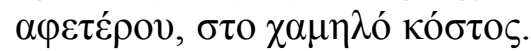

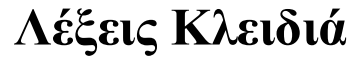

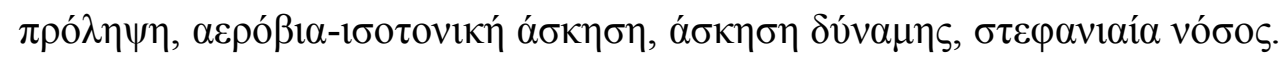

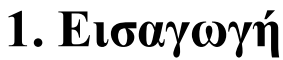

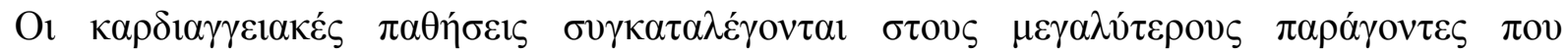

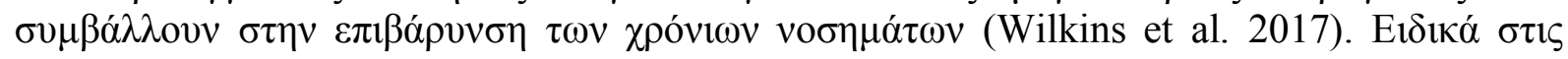

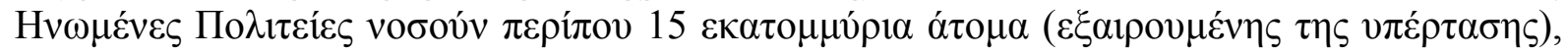

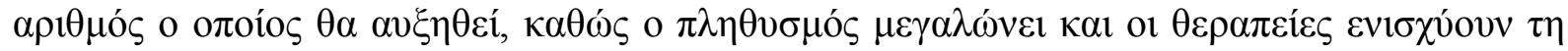

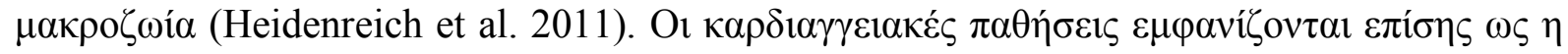

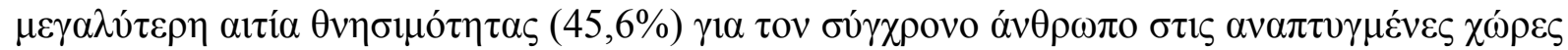

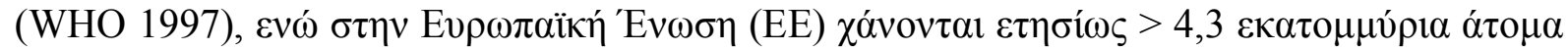

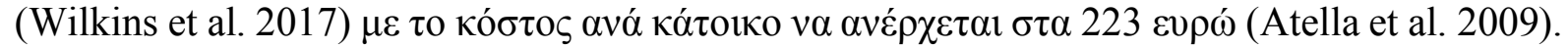

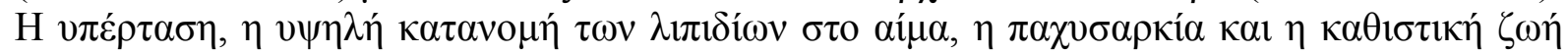




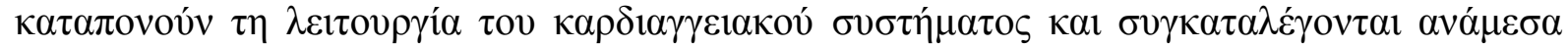

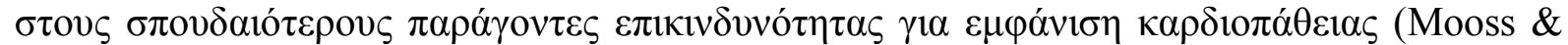
Gordon 2001).

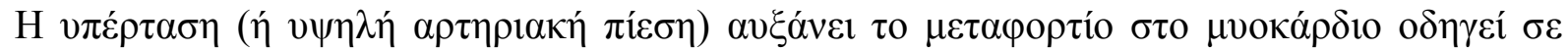

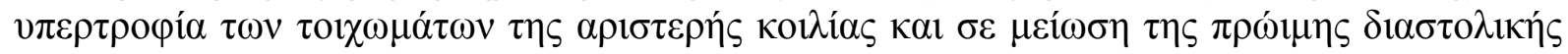

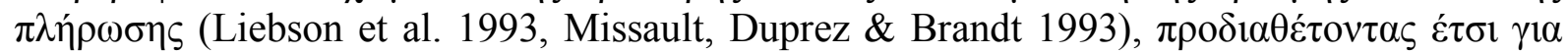
$\varepsilon \mu \varphi \alpha ́ v i \sigma \eta ~ \kappa \alpha \rho \delta ı \pi \alpha ́ \theta \varepsilon ı 1 \alpha \varsigma$ (Kannel \& McGee 1979, Stamler, Neaton \& Wentworth 1993). H

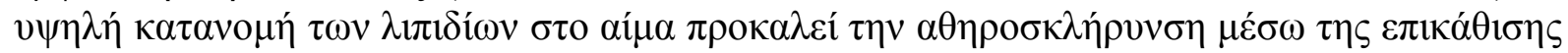

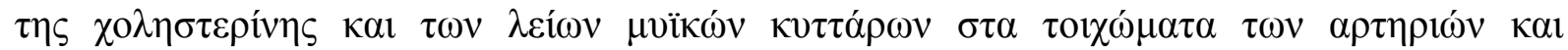

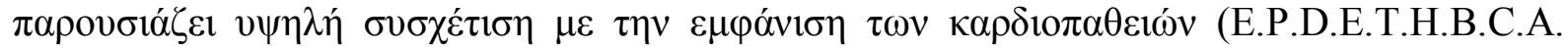

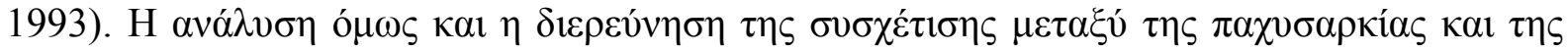

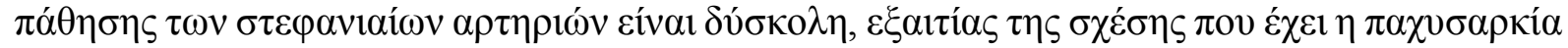

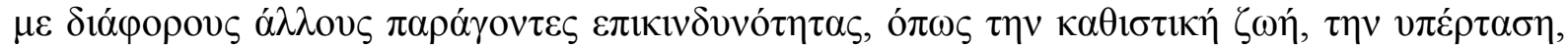

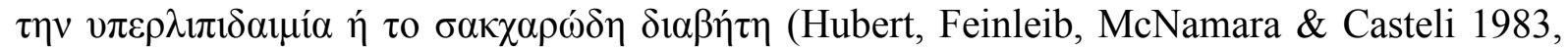

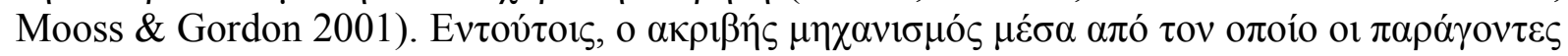

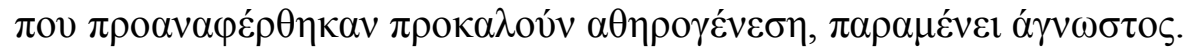

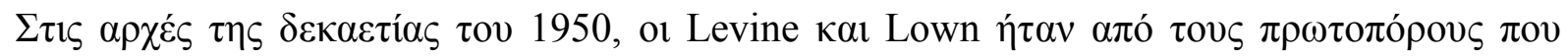

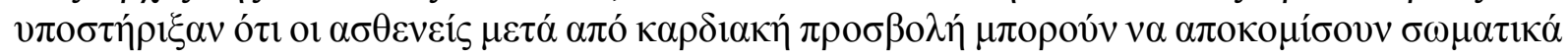

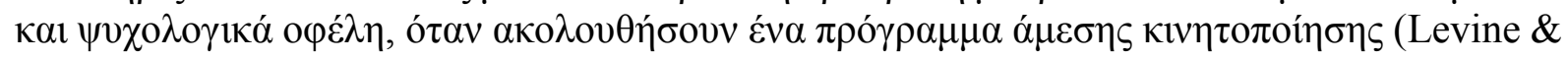

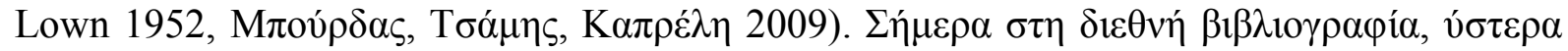

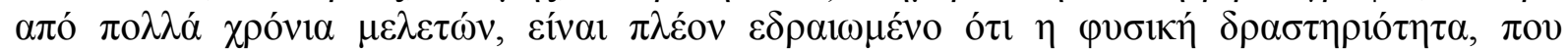

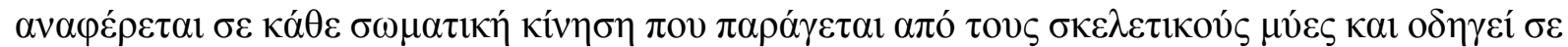

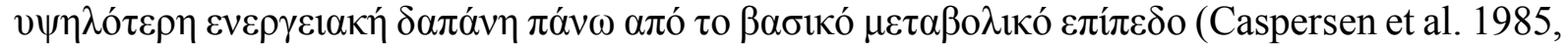

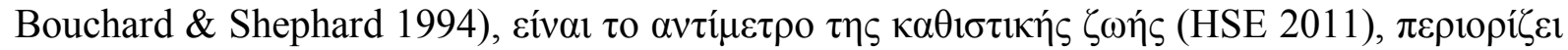

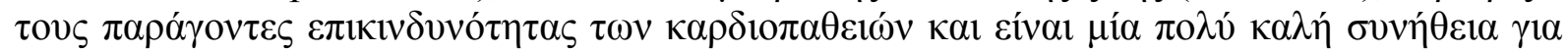

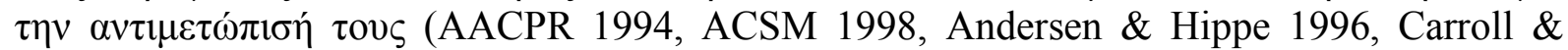
Dudfield 2004, Dubbert et al. 2002, MacKnight 2003, Oguma \& Shinoda-Tagawa 2004, Taylor

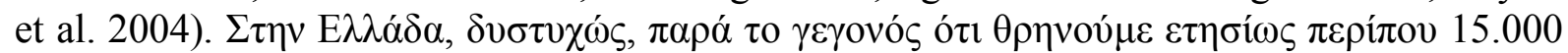

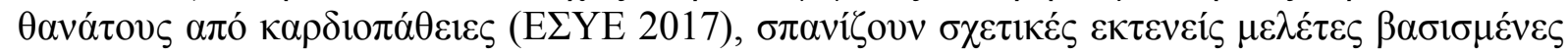

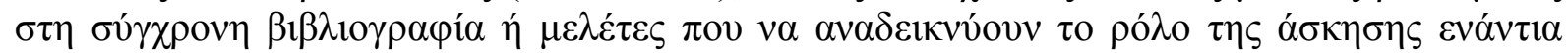

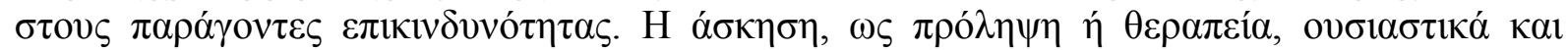

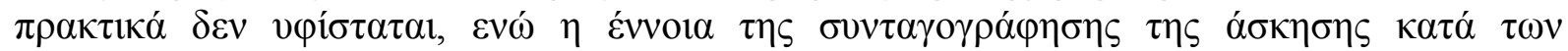

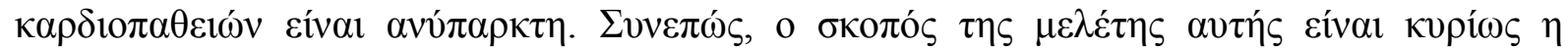

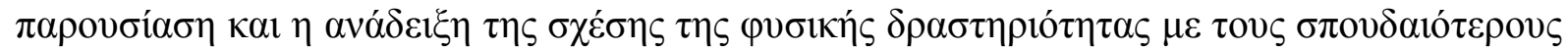

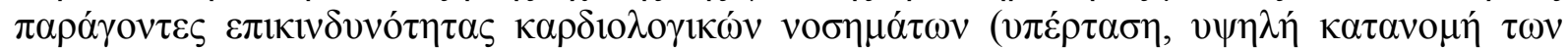

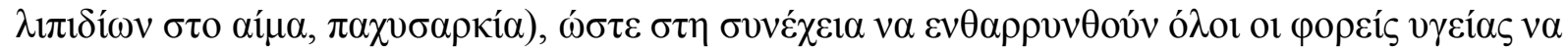

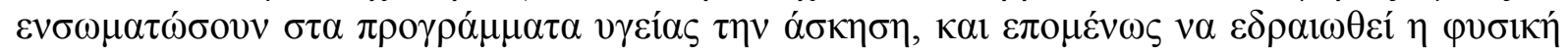

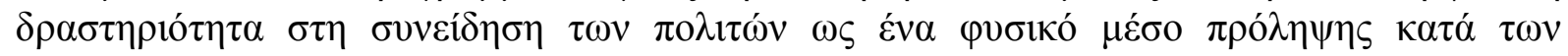

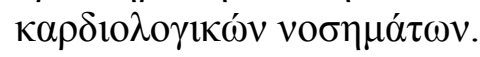

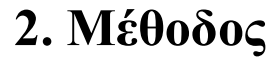

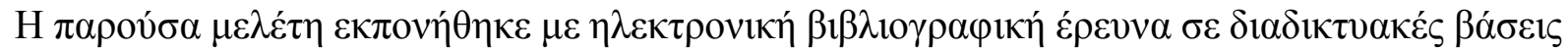

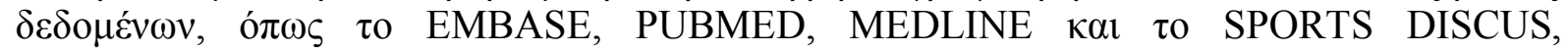

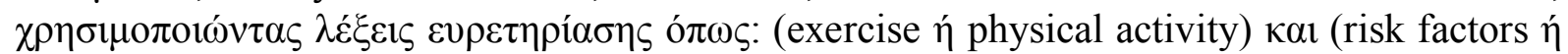
hypertension $\eta$ dislipidemia $\dot{\eta}$ hyperlipidemia $\dot{\eta}$ obesity) $\kappa \alpha$ (heart $\dot{\eta}$ cardiovascular disease).

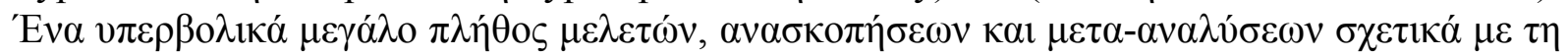

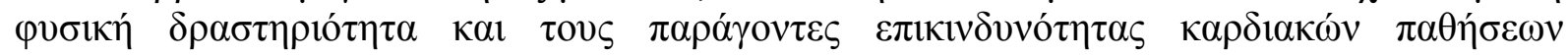

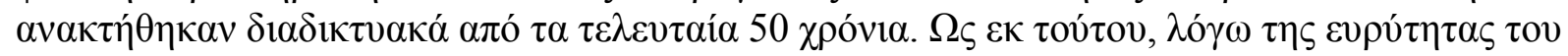

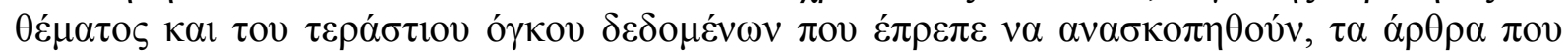




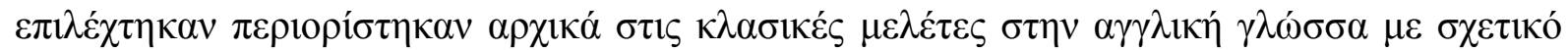

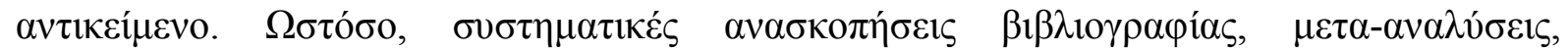

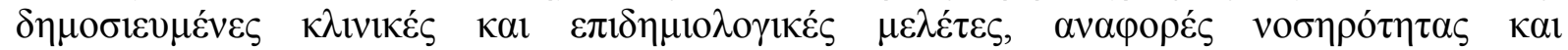

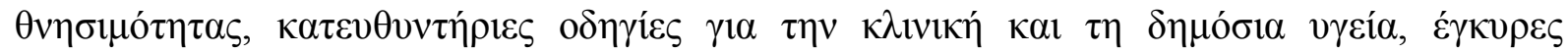

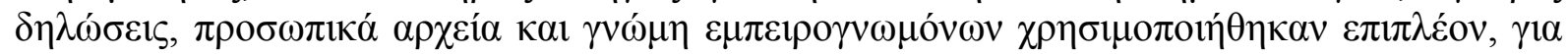

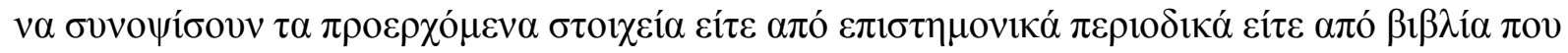

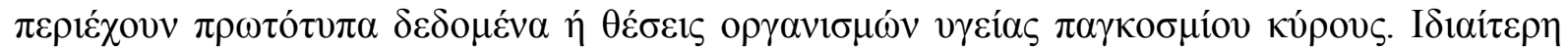

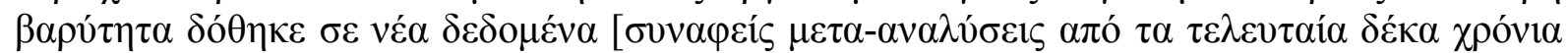

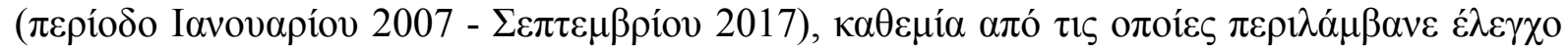

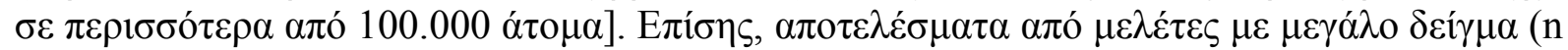

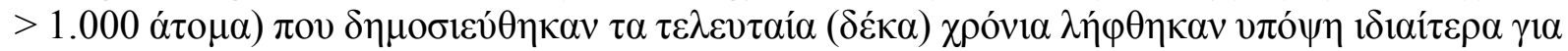

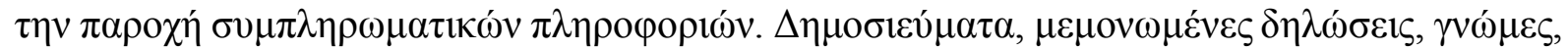

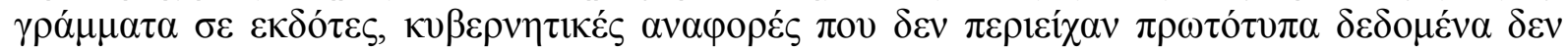
$\sigma v \mu \pi \varepsilon \rho \imath \lambda \eta \dot{\eta} \theta \eta \sigma \alpha \nu \sigma \tau \eta \mu \varepsilon \lambda \varepsilon \dot{\varepsilon} \eta$.

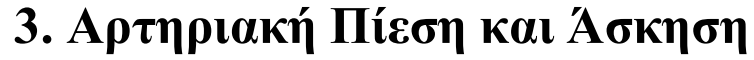

\section{Y $\boldsymbol{\varepsilon} \rho \tau \alpha \sigma \eta$}

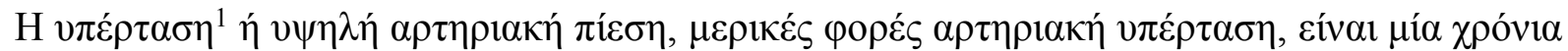

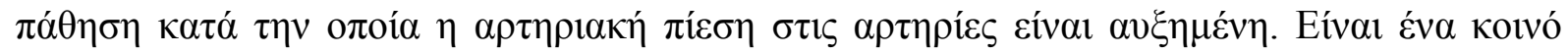

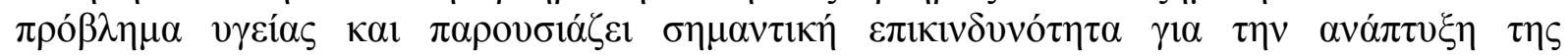

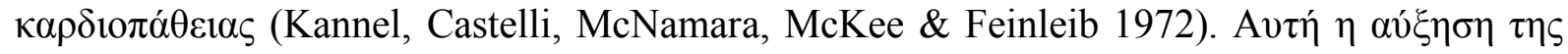

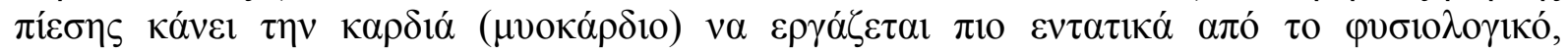

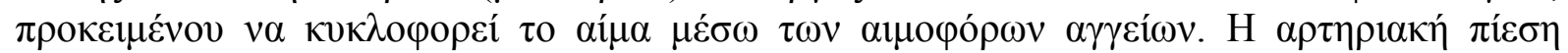

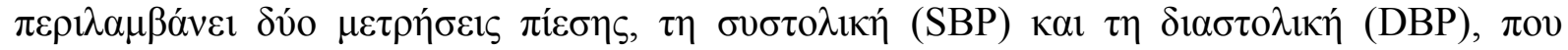

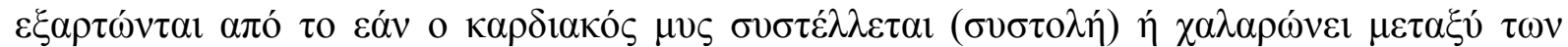

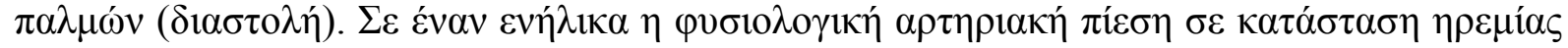

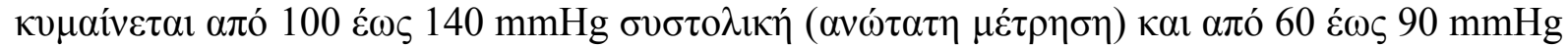

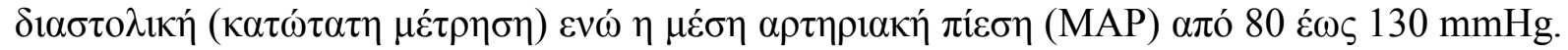

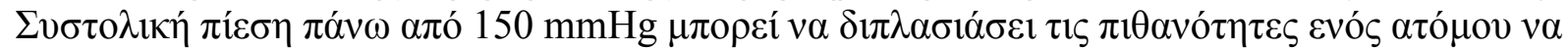

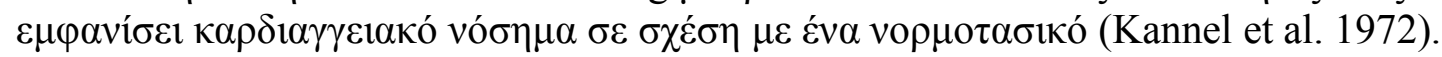

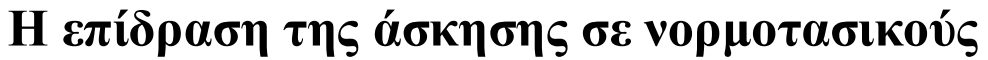

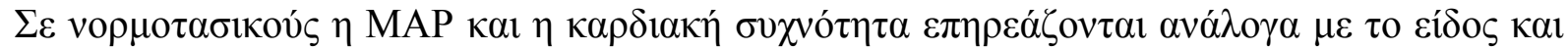

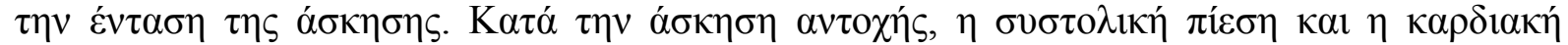

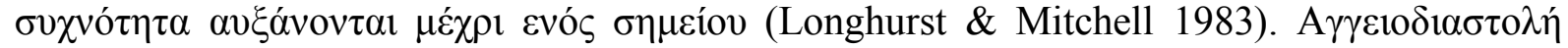

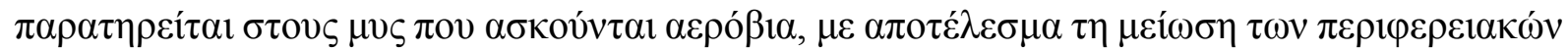

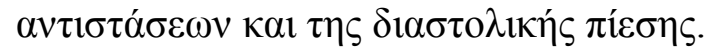

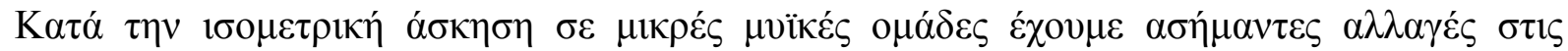

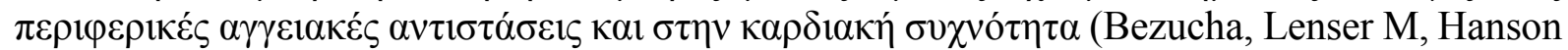

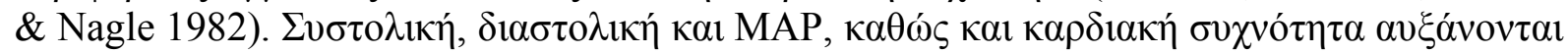

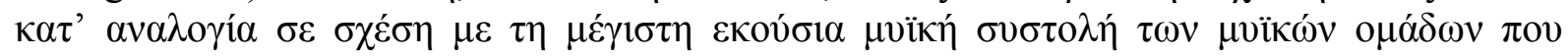

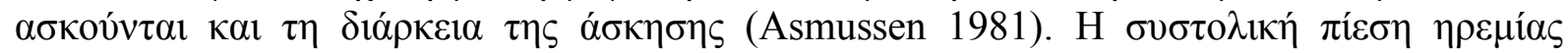

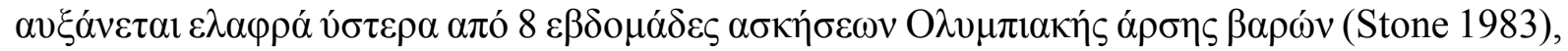

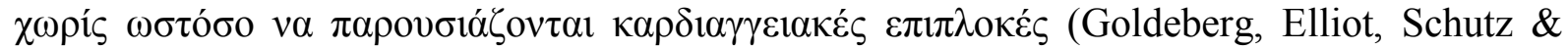

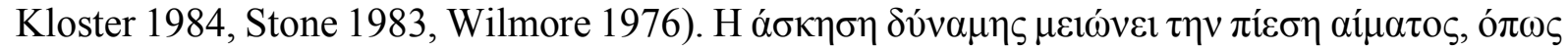

\footnotetext{
${ }^{1} \sigma v \sigma \tau \circ \lambda \iota \kappa \eta ́ ~ \pi i ́ \varepsilon \sigma \eta \geq 140 \mathrm{mmHg}, \delta \iota \alpha \sigma \tau о \lambda \iota \kappa \eta ́ ~ \pi i ́ \varepsilon \sigma \eta \geq 90 \mathrm{mmHg}$ (T.S.R.J.N.C.P.D.E.T.H.B.P. 1997)
} 


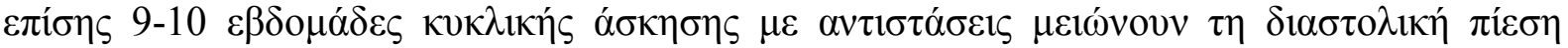
(Harris \& Holly 1987, Wilmore 1976).

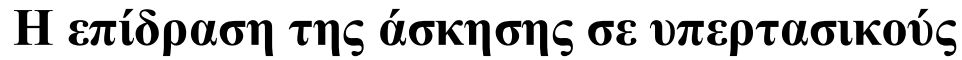

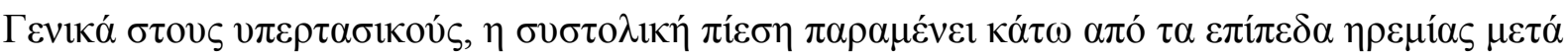

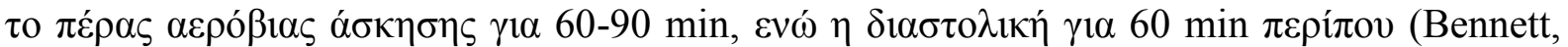

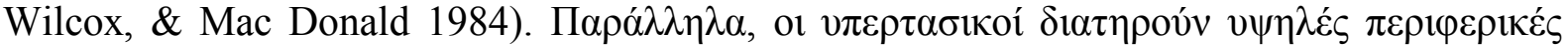

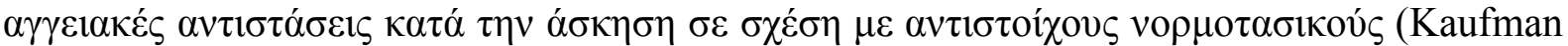

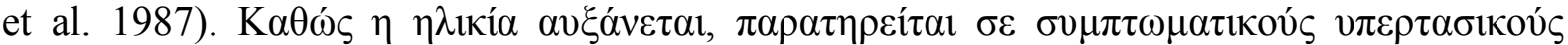

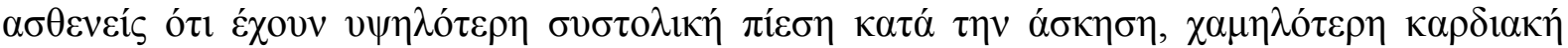

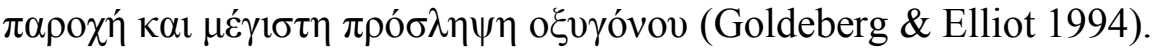

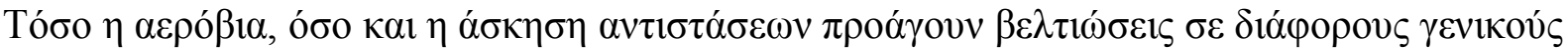

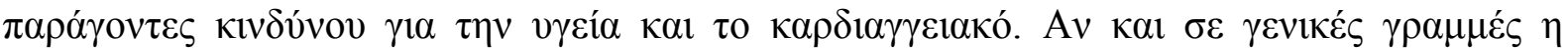

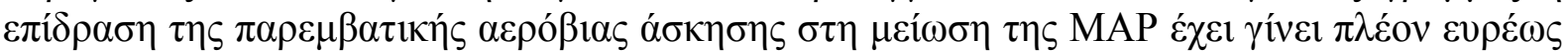

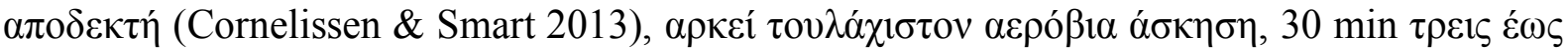

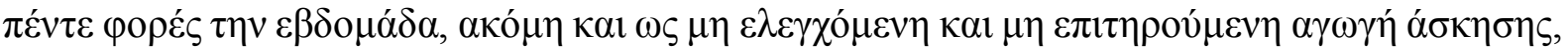

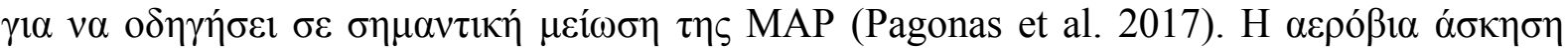

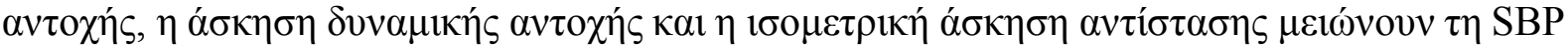

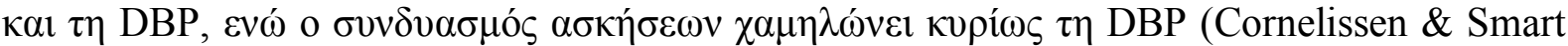

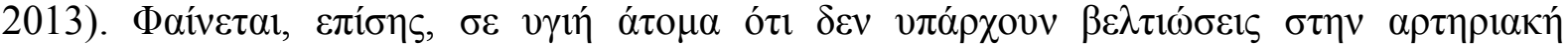

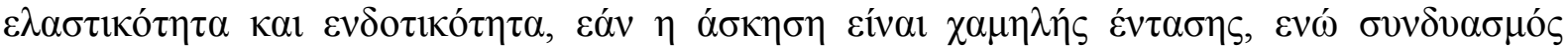

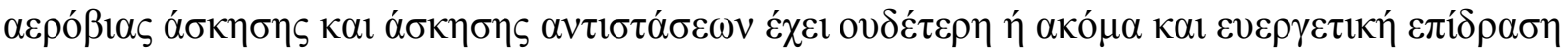

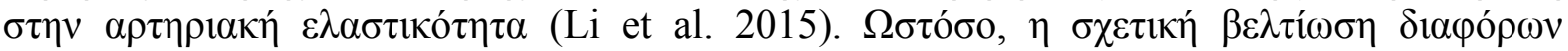

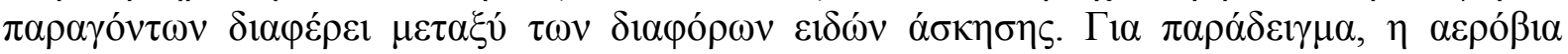

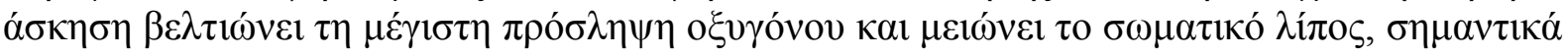

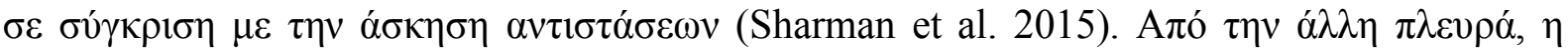

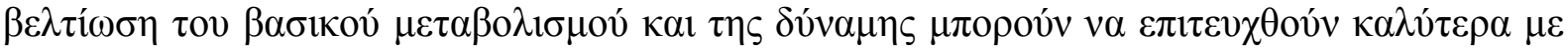

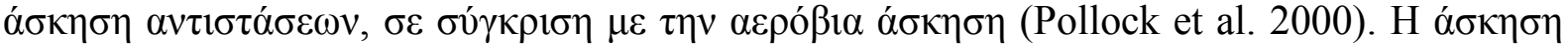

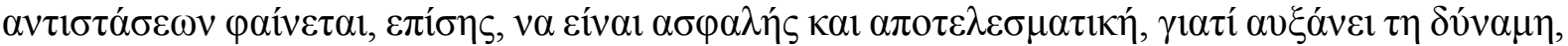

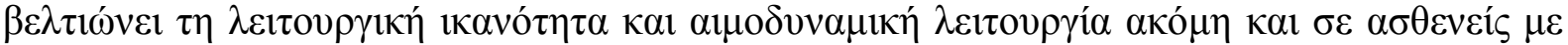

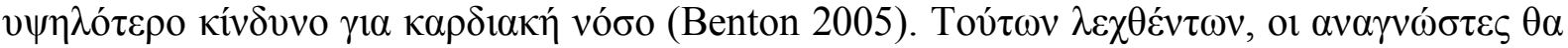

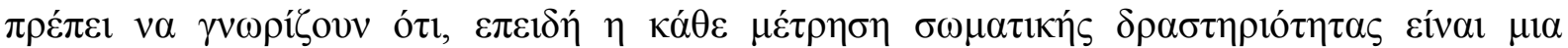

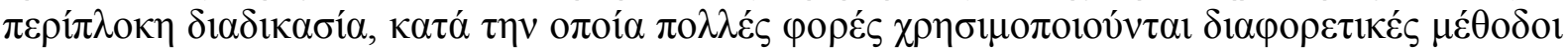

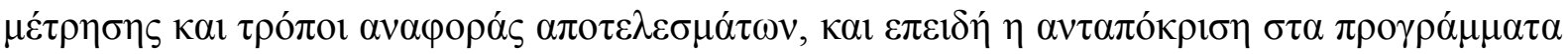

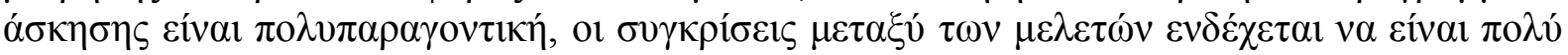
$\delta$ $\sigma \kappa о \lambda \varepsilon \varsigma$.

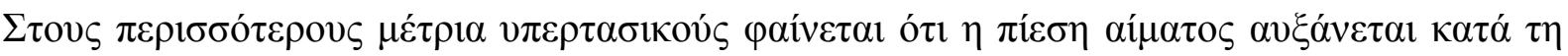

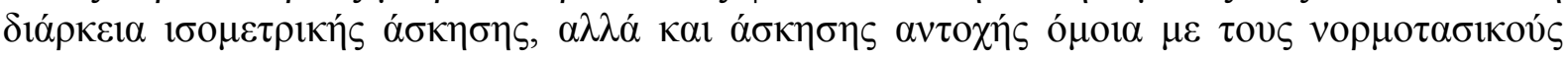
(Bennett, Wilcox, \& Mac Donald 1984, Kaufman, Hughson \& Shaman 1987). Ta $\delta \varepsilon \delta o \mu \varepsilon ́ v \alpha$,

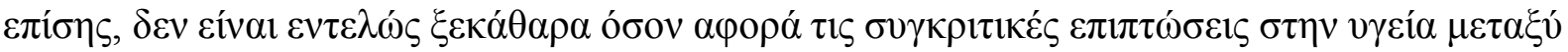

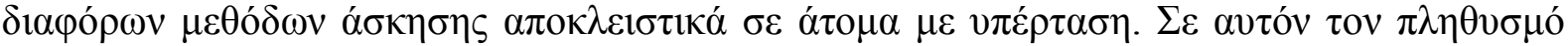

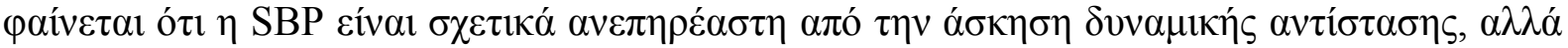

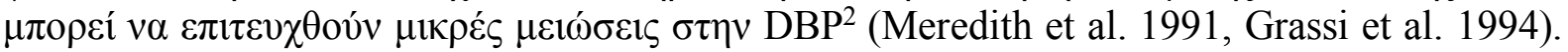

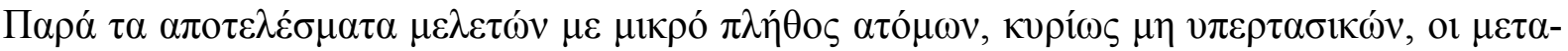

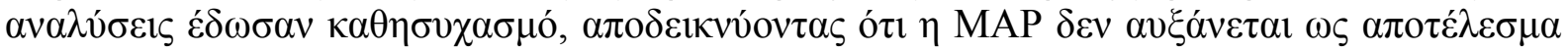

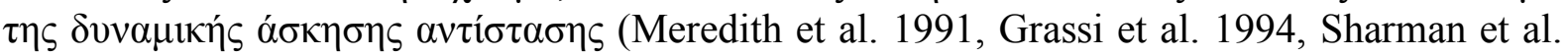

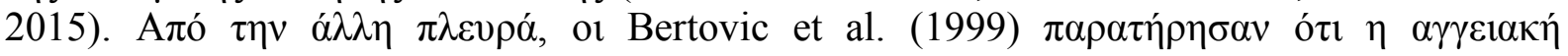

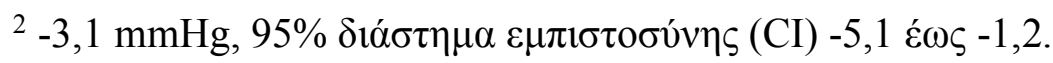




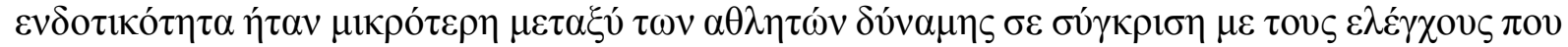

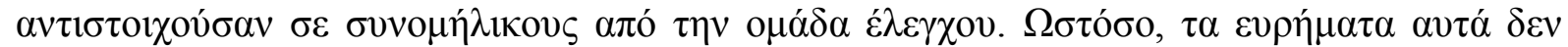

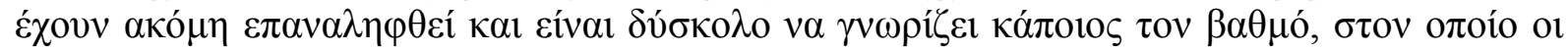

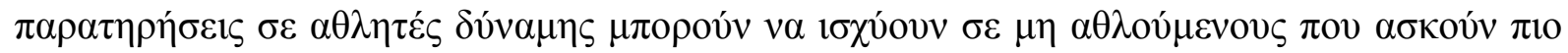

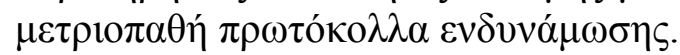

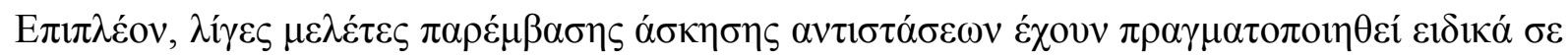

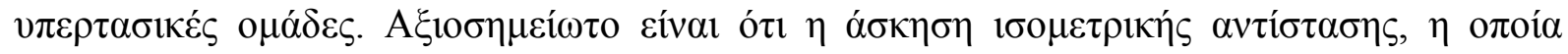

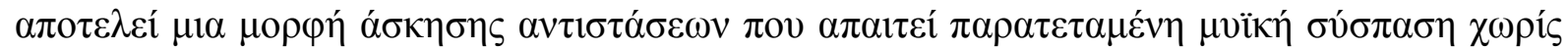

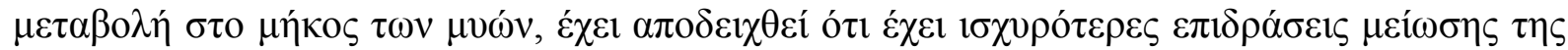

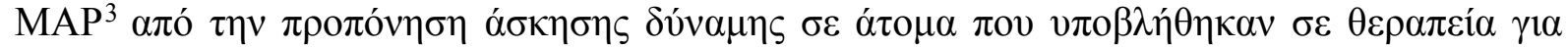

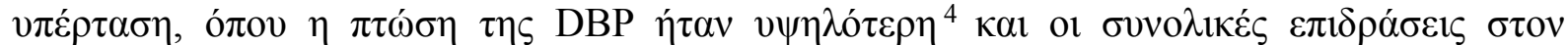

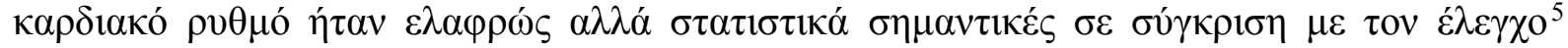
(Carlson et al. 2014).

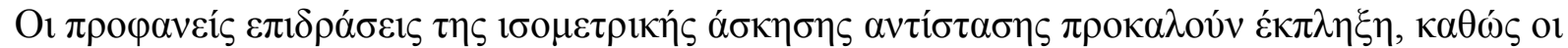

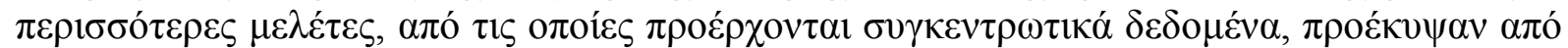

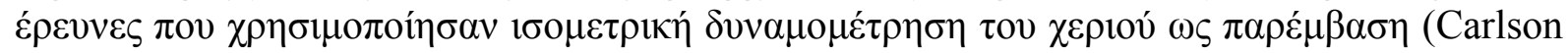

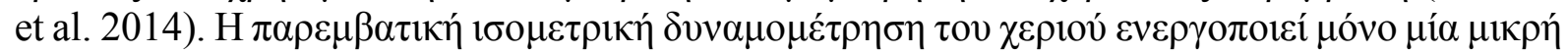
$\mu v$

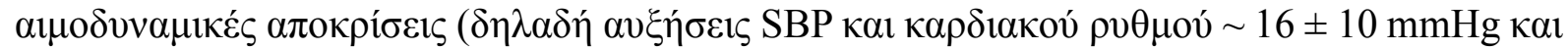

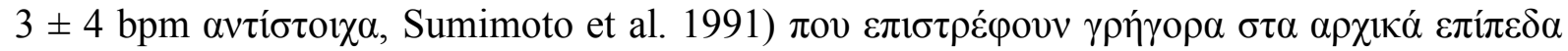

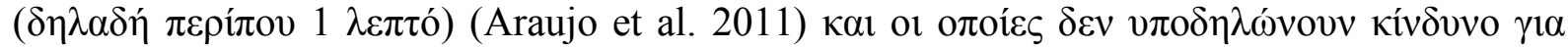

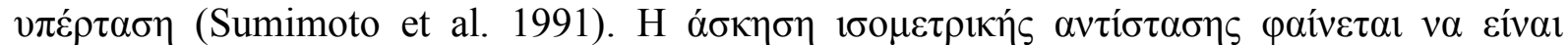

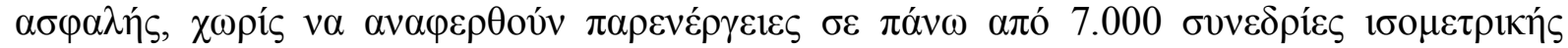

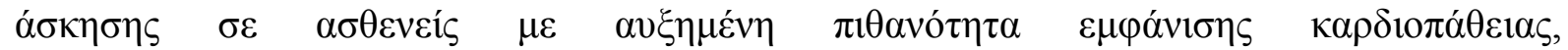

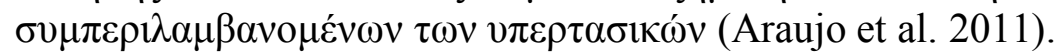

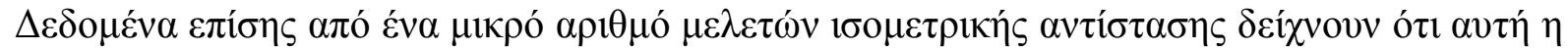

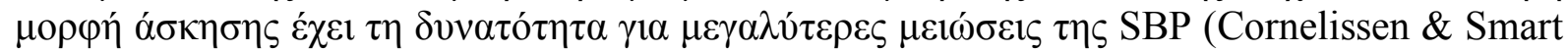

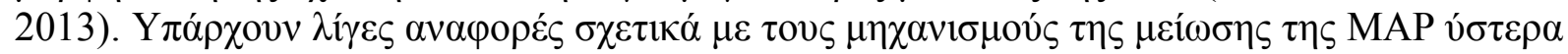

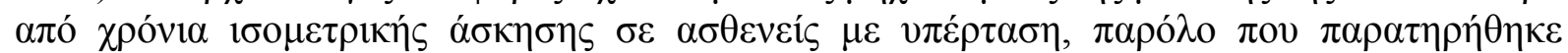

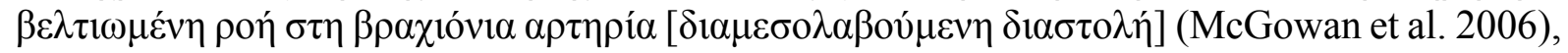

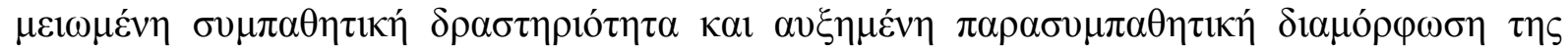

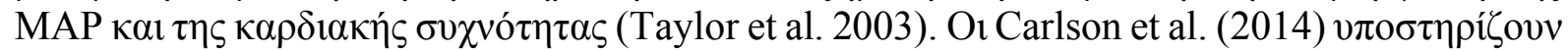

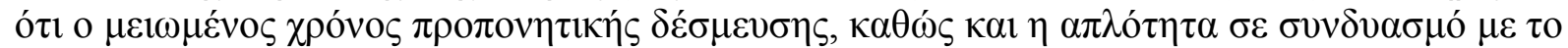

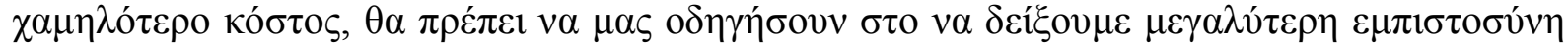

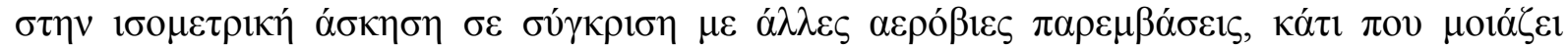

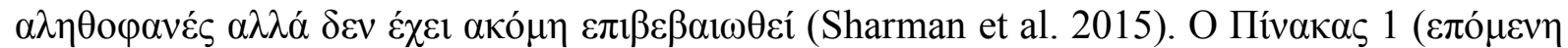

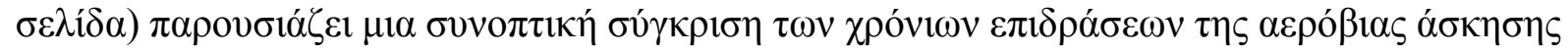

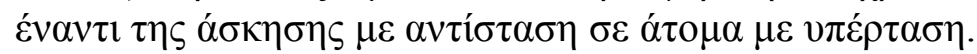

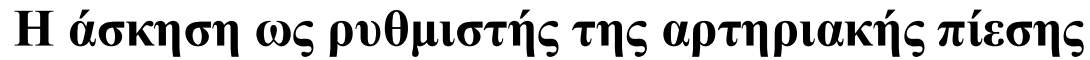

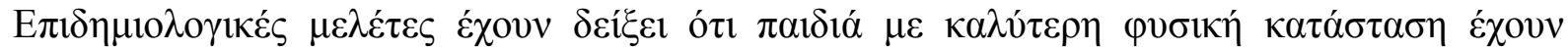

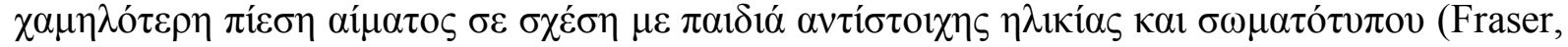

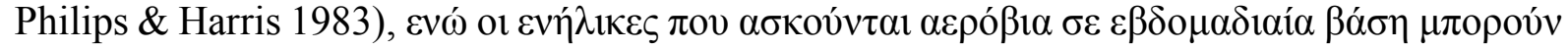

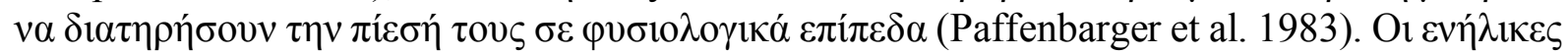

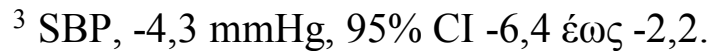

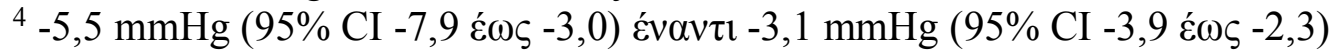

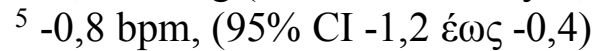




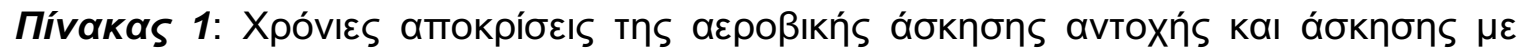

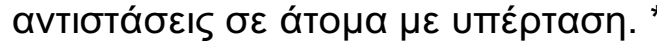

\begin{tabular}{|c|c|c|c|}
\hline 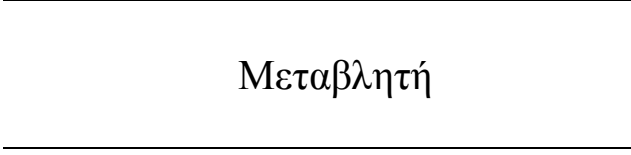 & 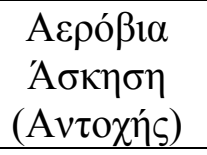 & 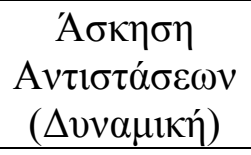 & 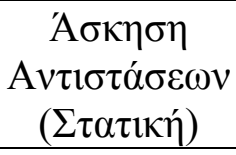 \\
\hline \multicolumn{4}{|l|}{ MAP } \\
\hline SBP & $\downarrow \downarrow \downarrow$ & $\leftrightarrow^{\dagger}$ & $\downarrow \downarrow \downarrow$ \\
\hline DBP & $\downarrow \downarrow \downarrow$ & $\downarrow$ & $\downarrow \downarrow \downarrow$ \\
\hline 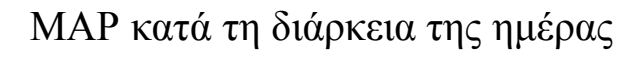 & $\downarrow \downarrow$ & -- & -- \\
\hline 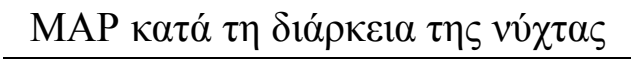 & $\leftrightarrow$ & -- & -- \\
\hline \multicolumn{4}{|l|}{$\Delta \varepsilon i ́ \kappa \tau \varepsilon \varsigma \pi \alpha \chi v \sigma \alpha \rho \kappa i ́ \alpha \varsigma$} \\
\hline BMI & $\downarrow \downarrow$ & -- & -- \\
\hline BW & $\downarrow \downarrow$ & $\leftrightarrow$ & -- \\
\hline $\mathrm{WC}$ & $\downarrow \downarrow$ & -- & -- \\
\hline$\% \mathrm{BF}$ & $\downarrow \downarrow$ & $\downarrow \downarrow$ & -- \\
\hline BG & $\downarrow \downarrow$ & -- & -- \\
\hline \multicolumn{4}{|l|}{$\Lambda \imath \pi i \delta \imath \alpha$} \\
\hline $\mathrm{CHOL}_{\text {tot }}$ & $\leftrightarrow$ & -- & -- \\
\hline LDL & $\leftrightarrow$ & -- & -- \\
\hline HDL & $\uparrow \uparrow$ & -- & -- \\
\hline TG & $\downarrow-$ & -- & -- \\
\hline HR & $\downarrow \downarrow$ & $\leftrightarrow$ & $\downarrow$ \\
\hline
\end{tabular}

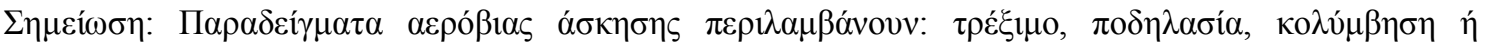

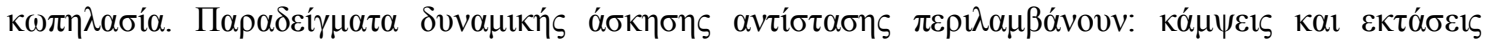

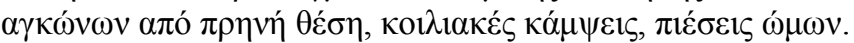

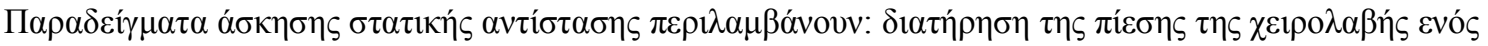

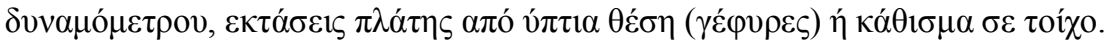

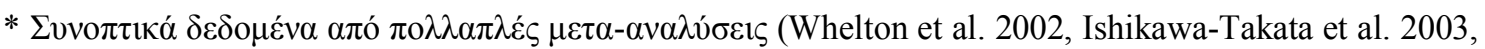

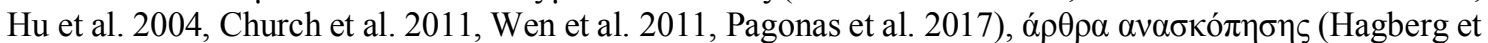
al. 2000, ACSM. 2004, Cornelissen \& Fagard 2005, Cornelissen et al. 2005, Fagard et al. 2007, Cornelissen

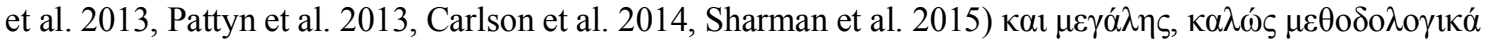

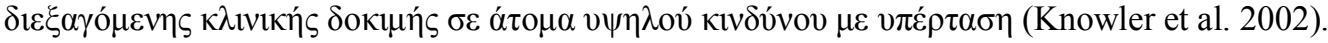

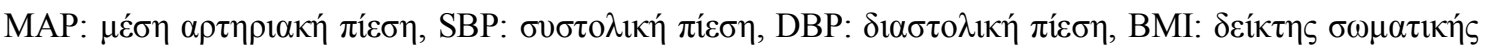

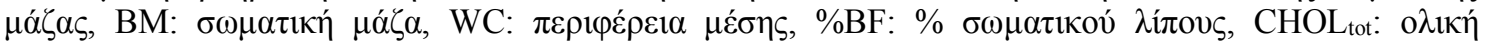

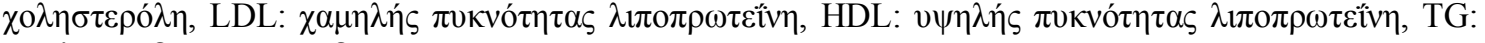

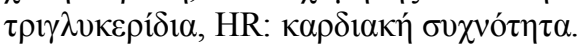

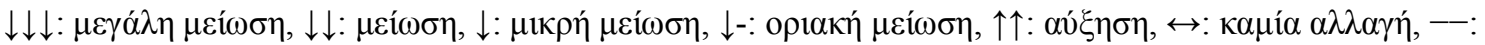

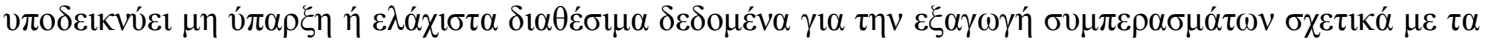

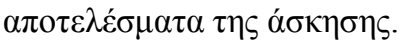

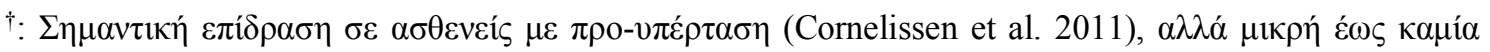

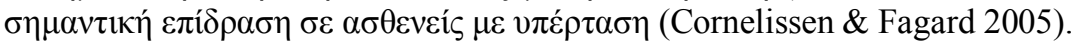




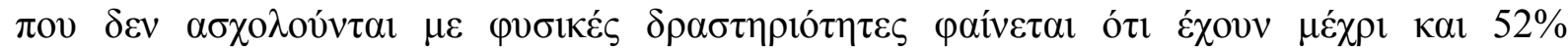

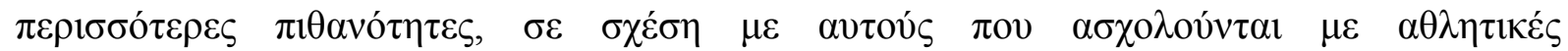

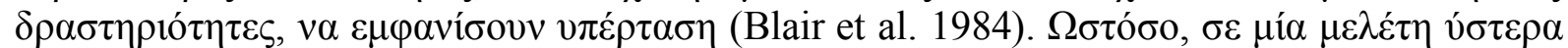

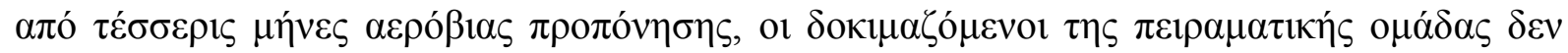

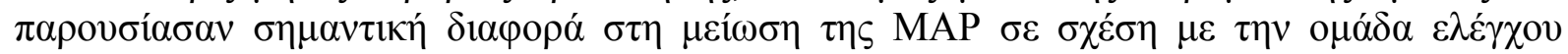

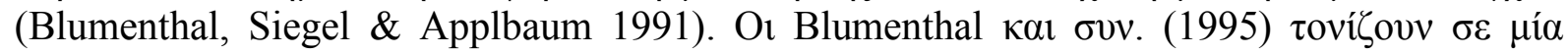

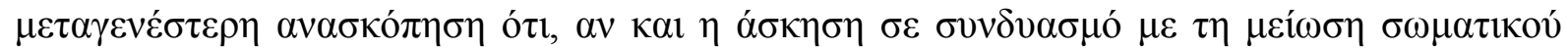

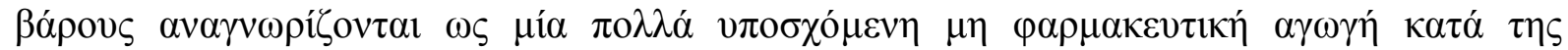

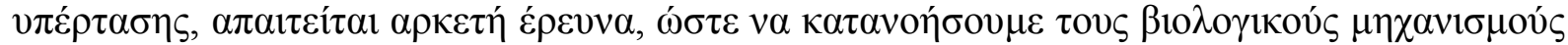

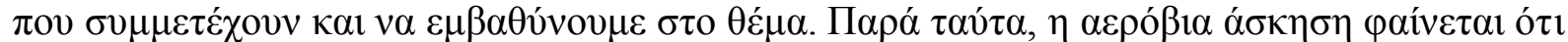

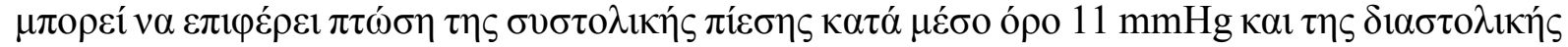

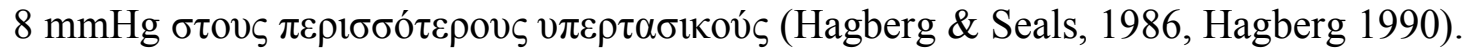

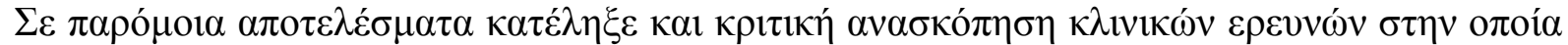

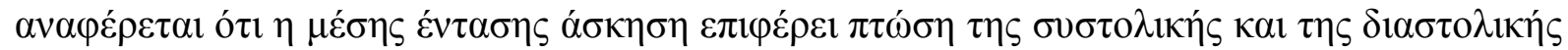

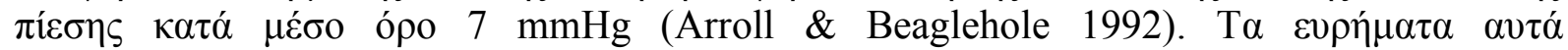

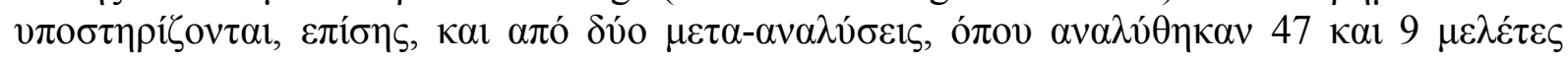

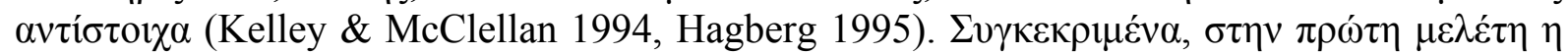

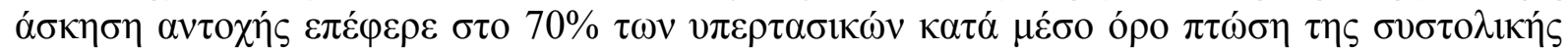

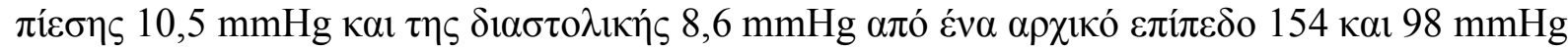

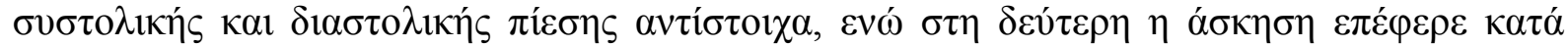

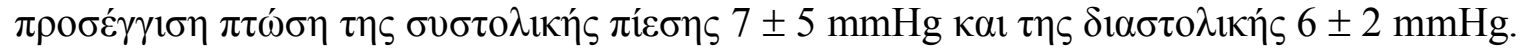

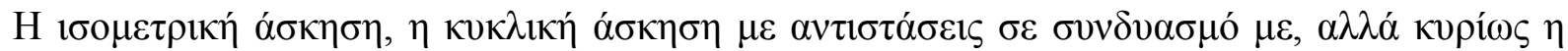

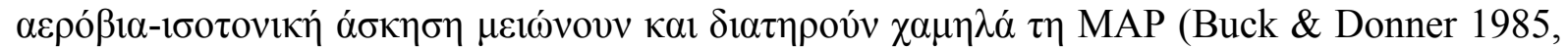
Hagberg, Ehsani, Goldring, Hernandez, Sinacore \& Holloszy 1984, Kelemen, Effron, Valenti,

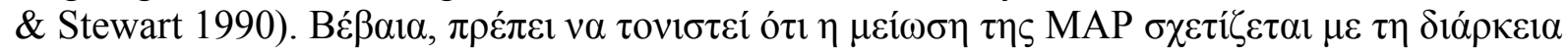

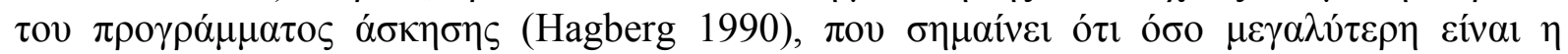

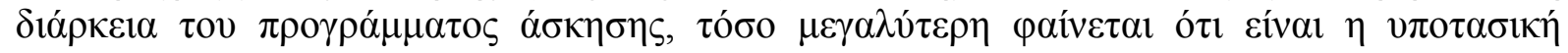

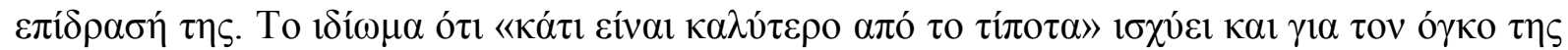

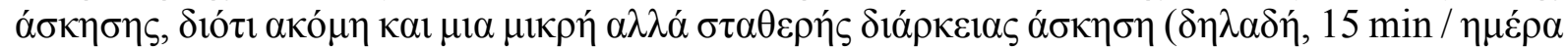

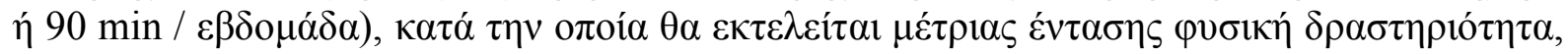

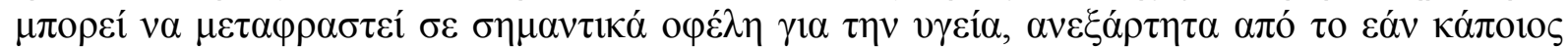

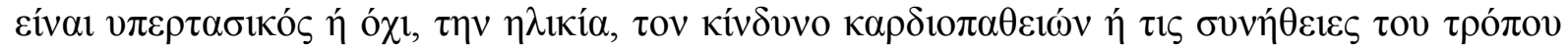

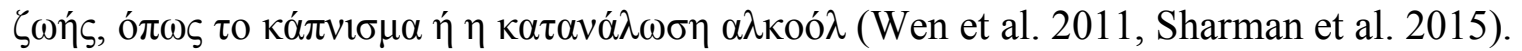

\section{$\Sigma v \mu \pi \varepsilon ́ \rho \alpha \sigma \mu \alpha$}

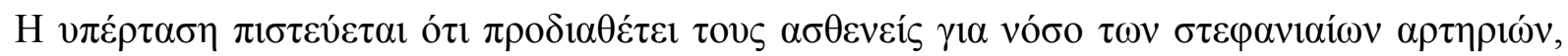

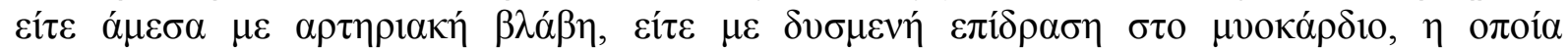

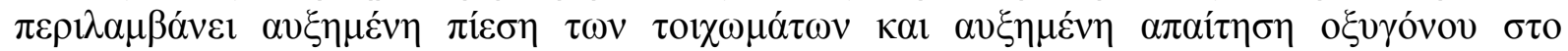

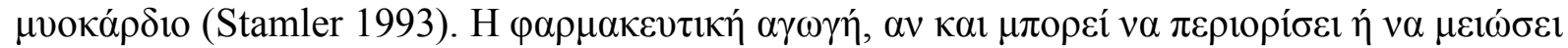

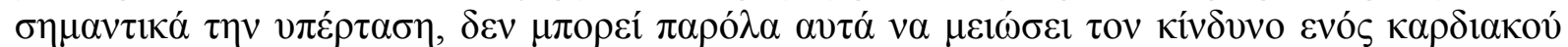

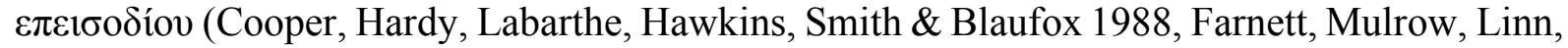

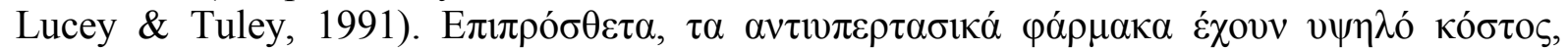

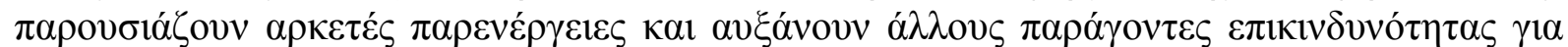

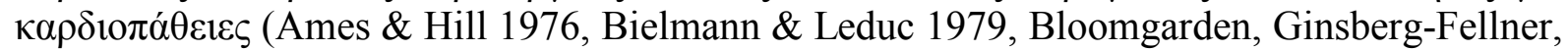

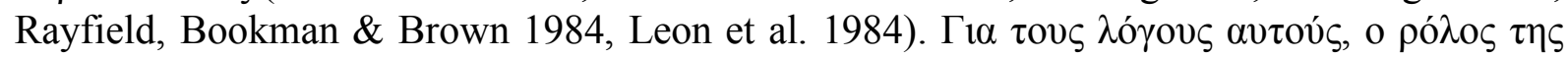

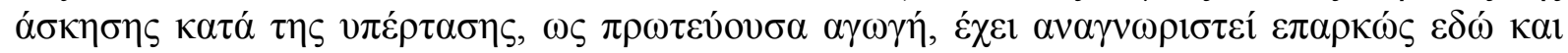

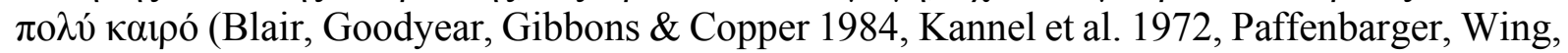

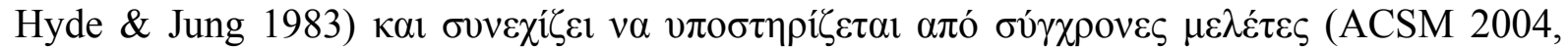


Hackam et al. 2010, Pescatello et al. 2015, Sharman et al. 2015, Hansen et al. 2017, Pagonas et al. 2017).

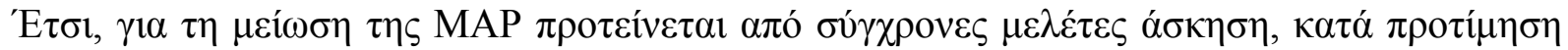

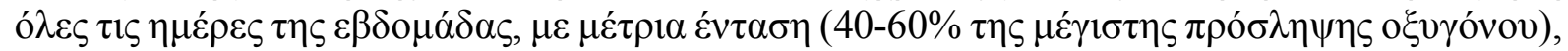

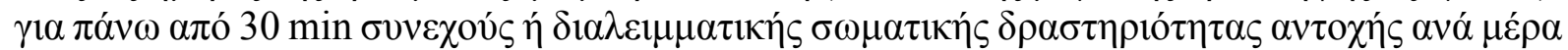
(ACSM 2004, Sharman \& Stowasser 2009, Hackam et al. 2010, Pagonas et al. 2017),

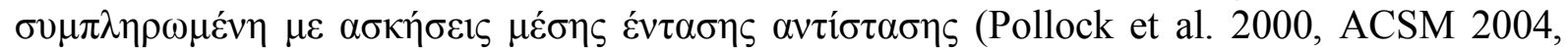

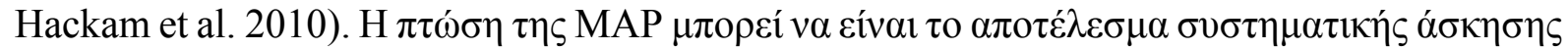

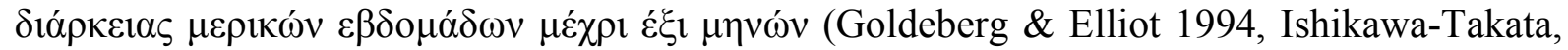

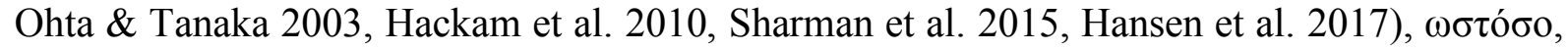

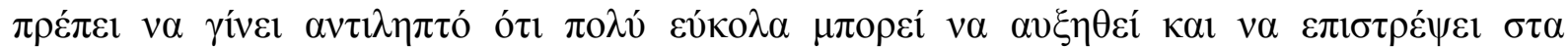

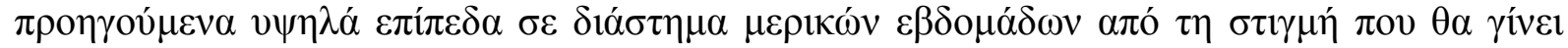

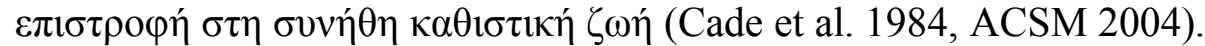

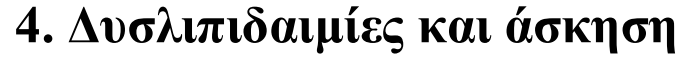

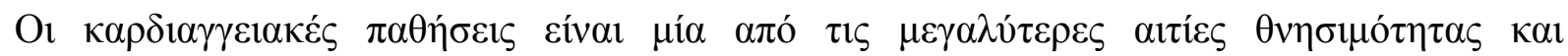

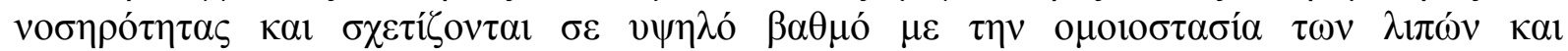

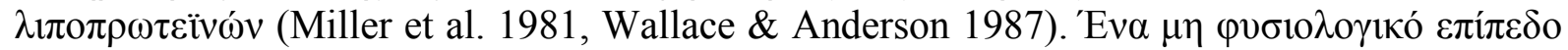

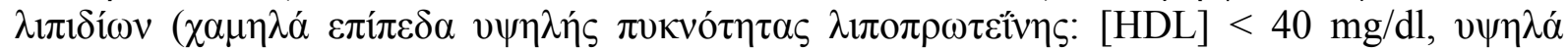

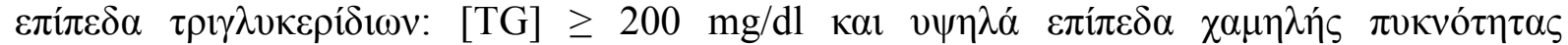

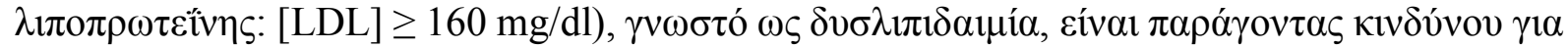

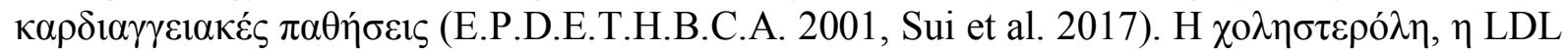

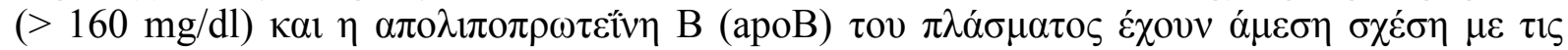

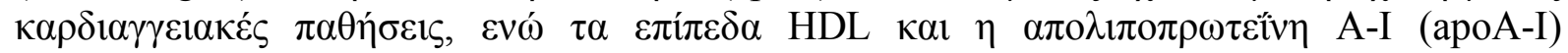

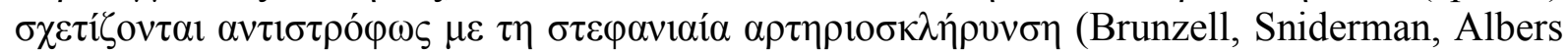

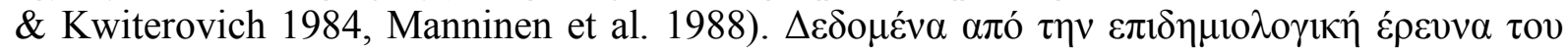

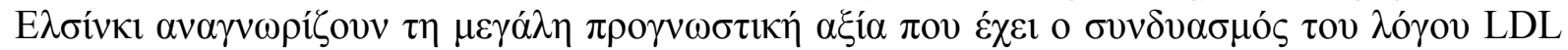

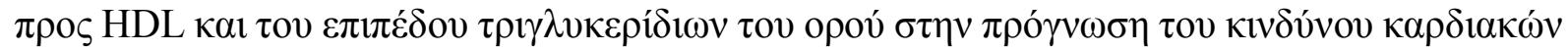

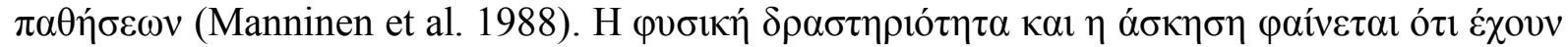

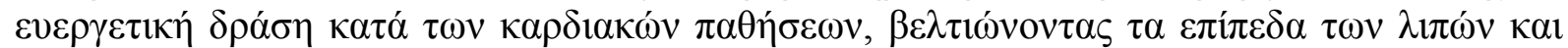

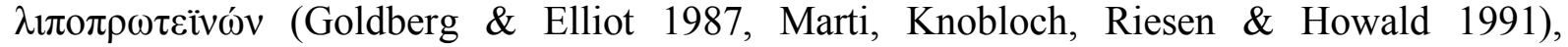

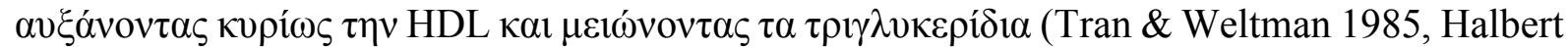
et al. 1999, Leon \& Sanchez 2001, Carroll \& Dudfield 2004, Duncan et al. 2005). Me $\alpha \lambda \lambda \alpha$

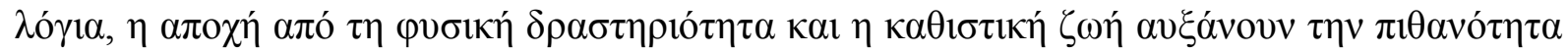

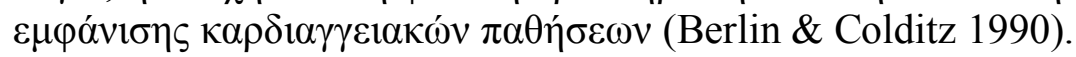

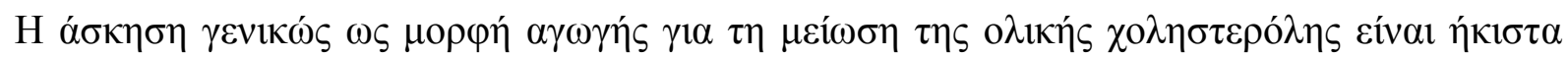

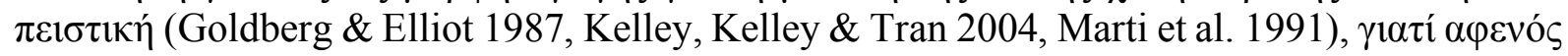

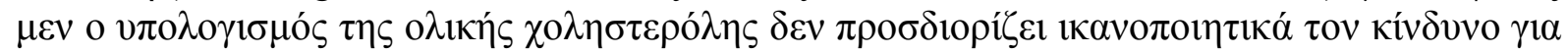

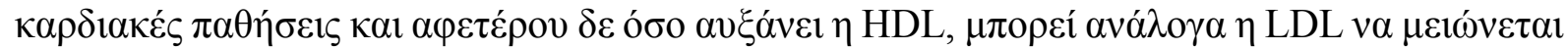

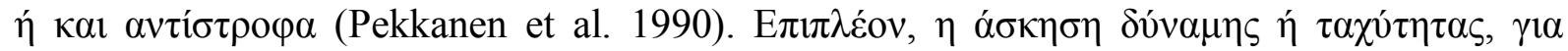

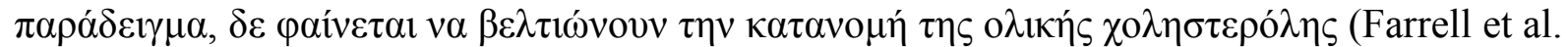

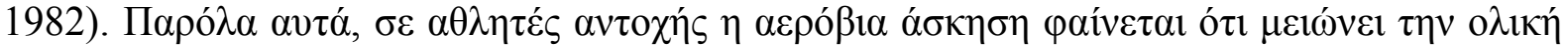

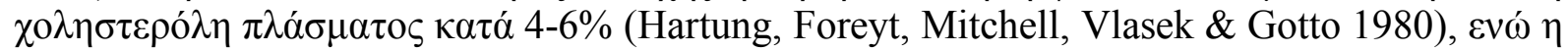

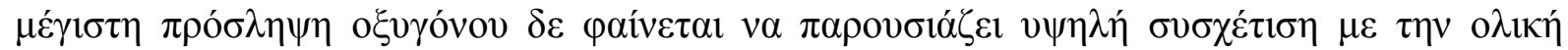

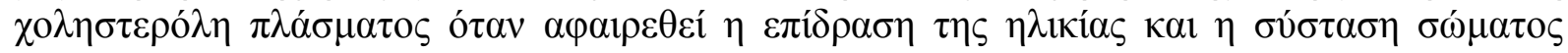

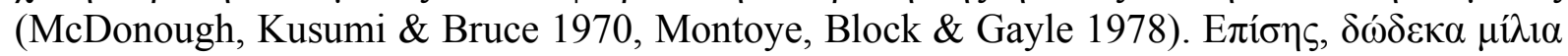

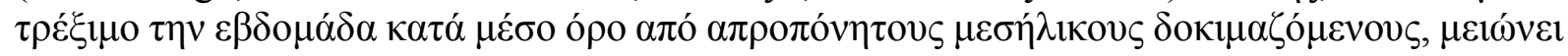

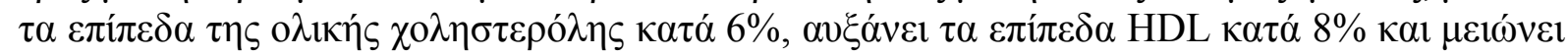

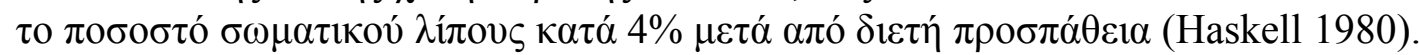




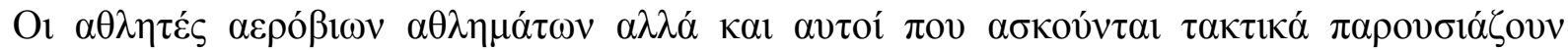

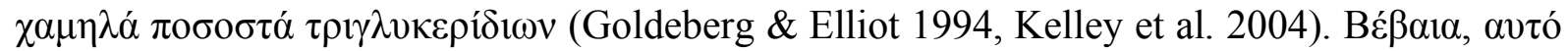

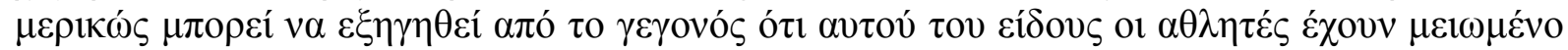

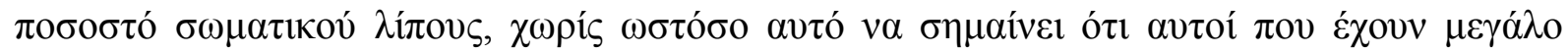

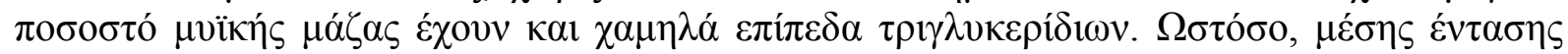

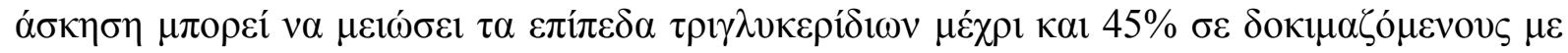

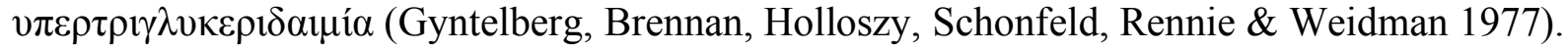

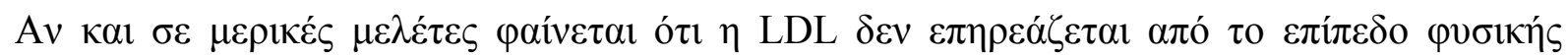

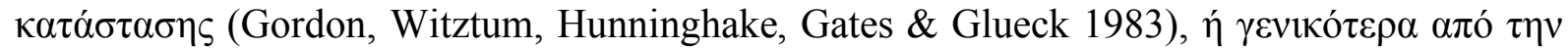

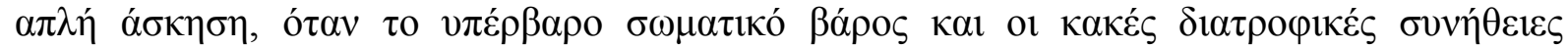

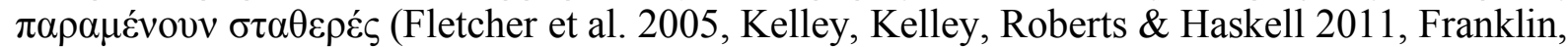

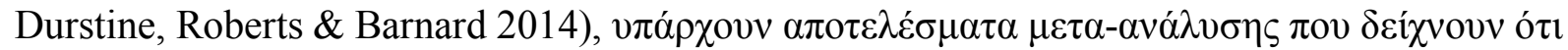

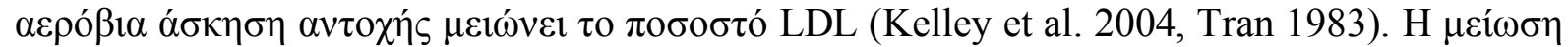

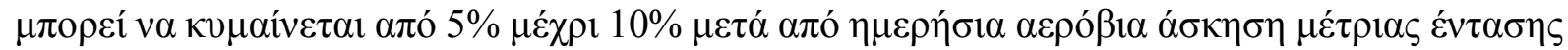

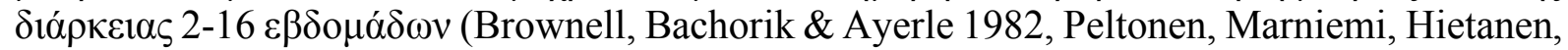

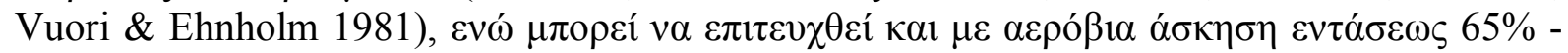

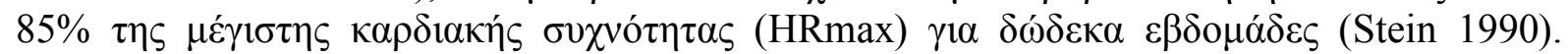

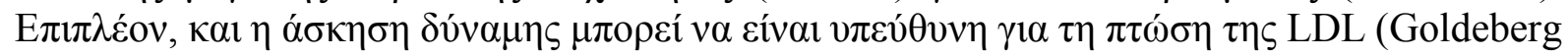
et al 1984).

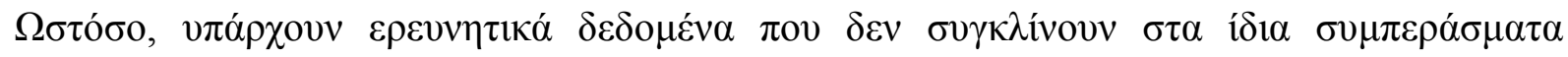
(Kokkinos 1988, Manning 1991, Williams, Wood, Haskell \& Vranizan 1982). T $\alpha$

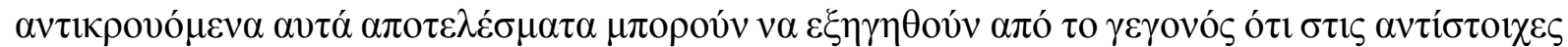

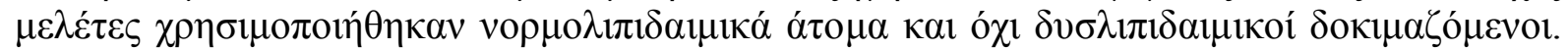

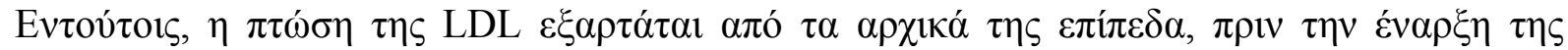

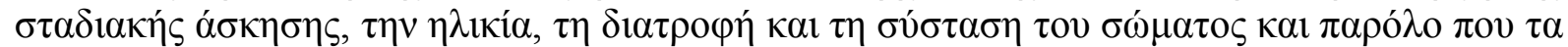

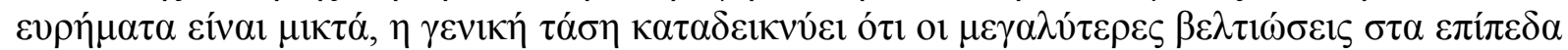

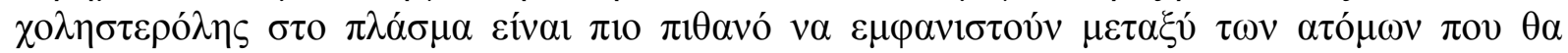

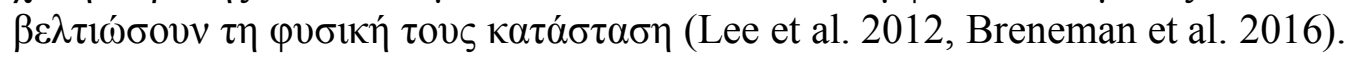

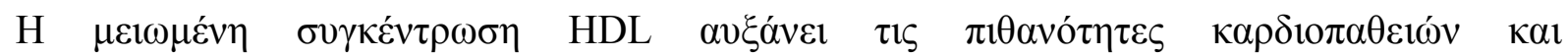

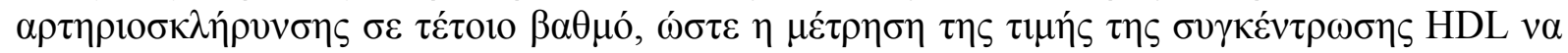

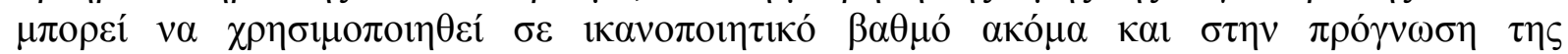

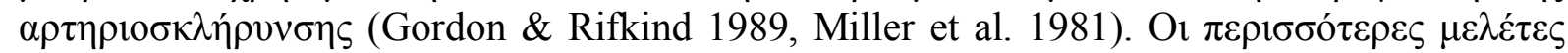

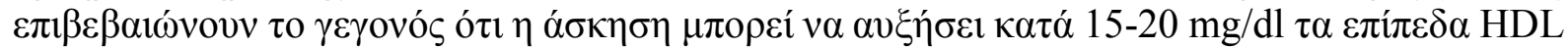

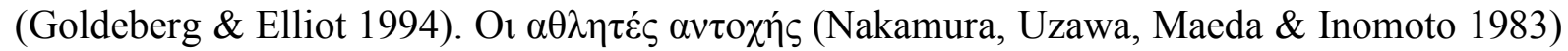

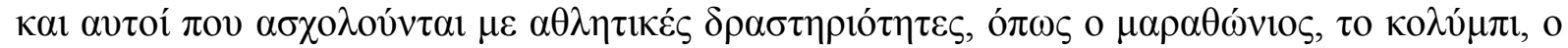

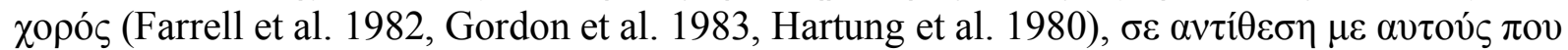

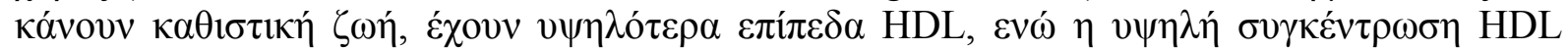

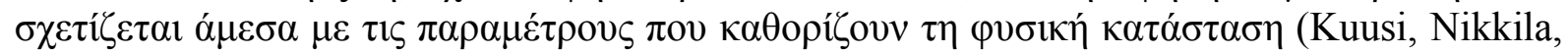

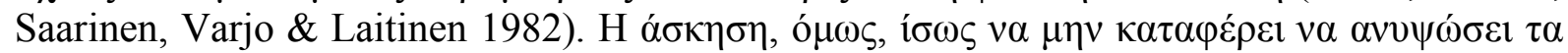

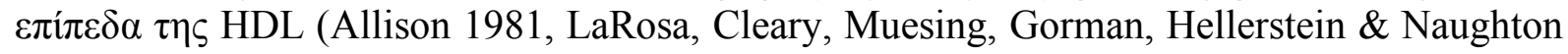
1982, Lipson, Bonow, Schaefer, Brewer \& Lindgren 1980, Savage, Petratis, Thomson, Berg,

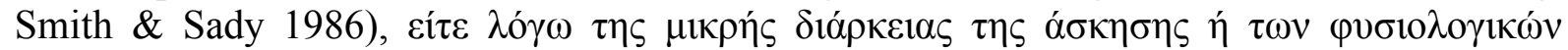

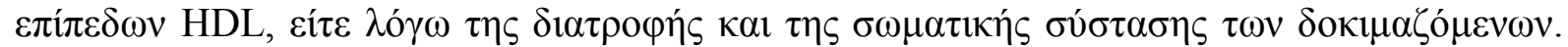

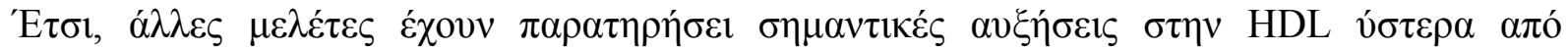

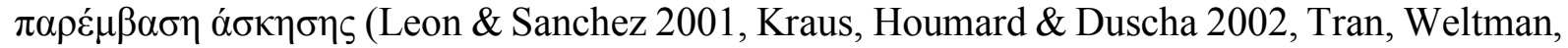

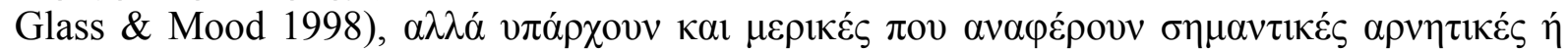

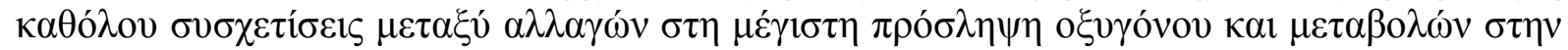

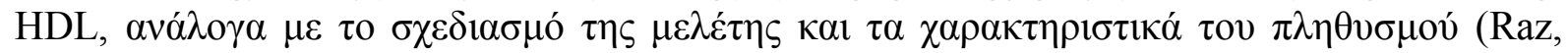

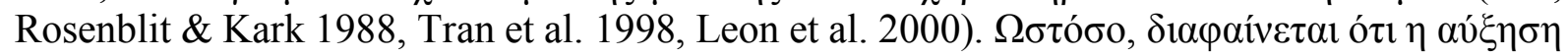




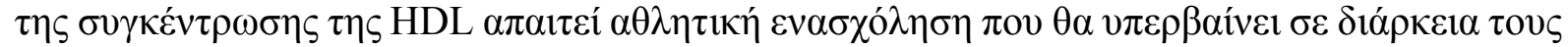

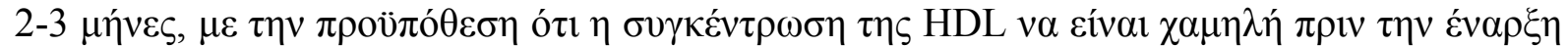

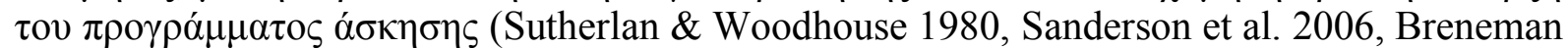
et al. 2016).

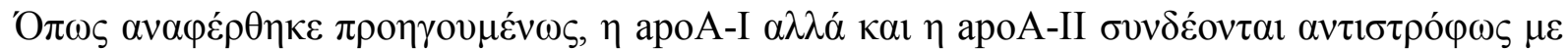

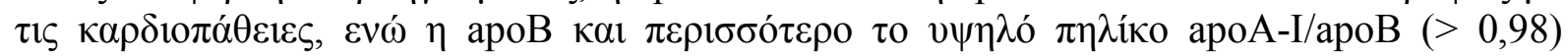

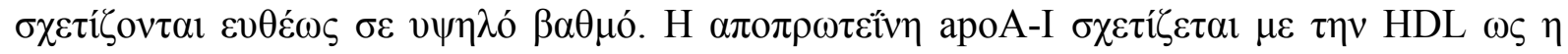

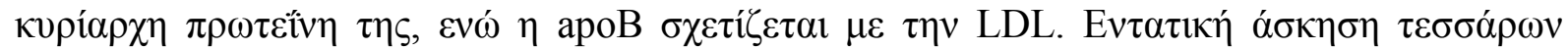

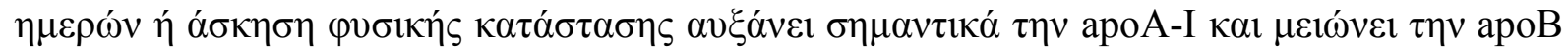
(Danner, Wieling, Havekes, Leuven, Smit \& Dunning 1984, Gordon et al. 1983, Magnus, Borresen, Opstad, Bugge \& Berg 1984, Wood, Stefanick, Williams \& Haskell 1991).

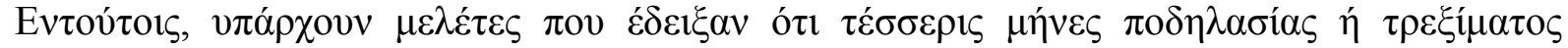

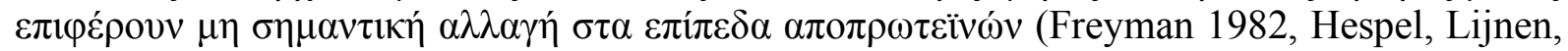

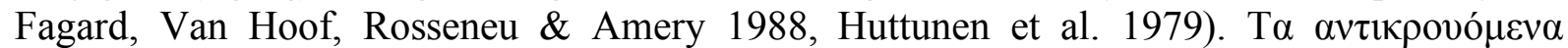

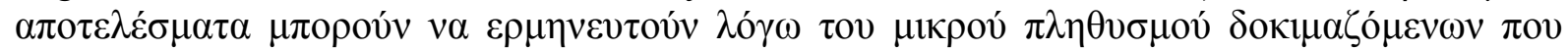

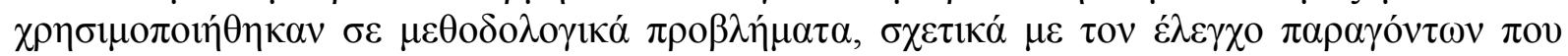

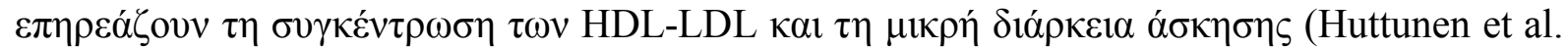

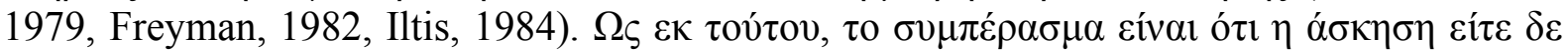

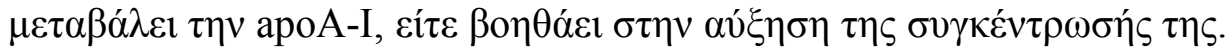

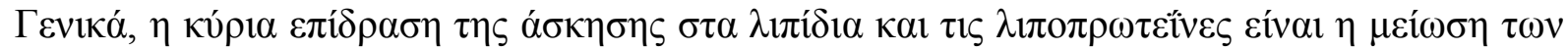

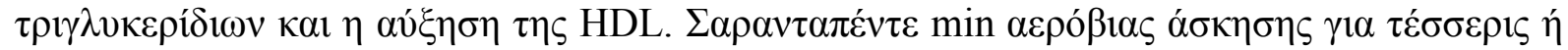

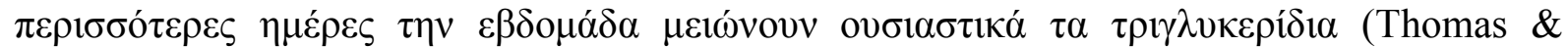

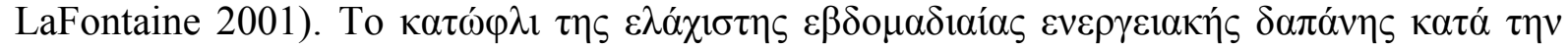

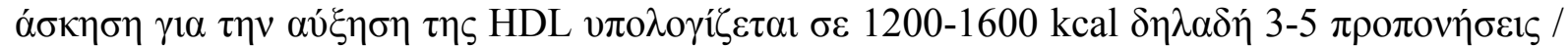

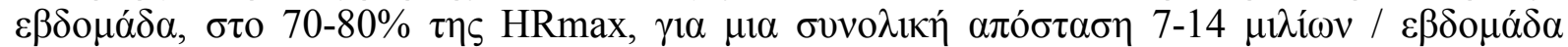

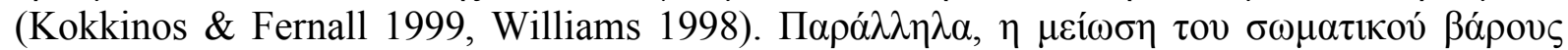

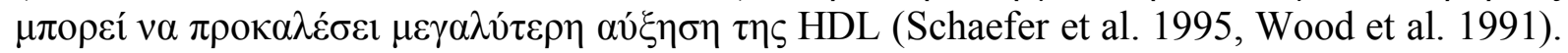

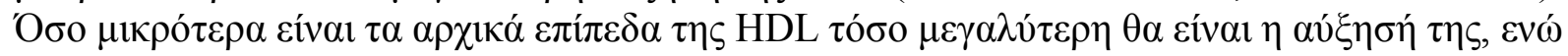

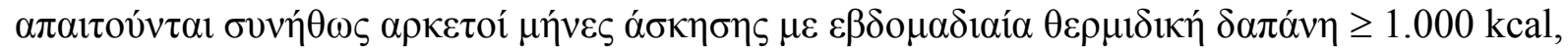

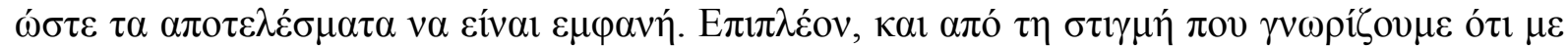

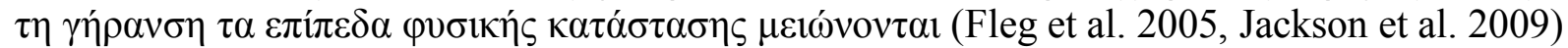

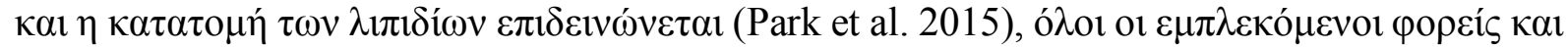

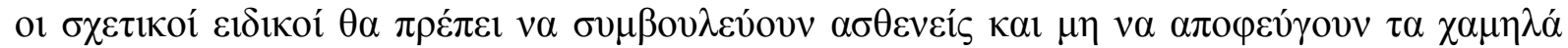

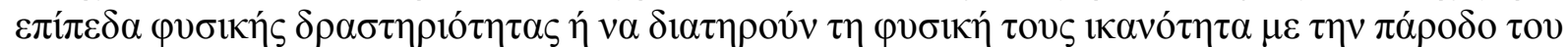

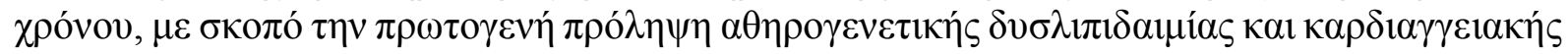
vóбov.

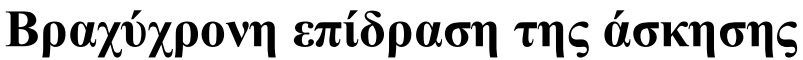

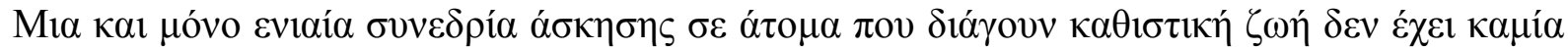

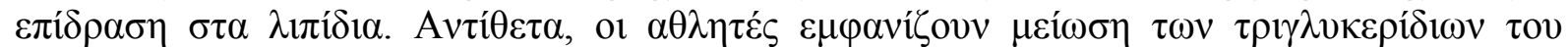

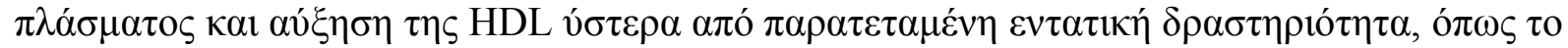

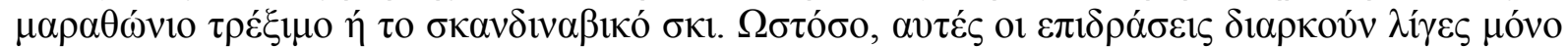

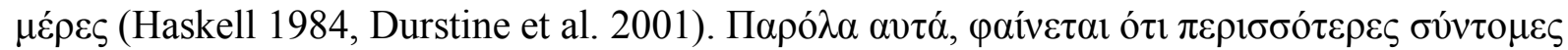

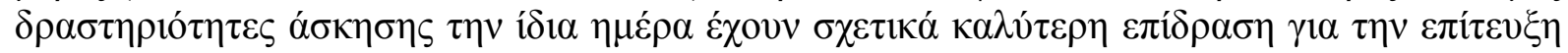

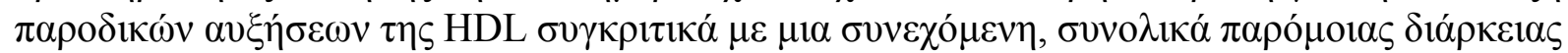

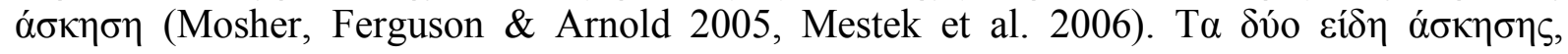

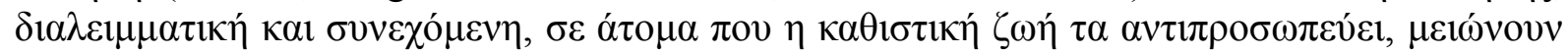

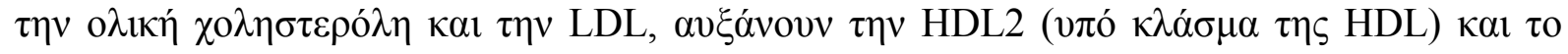




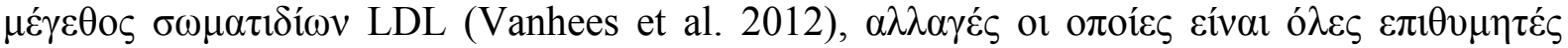
(Altena et al. 2006).

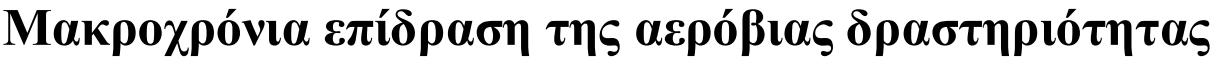

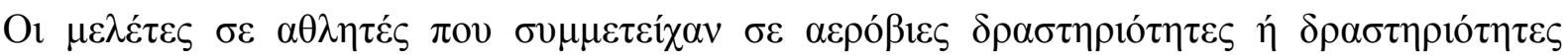

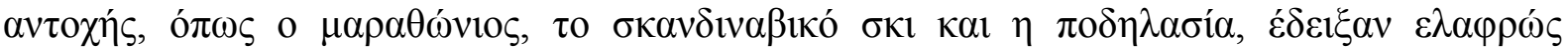

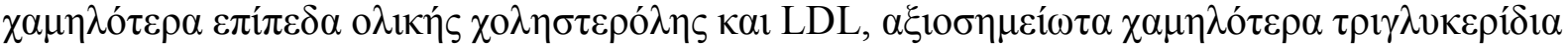

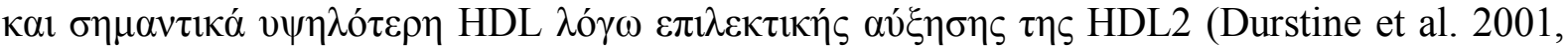

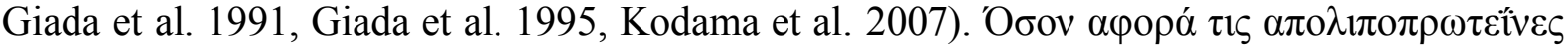

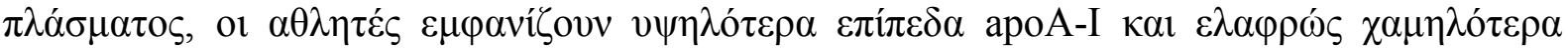

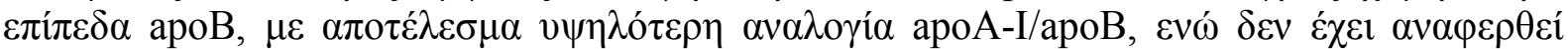

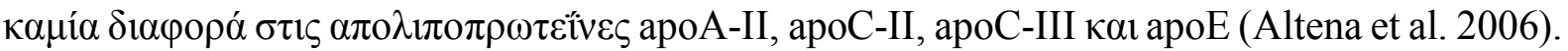

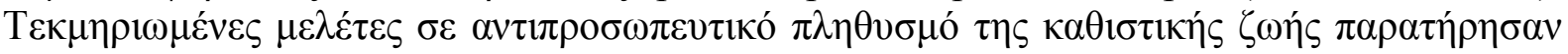

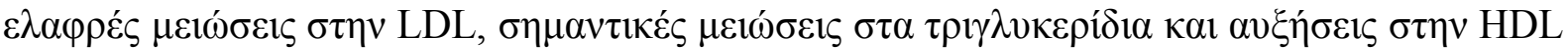

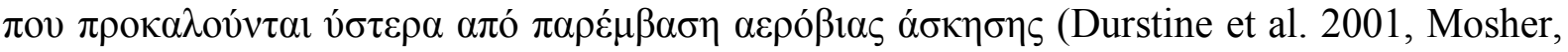
Ferguson \& Arnold 2005, Mestek et al. 2006, Altena et al. 2006, Giada et al. 1991, Giada et al.

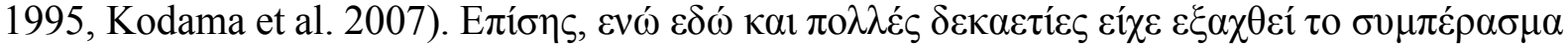

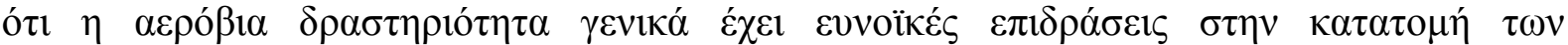

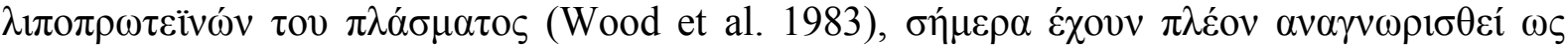

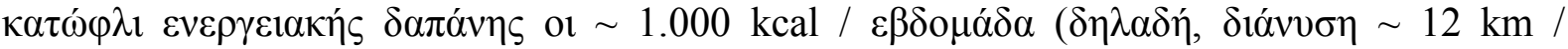

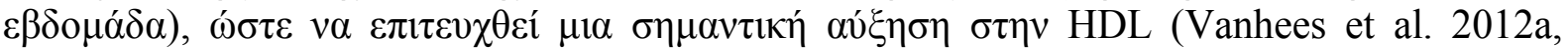
Vanhees et al. 2012b, Piepoli et al. 2016).

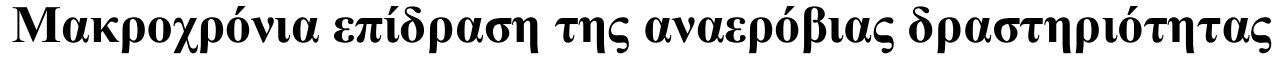

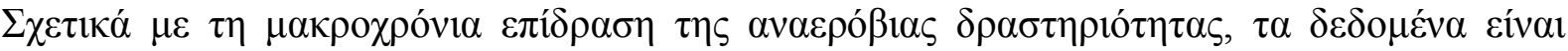

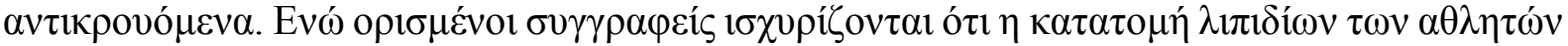

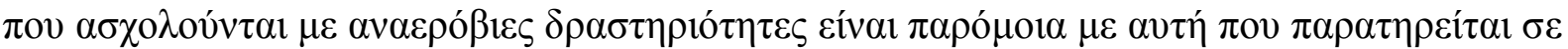

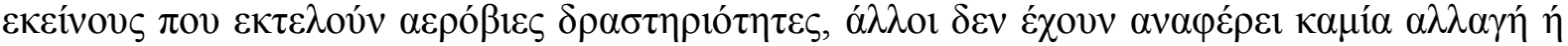

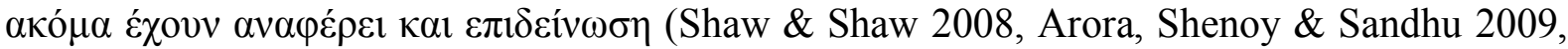
Marques et al. 2009).

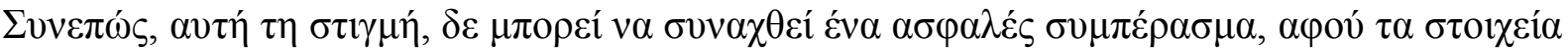

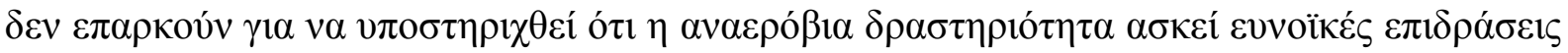

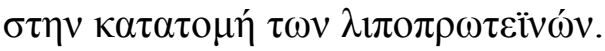

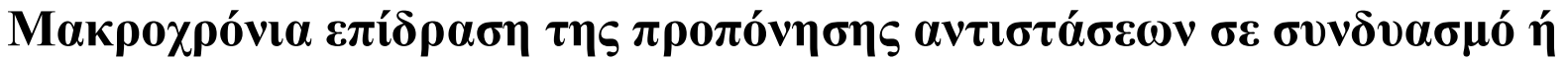 $\mu \eta \mu \varepsilon \alpha \varepsilon \rho o ́ \beta ı \alpha ~ \delta \rho \alpha \sigma \tau \eta \rho ı ́ \tau \eta \tau \alpha$}

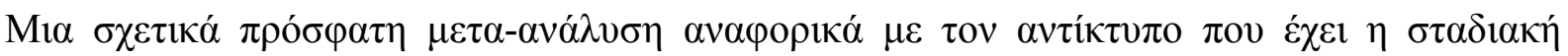

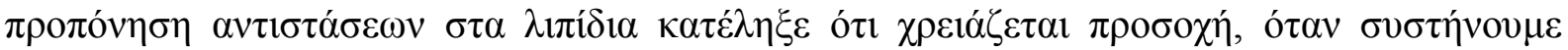

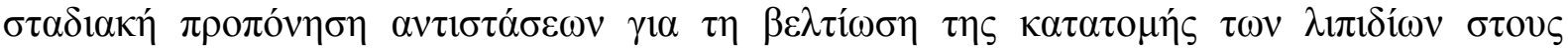

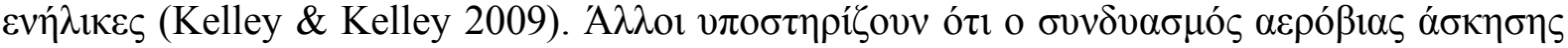

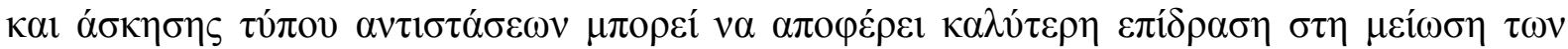

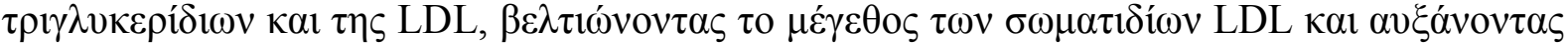

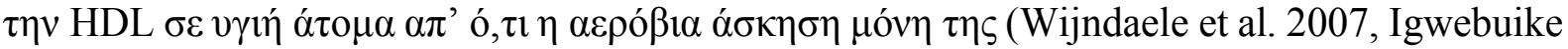
et al. 2008, Pitsavos et al. 2009, Tambalis et al. 2009).

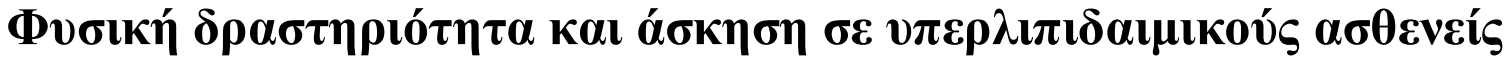




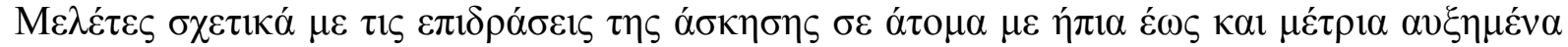

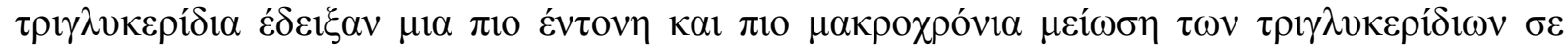

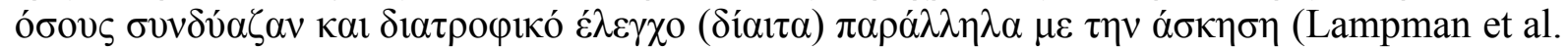

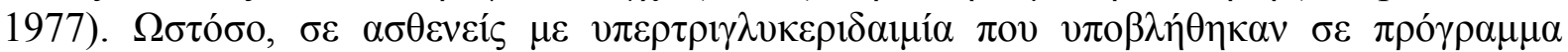

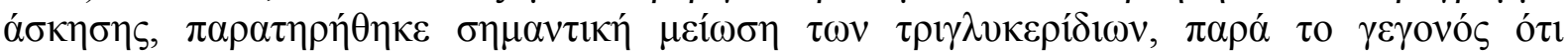

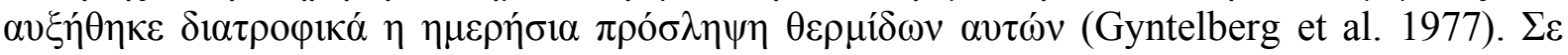

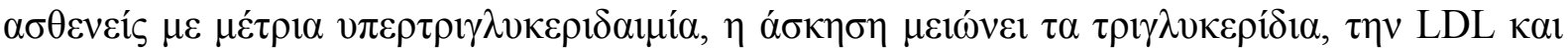

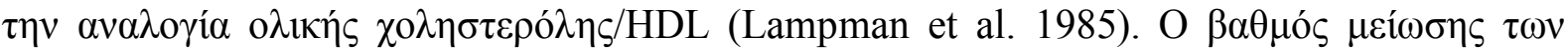

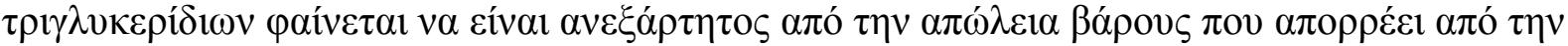

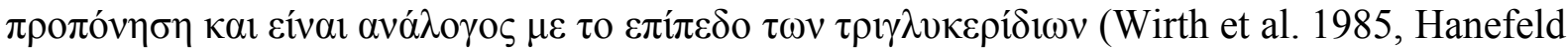

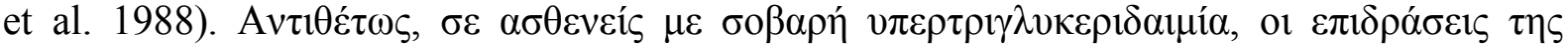

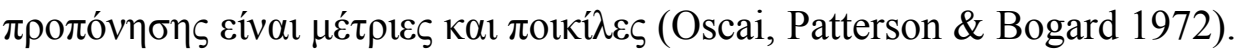

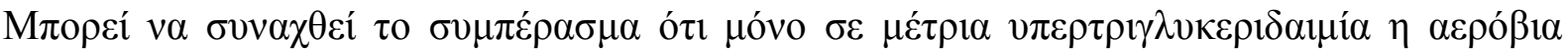

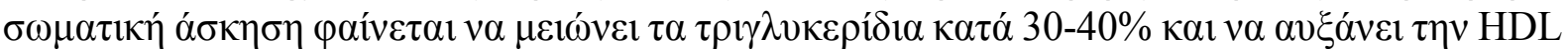

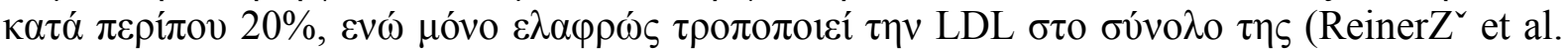

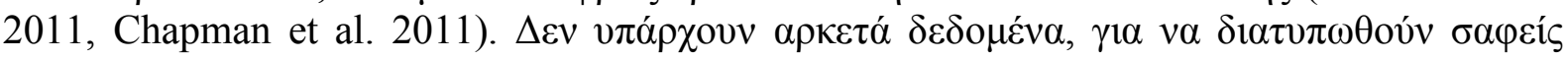

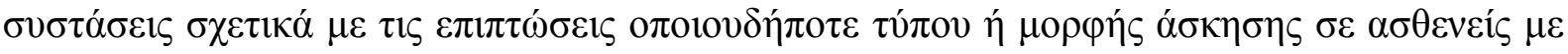

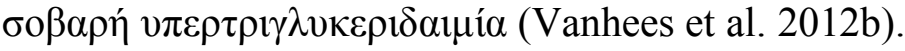

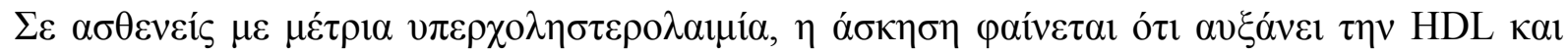

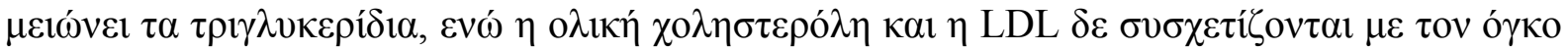

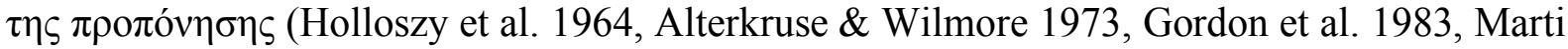

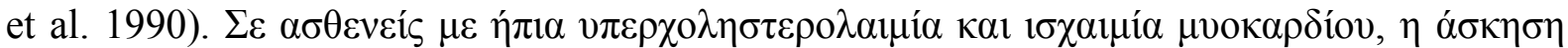

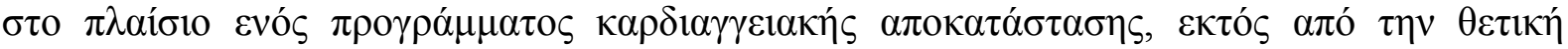

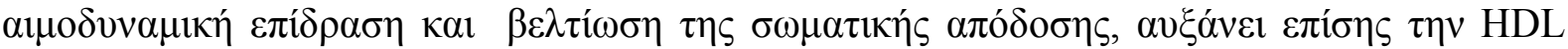

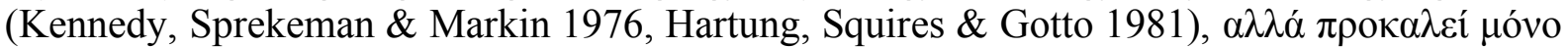

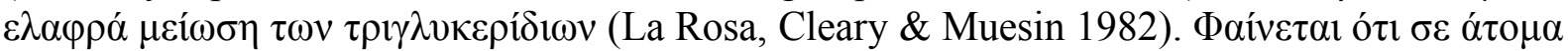

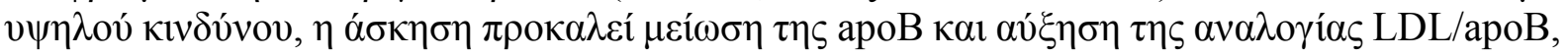

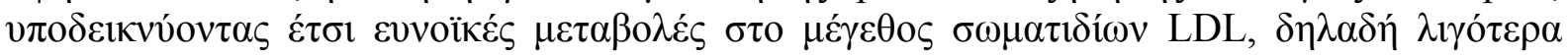

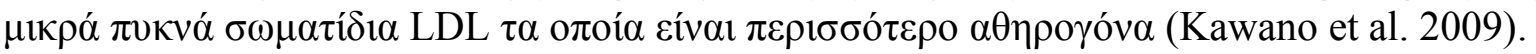

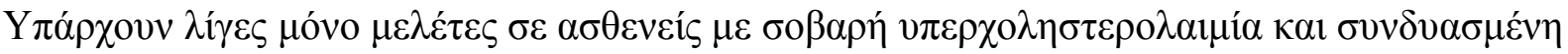

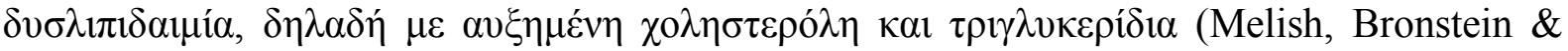

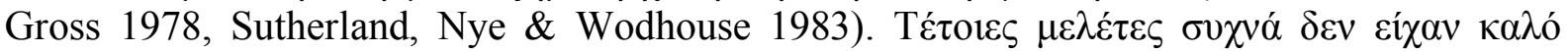

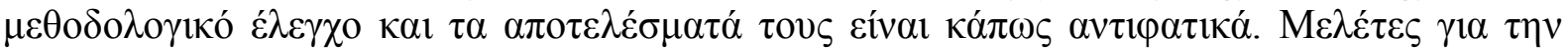

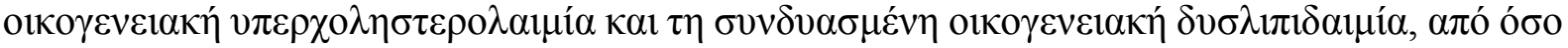

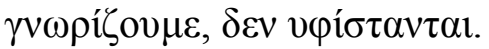

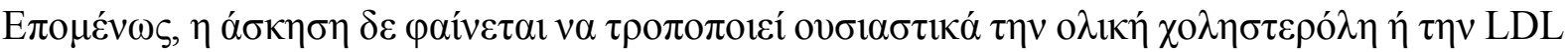

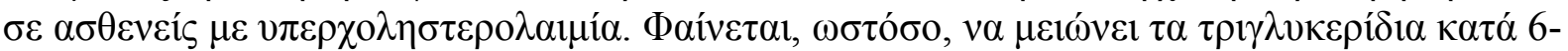

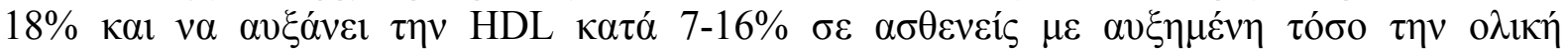

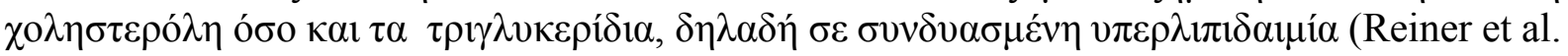
2011, Chapman et al. 2011).

\section{$\Sigma v \mu \pi \varepsilon ́ \rho \alpha \sigma \mu \alpha$}

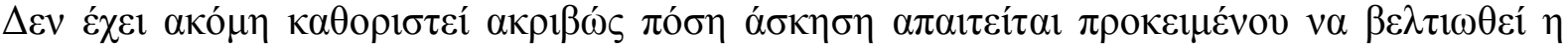

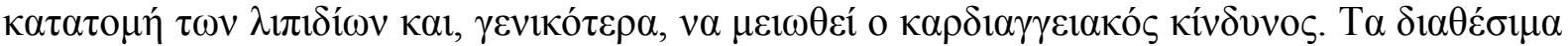

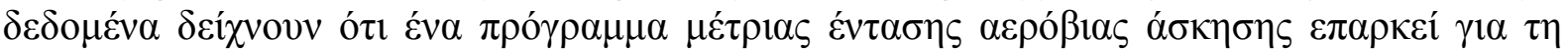

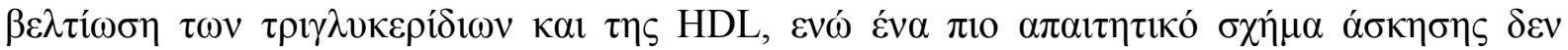

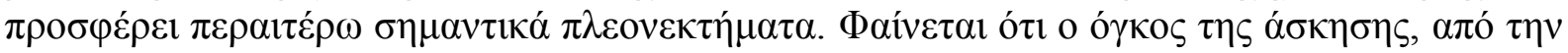

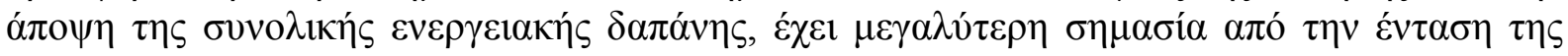




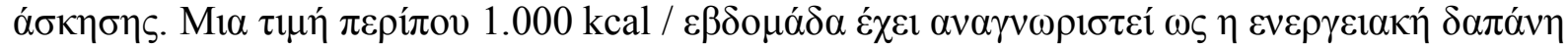

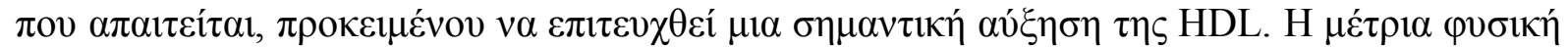

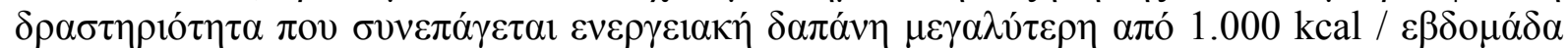

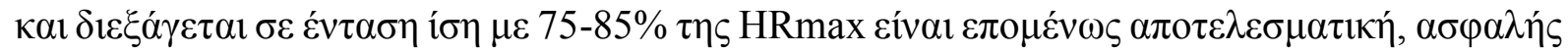

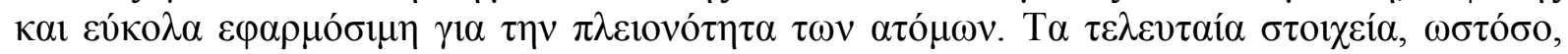

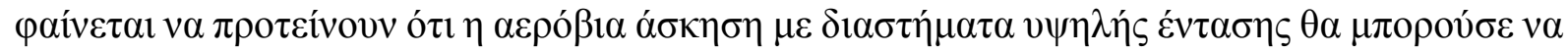

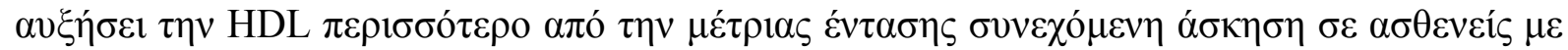

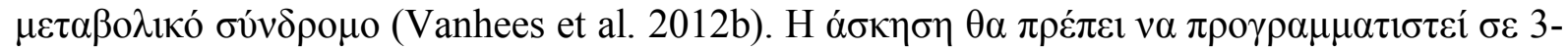

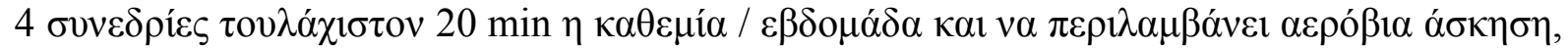

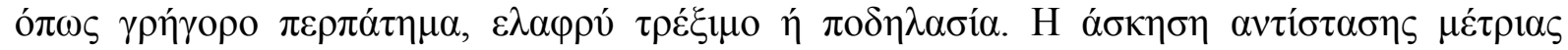

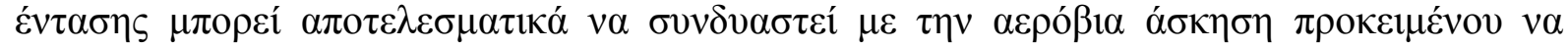

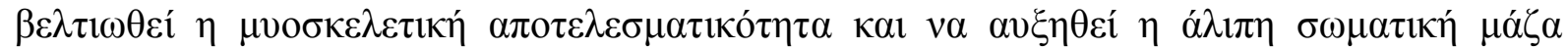
(Vanhees et al. 2012b).

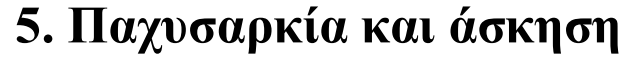

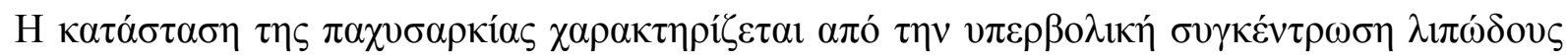

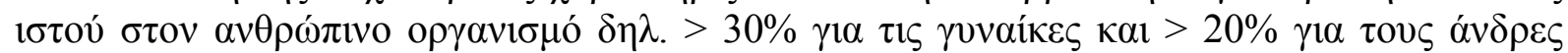

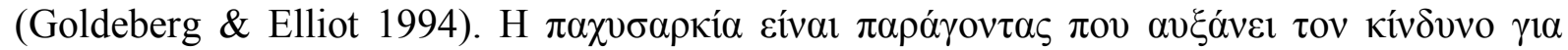

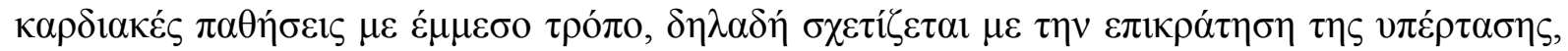

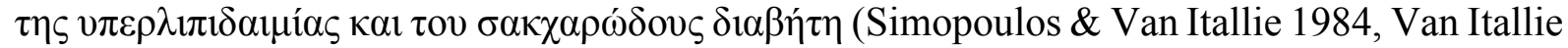

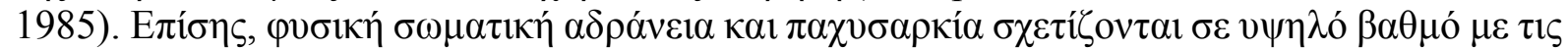

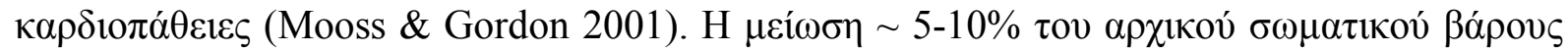

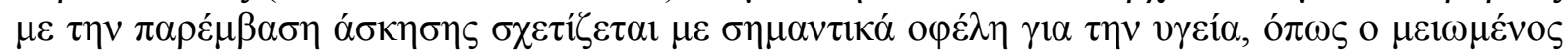

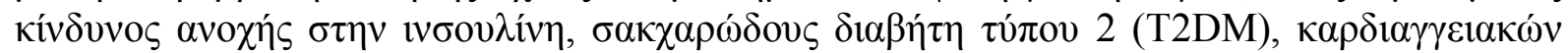

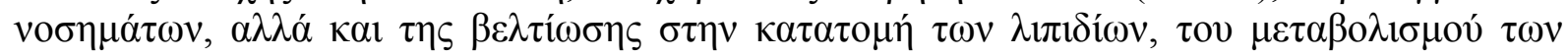

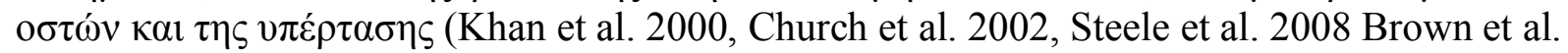

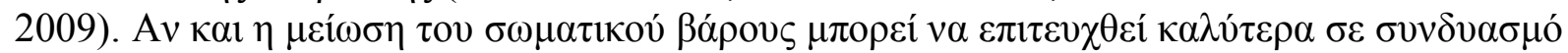

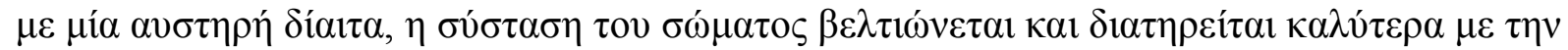
$\alpha ́ \sigma \kappa \eta \sigma \eta$ (Donnelly et al. 2004, Grilo \& Brownell 2001, Pavlou, Krey \& Steffee 1989). To

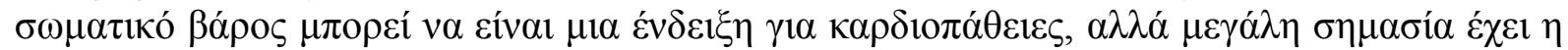

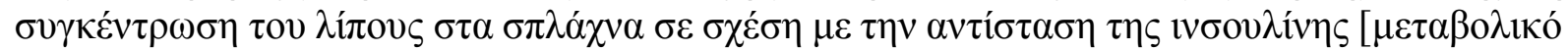

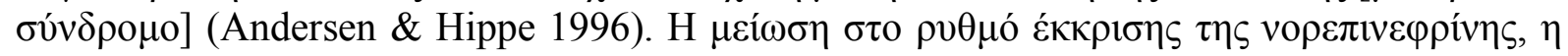

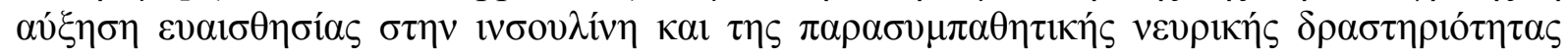

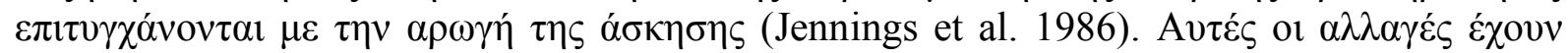

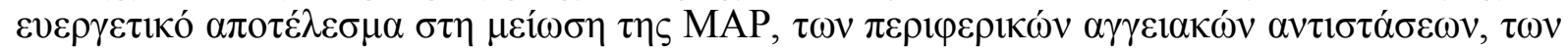

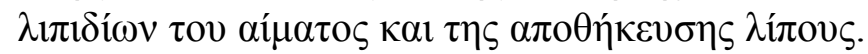

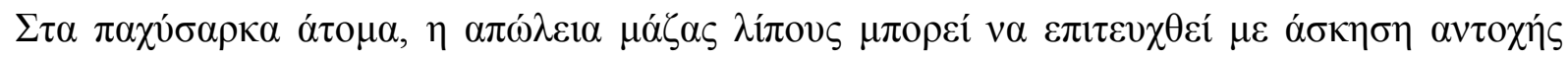
(Miller, Koceja \& Hamilton 1997, Khan et al. 2000, Church et al. 2002, Steele et al. 2008,

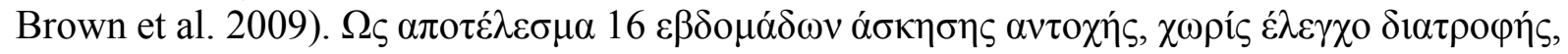

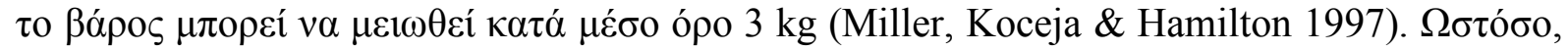

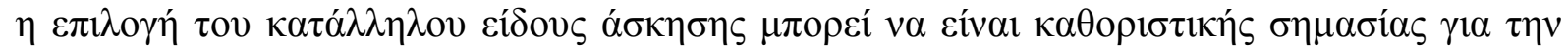

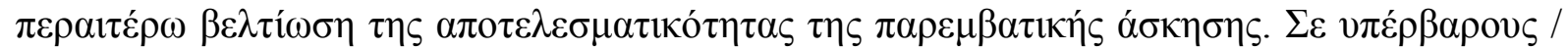

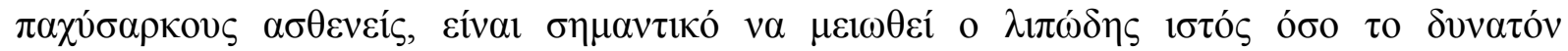

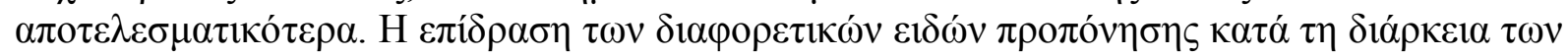

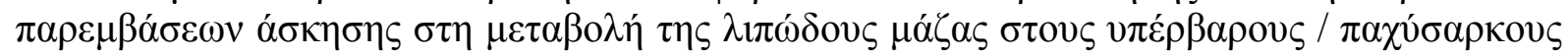

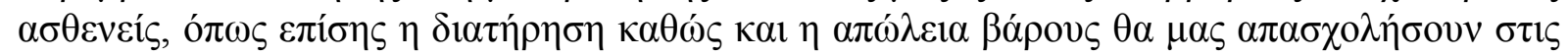

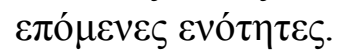

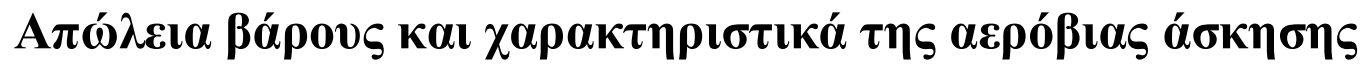




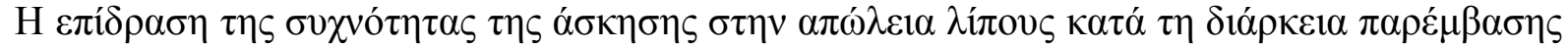

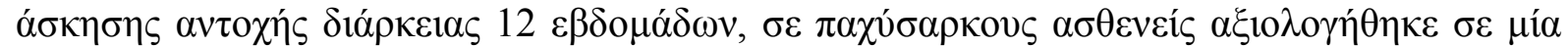

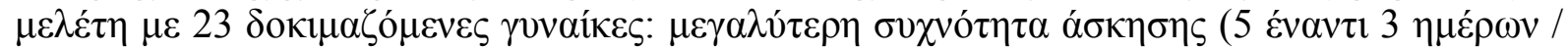

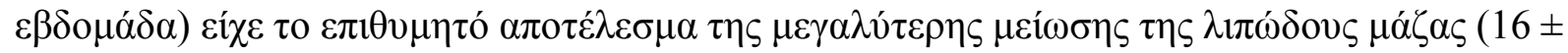

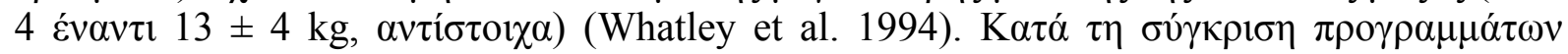

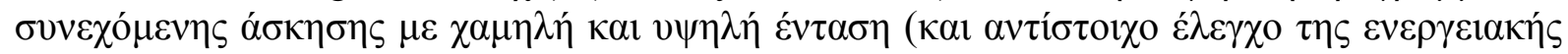

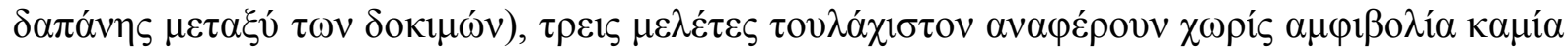

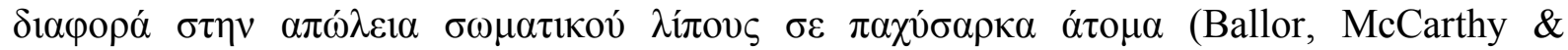
Wilterdink 1994, Leutholtz et al. 1995, van Aggel-Leijssen et al. 2002). $\Sigma \varepsilon \mu 1 \alpha \alpha \dot{\alpha} \lambda \lambda \eta \mu \varepsilon \lambda \varepsilon ́ \tau \eta$

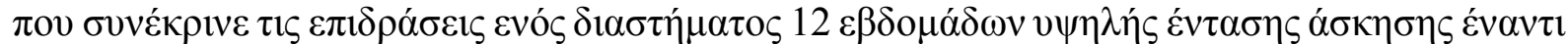

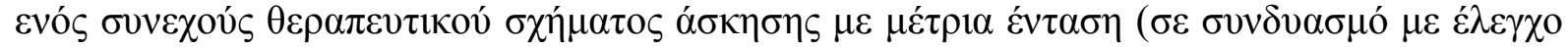

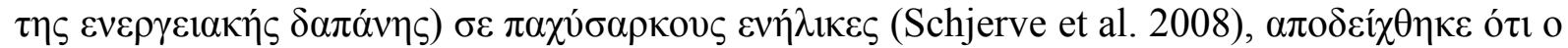

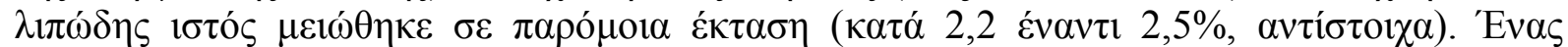

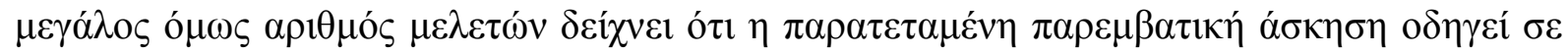

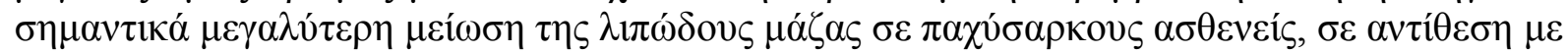
$\tau \eta \beta \rho \alpha \chi \varepsilon i ́ \alpha \pi \alpha \rho \varepsilon ́ \mu \beta \alpha \sigma \eta \alpha ́ \alpha \kappa \eta \sigma \eta \varsigma$ (Hansen, Dendale, van Loon LJ \& Meeusen 2010). O1 $\mu \varepsilon \lambda \varepsilon ́ \tau \varepsilon \varsigma$

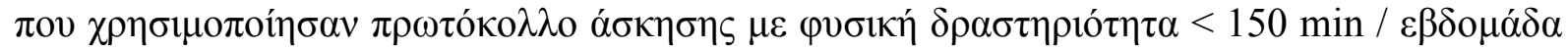

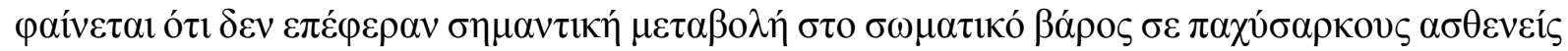

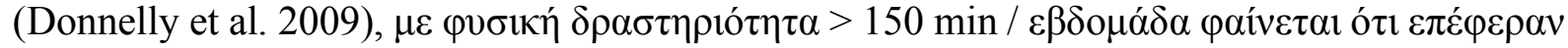

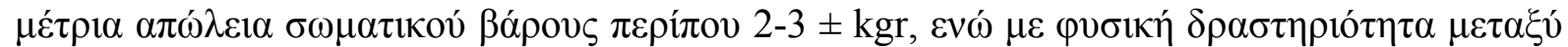

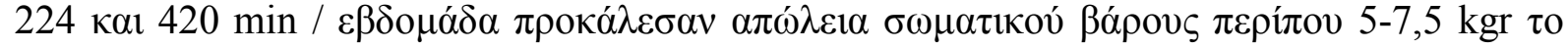

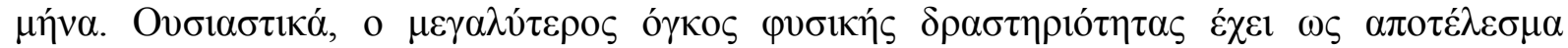

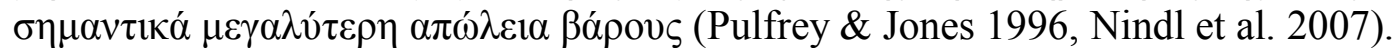

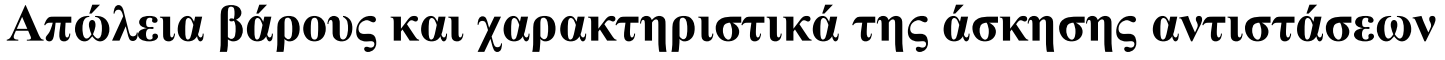

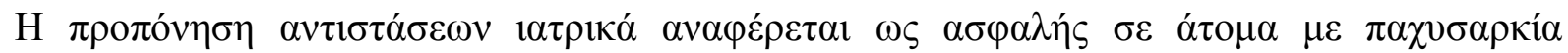

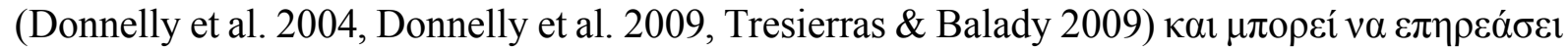

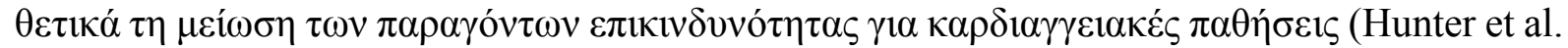

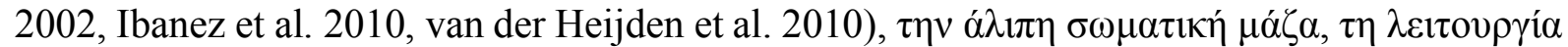

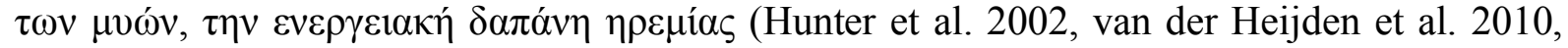
Gillette, Bullough \& Melby 1994, Toth, Beckett \& Poehlman 1999, Hunter, McCarthy \&

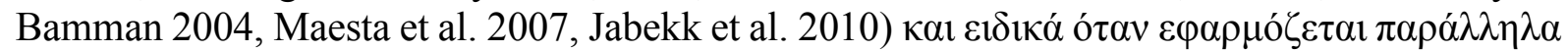

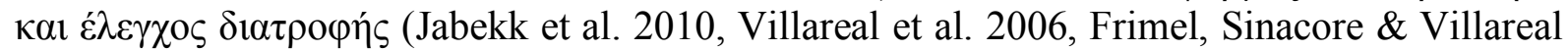

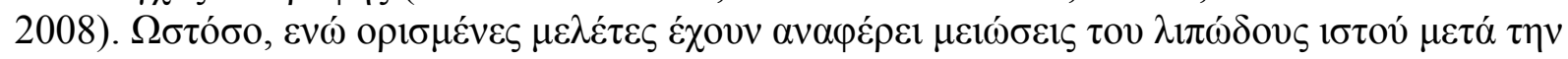
$\pi \rho о \pi \delta ́ v \eta \sigma \eta \alpha v \tau \imath \sigma \tau \alpha ́ \sigma \varepsilon \omega v$ (Hunter et al. 2002, Maesta et al. 2007, Treuth et al. 1994, Treuth et al. 1995, Ross et al. 1996, Sothern et al. 1999, Hagerman et al. 2000, Benson et al. 2008,

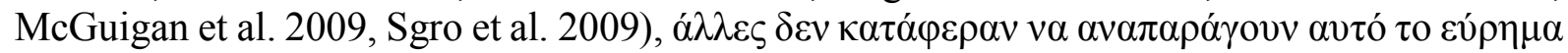
(Schjerve et al. 2008, Donnelly et al. 2009, Donnelly et al. 2004, Jabekk et al. 2010, Hakkinen et al. 2000, Elliott, Sale \& Cable 2002, Teixeira et al. 2003, Shaibi et al. 2006, Bell et al. 2007,

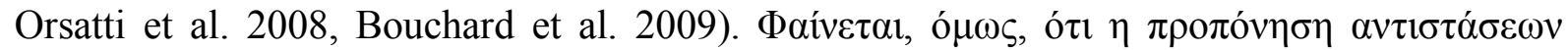

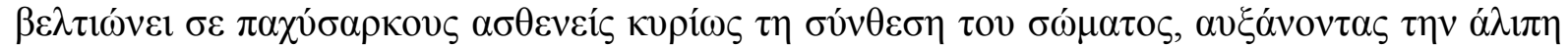

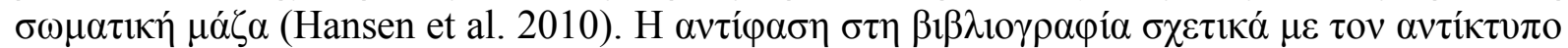

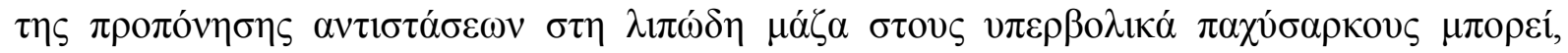

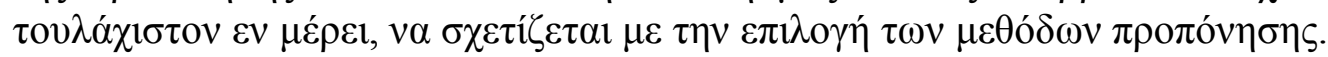

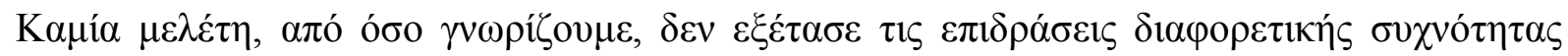

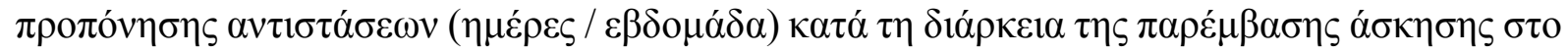
$\lambda ı \pi \omega ́ \delta \eta ~ \iota \sigma \tau o ́ ~ \sigma \varepsilon ~ \pi \alpha \chi v ́ \sigma \alpha \rho \kappa о \nu \varsigma ~ \alpha \sigma \theta \varepsilon v \varepsilon i ́ \varsigma$ (Schjerve et al. 2008, Treuth et al. 1994, Treuth et al.

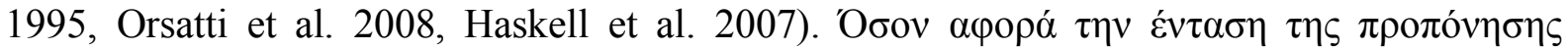

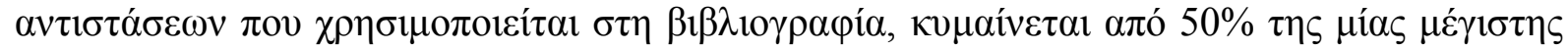




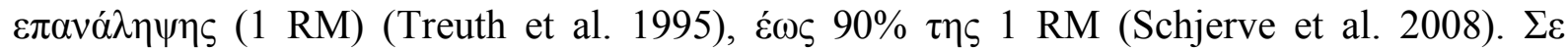

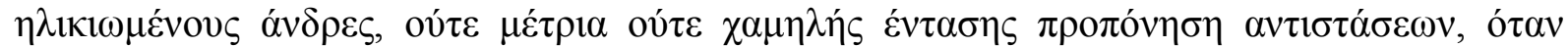

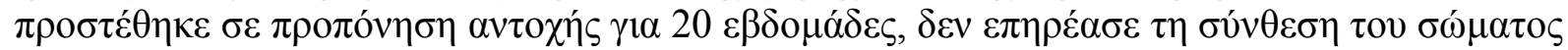

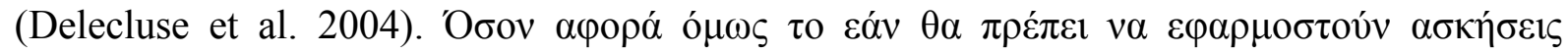

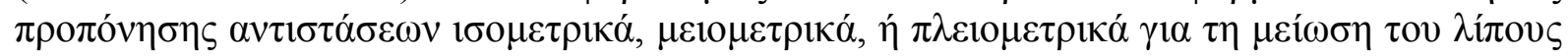

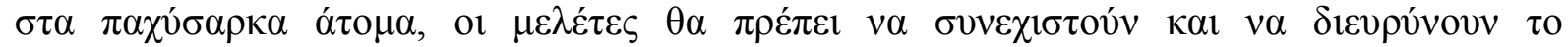

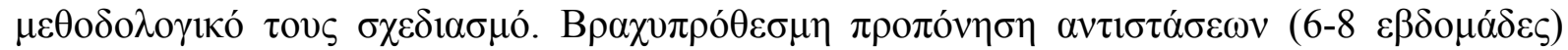

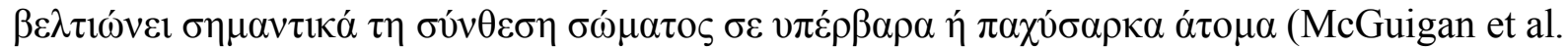

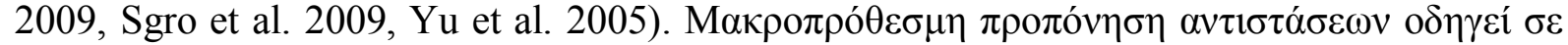

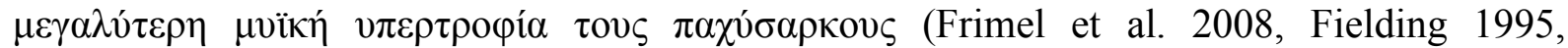

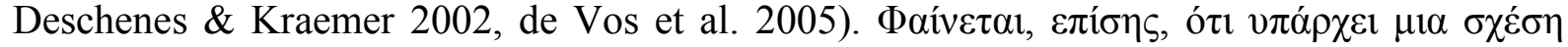

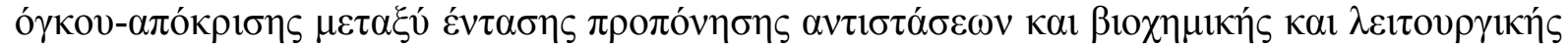

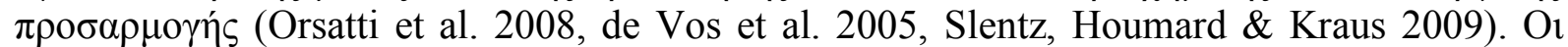

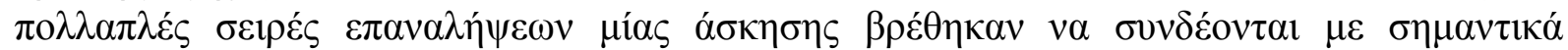

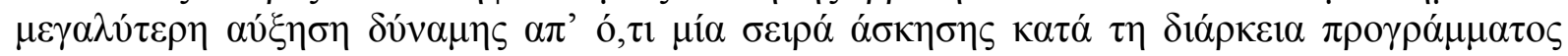

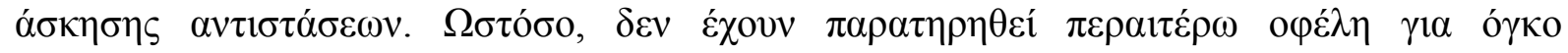

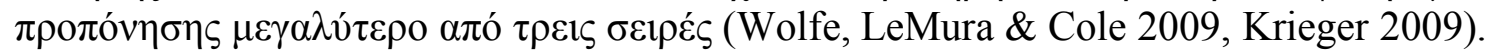

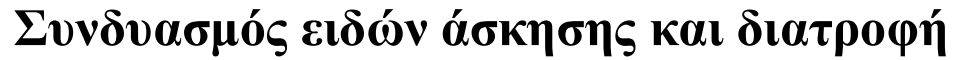

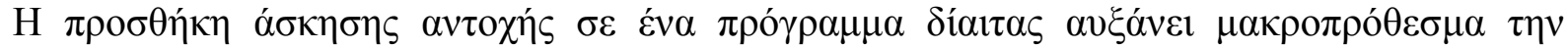

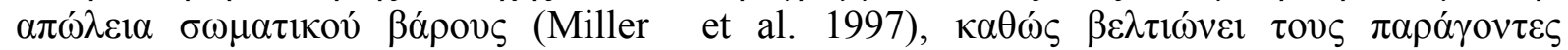

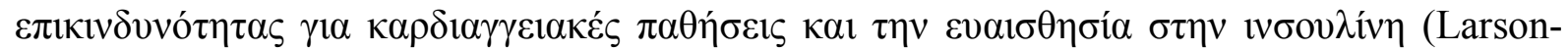

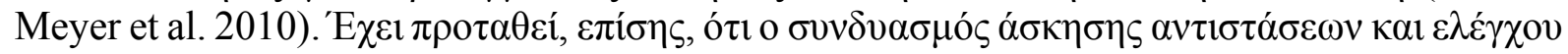

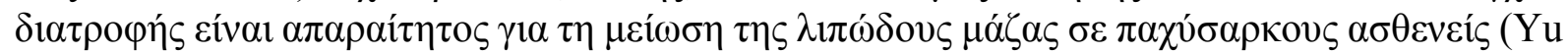

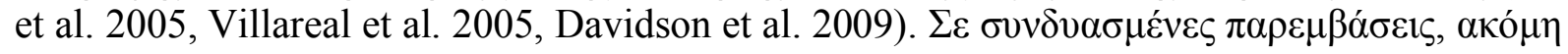

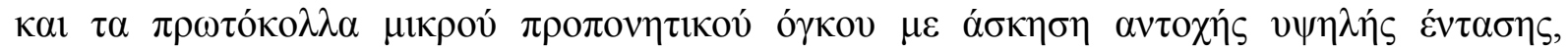

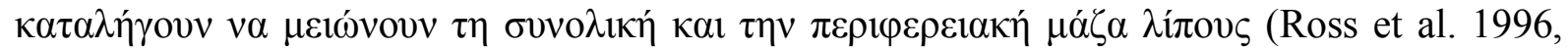

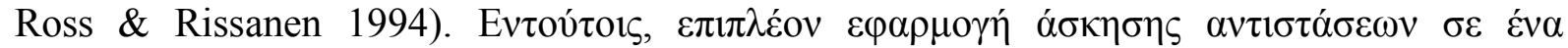

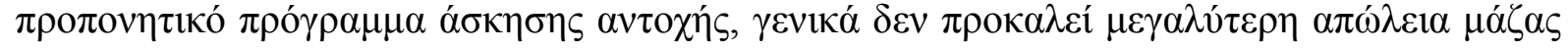
$\lambda i ́ \pi о v \varsigma \sigma \varepsilon \pi \alpha \chi v ́ \sigma \alpha \rho \kappa о v \varsigma \alpha \sigma \theta \varepsilon v \varepsilon i ́ \varsigma$ (Hansen et al. 2010).

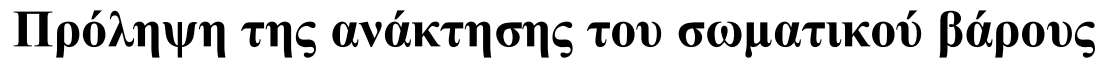

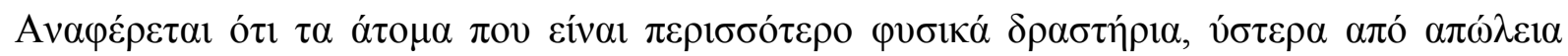

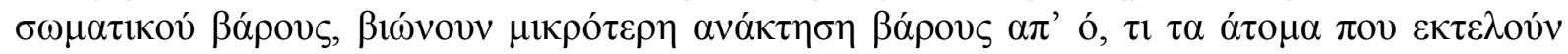

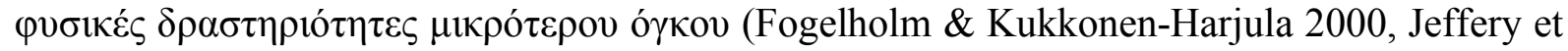

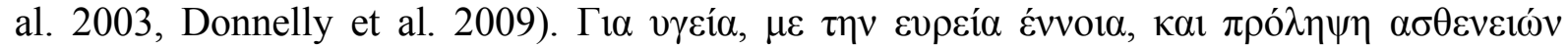

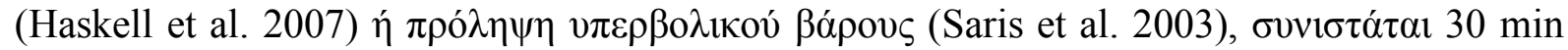

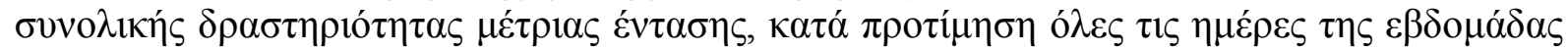

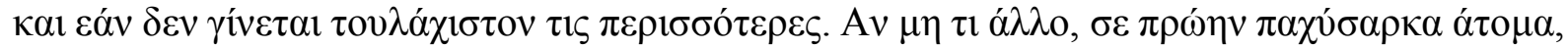

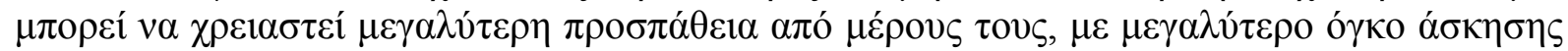

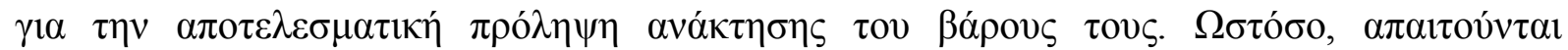

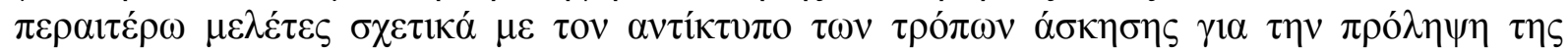

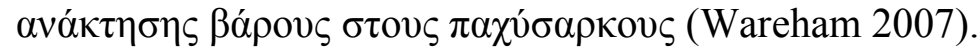

\section{$\Sigma v \mu \pi \varepsilon ́ \rho \alpha \sigma \mu \alpha$}

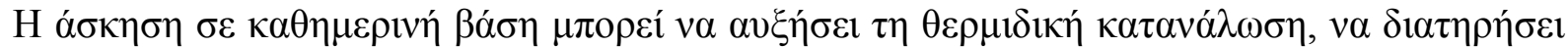

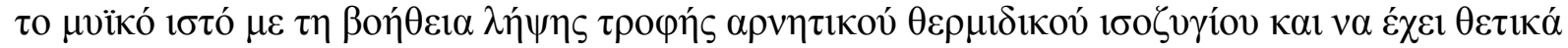

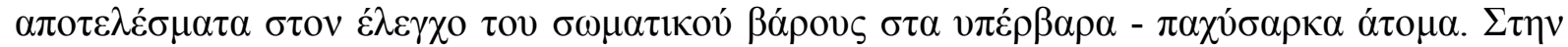

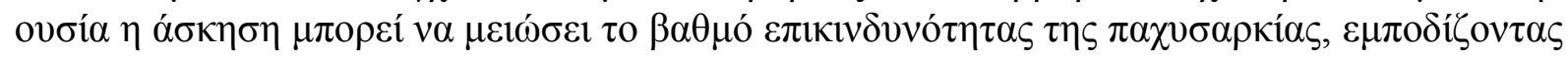




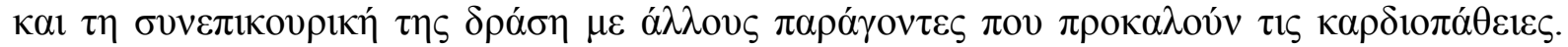

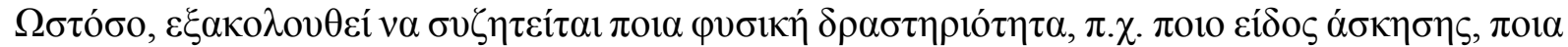

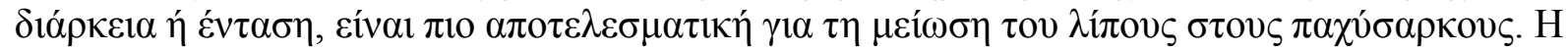

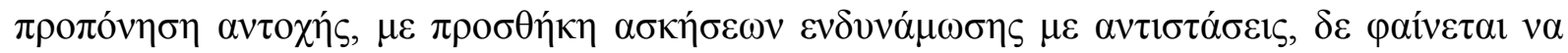

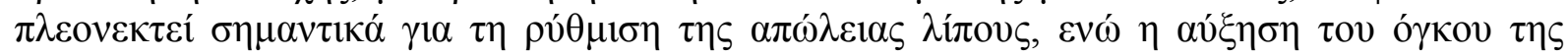

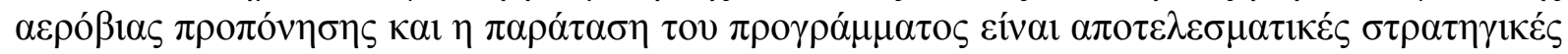

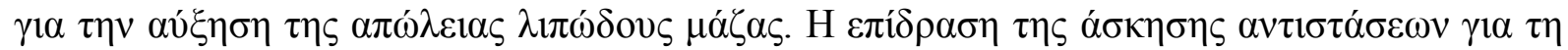

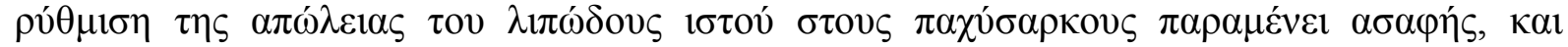

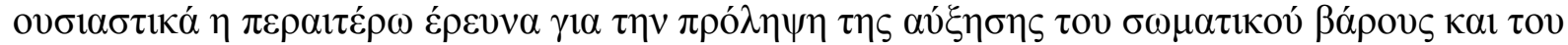

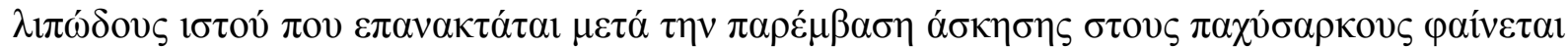

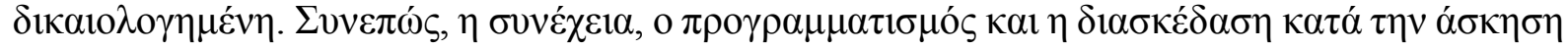

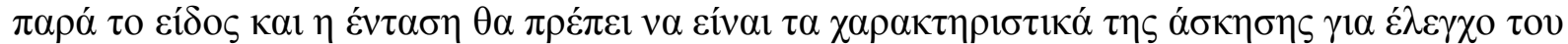

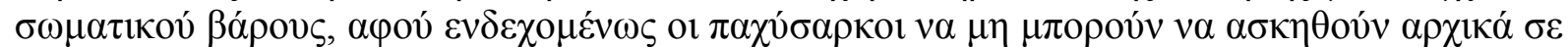

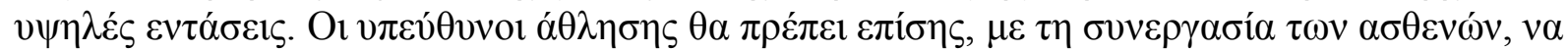

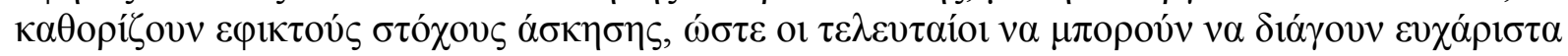

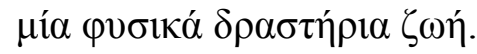

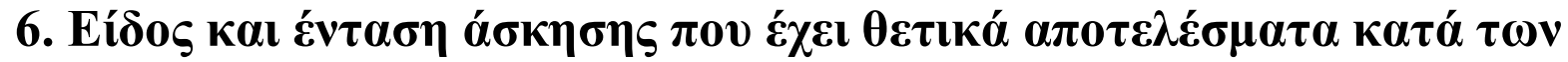

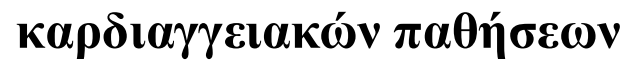

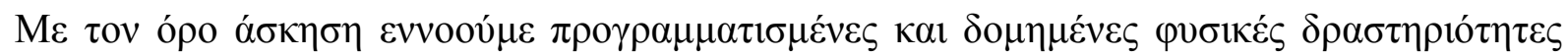

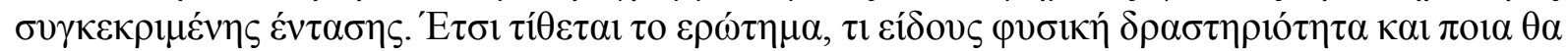

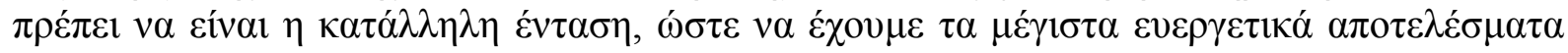

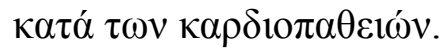

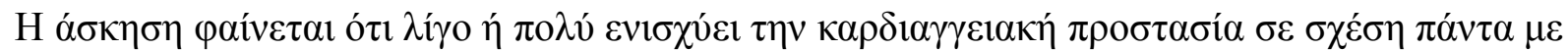

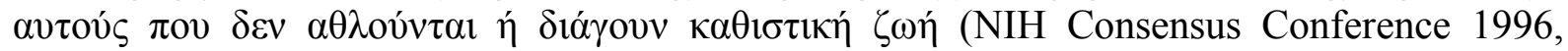

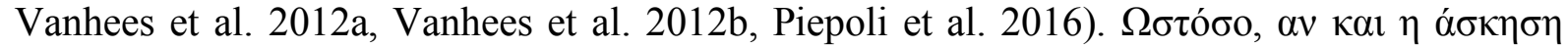

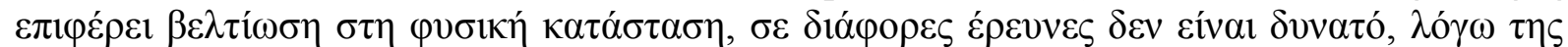

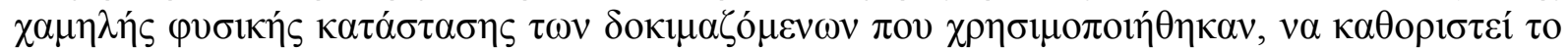

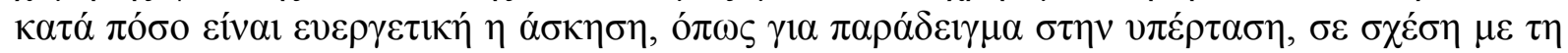

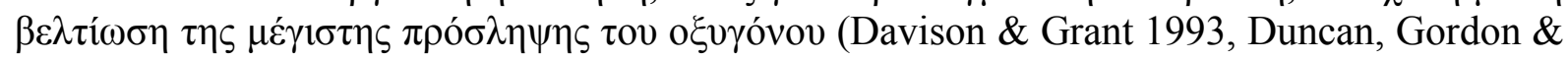

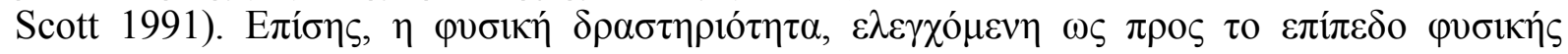

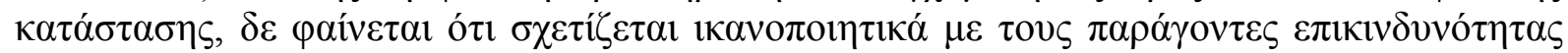

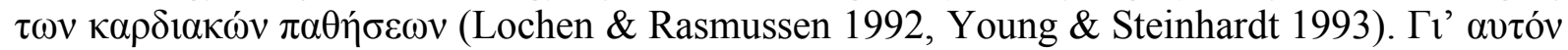

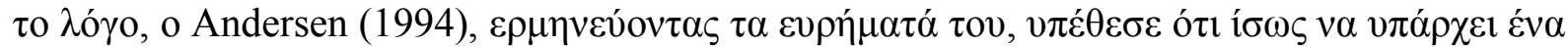

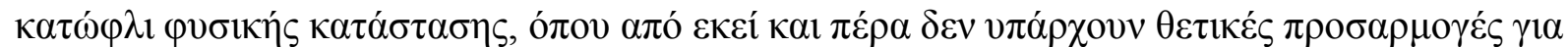

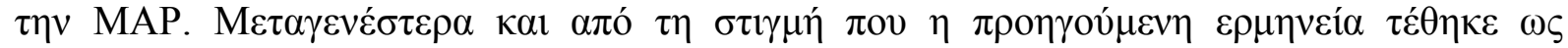

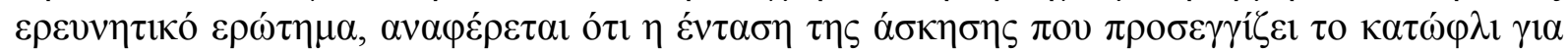

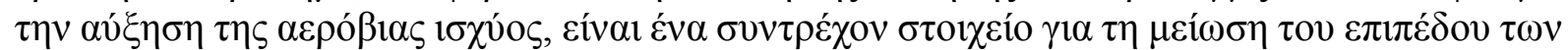

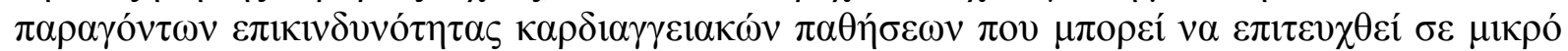

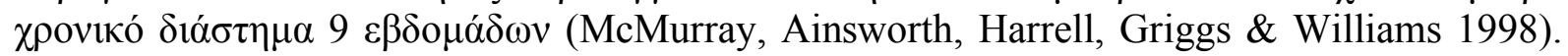

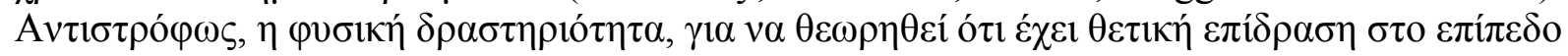

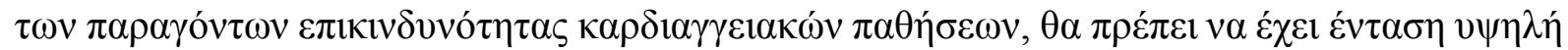

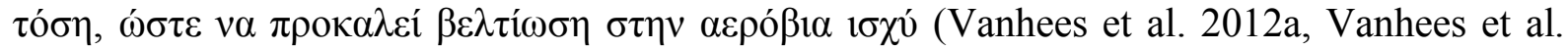

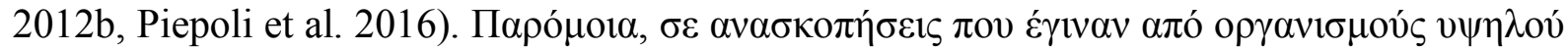

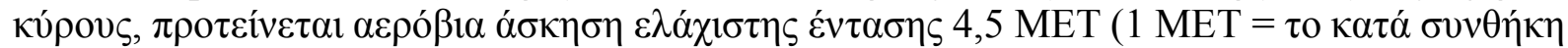

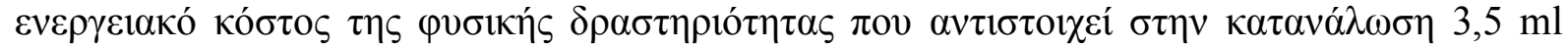

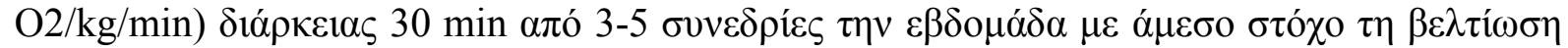

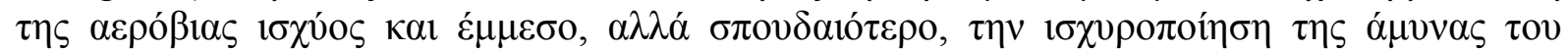

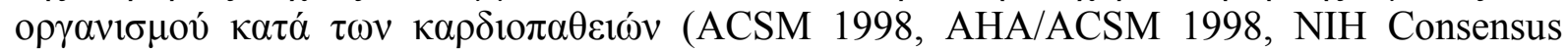




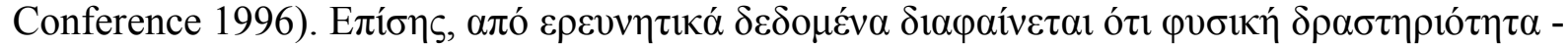

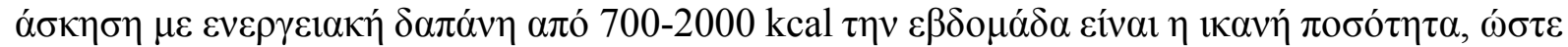

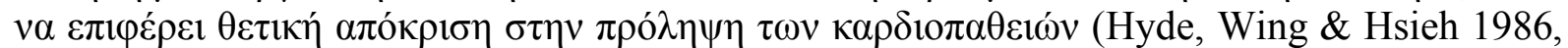
Chung-Cheng \& Paffenbarger, Pate et al. 1995, DHHS 1996, Fletcher et al. 1996, Haennel \& Lemire 2002, Lee 1995, Lee \& Skerrett 2001, Paffenbarger, Vanhees et al. 2012a, Vanhees et al. 2012b, Piepoli et al. 2016).

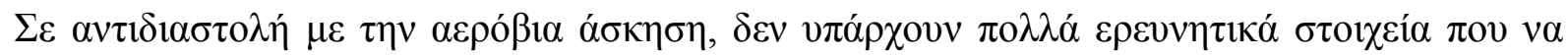

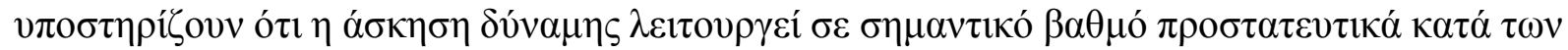

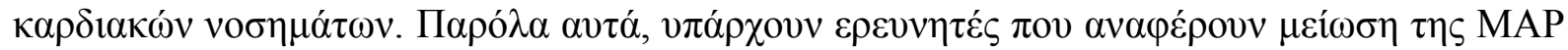

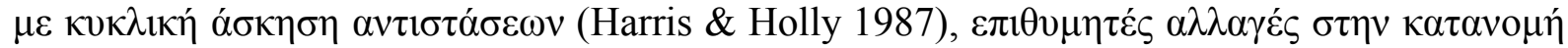

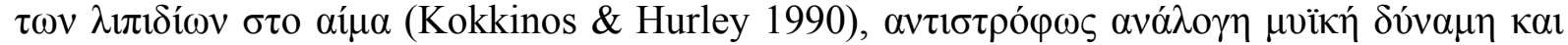

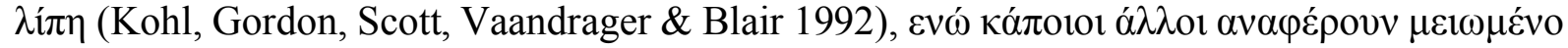

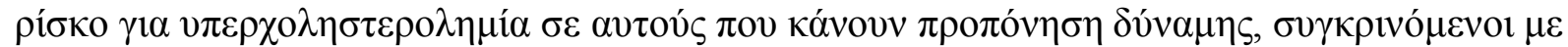

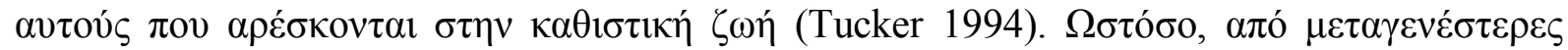

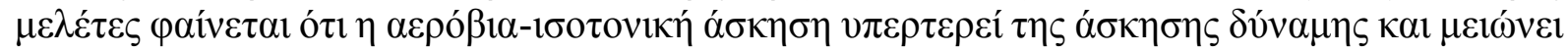

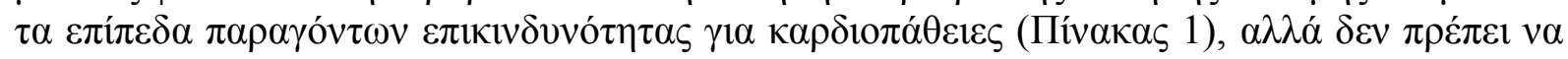

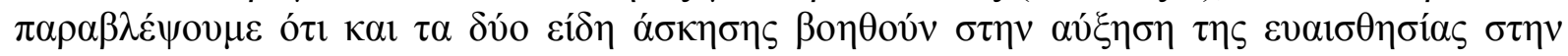

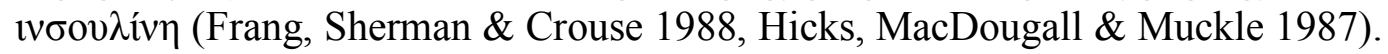

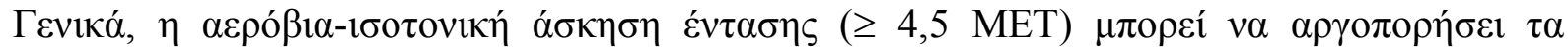

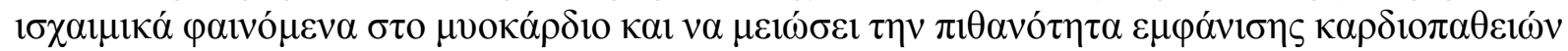

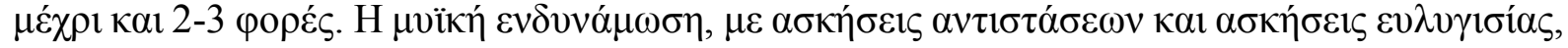

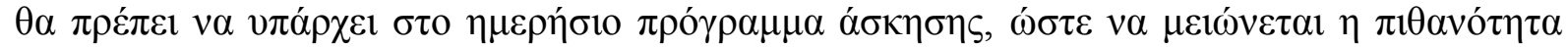

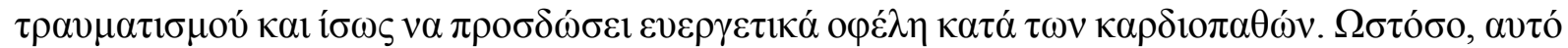

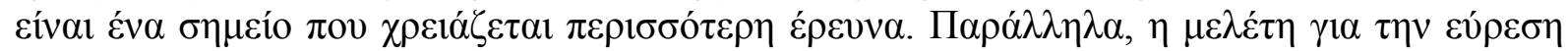

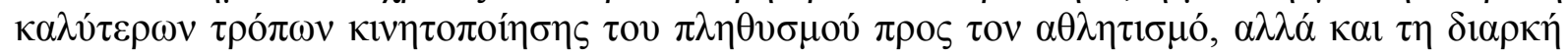

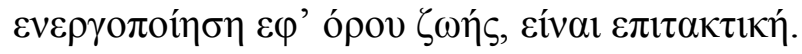

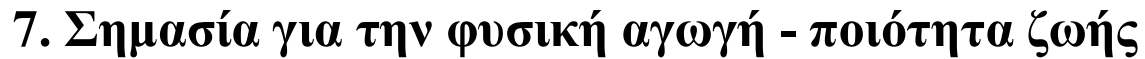

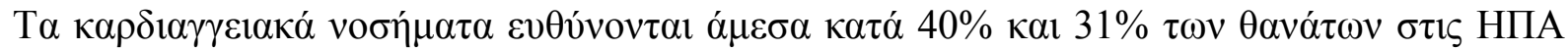

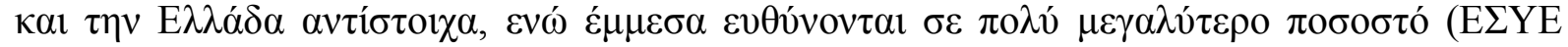

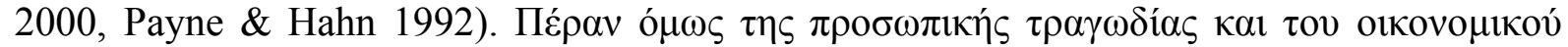

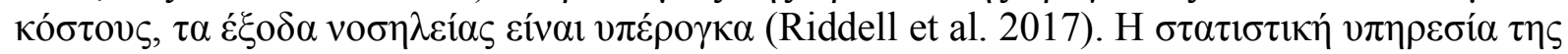

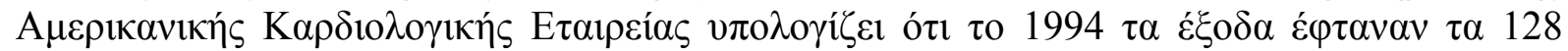

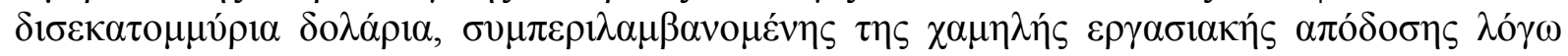

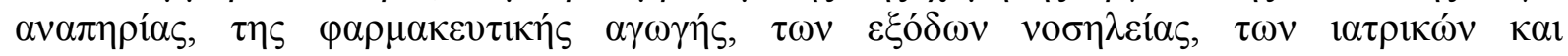

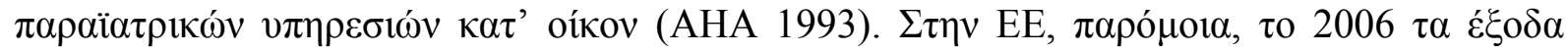

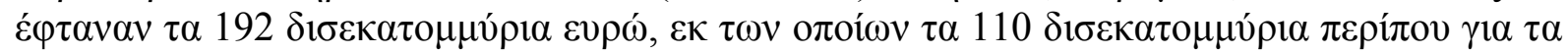

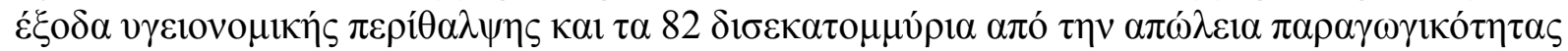

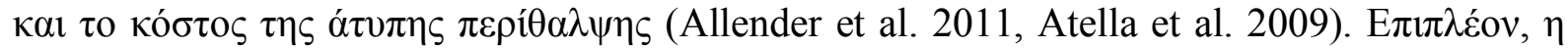

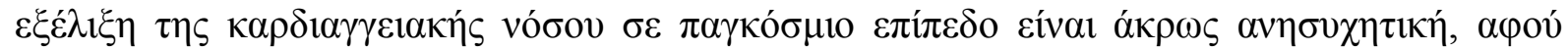

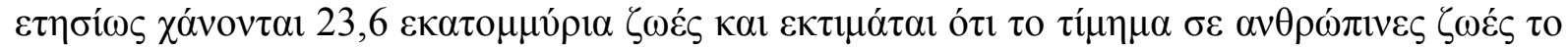

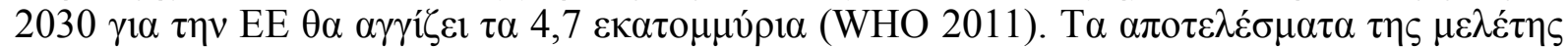

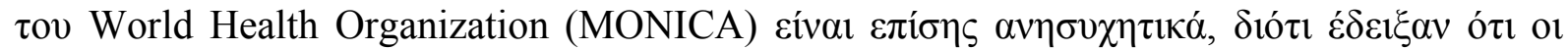

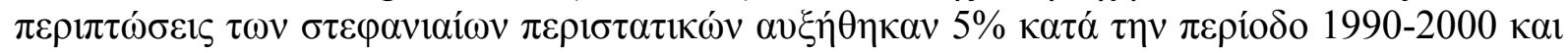

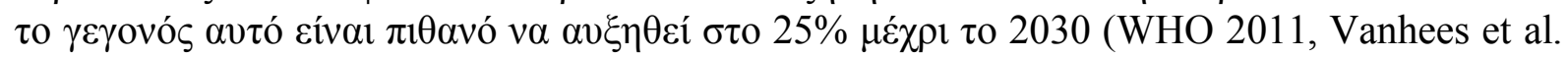

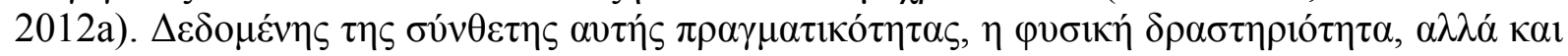

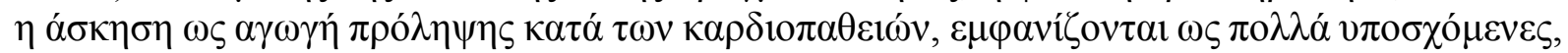

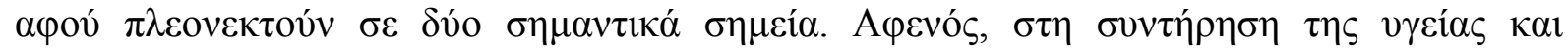




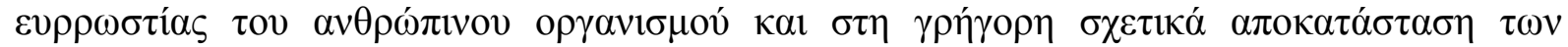

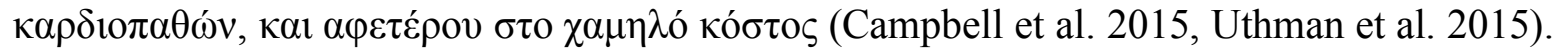

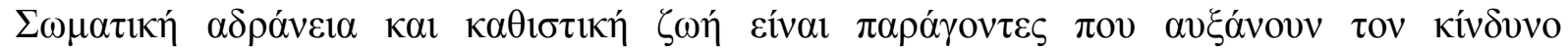

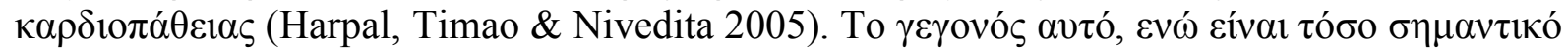

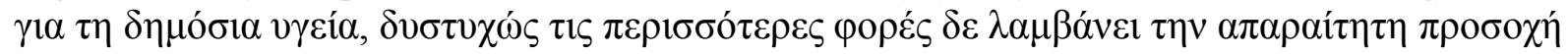

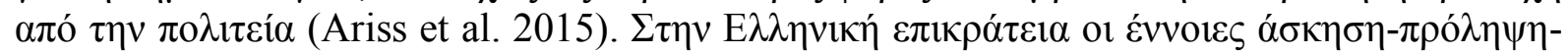

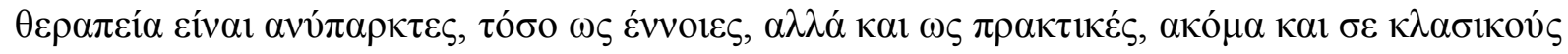

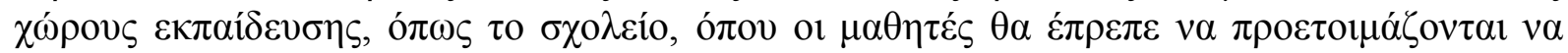

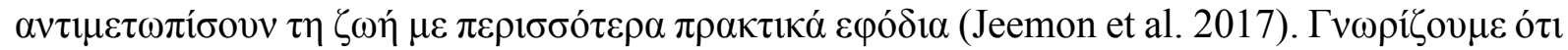

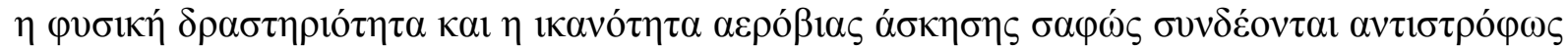

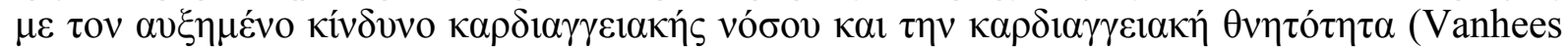

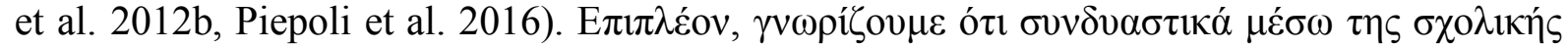

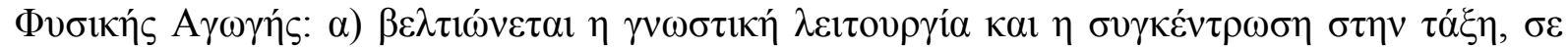

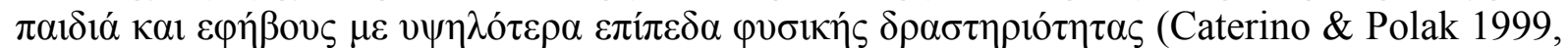

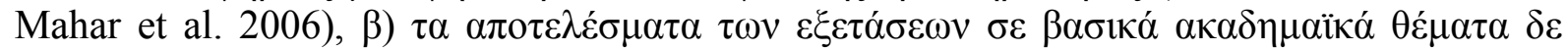

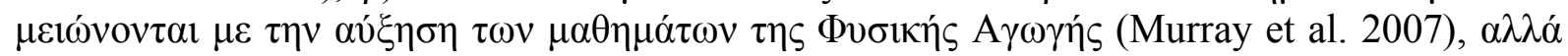

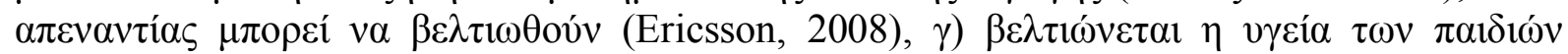

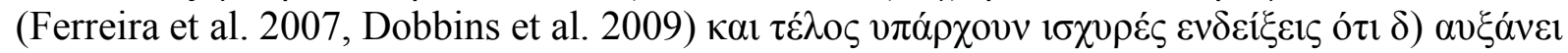

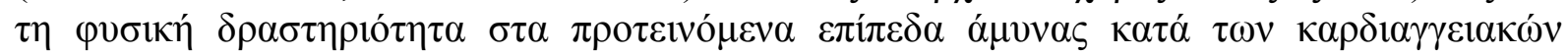

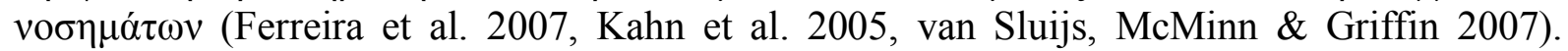

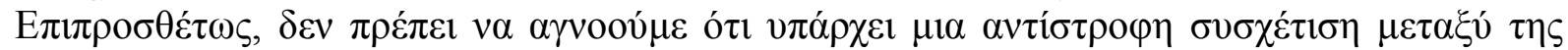

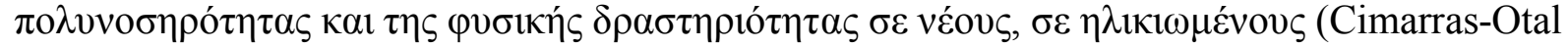

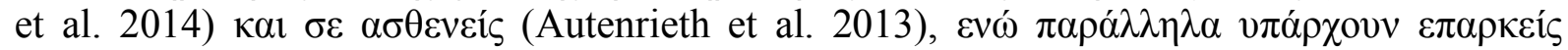

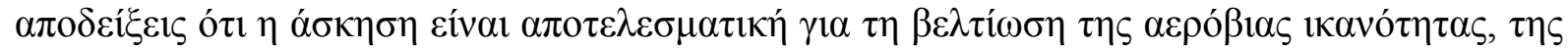

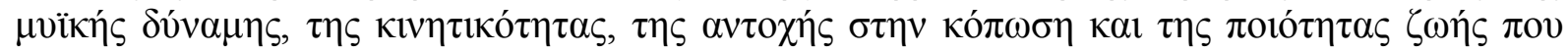

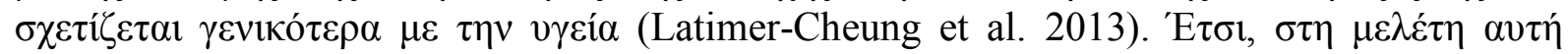

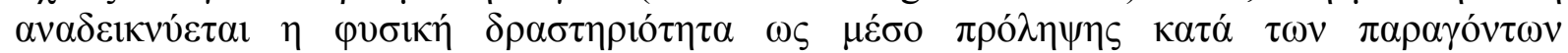

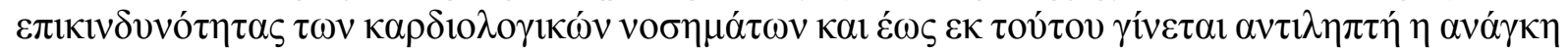

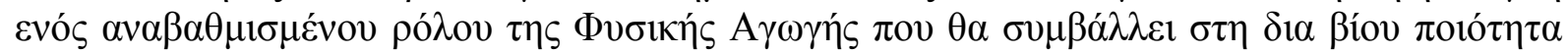

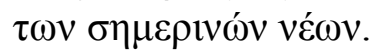

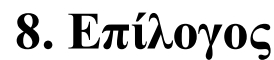

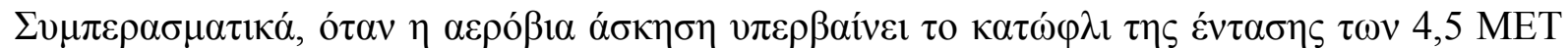

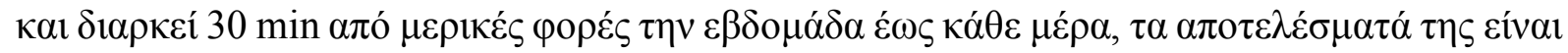

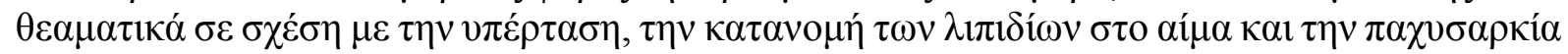

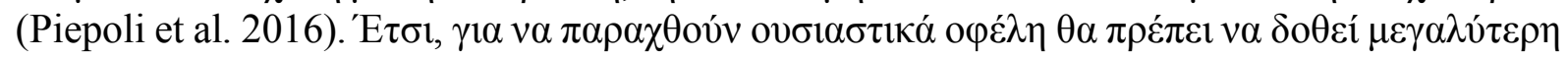

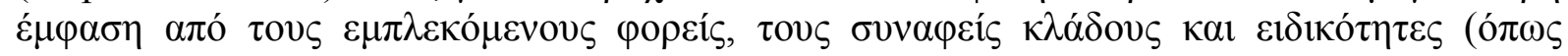

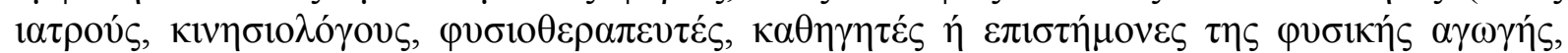

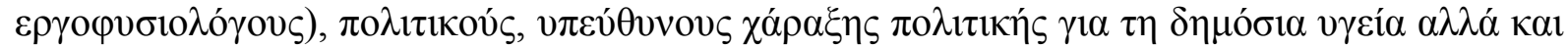

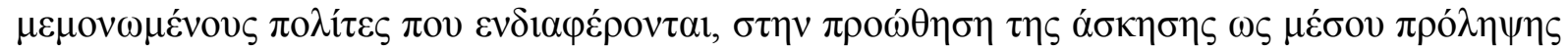

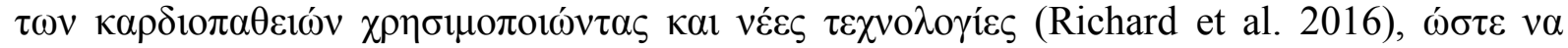

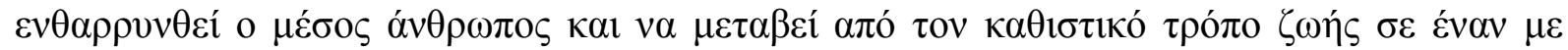

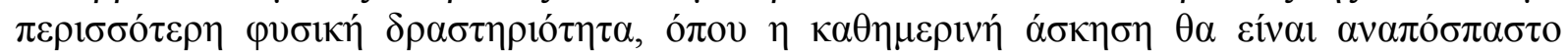

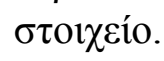

A 


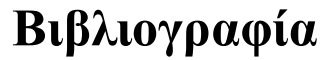

ACSM (2004). Position Stand. Exercise and Hypertension. Medicine \& Science in Sports \& Exercise, 36, 533-553.

ACSM. Position Stand (1998). The recommended quantity and quality of exercise for developing and maintaining cardiorespiratory and muscular fitness, and flexibility in healthy adults. Medicine \& Science in Sports \& Exercise, 30, 975-991.

AHA/ACSM (1998). Joint Position Statement. Recommendations for cardiovascular Screening, stuffing, and emergency policies at health/fitness facilities. Medicine \& Science in Sports \& Exercise, 30, 1009-1018.

Allender, S., Scarborough, P., Peto, V., et al. (2008). European cardiovascular disease statistics.

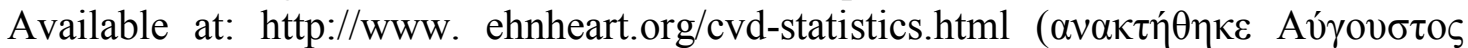
2011).

Allison, P.G. (1981). Failure of exercise to increase high-density lipoprotein cholesterol. Journal of Cardiac Rehabilitation, 1, 257-262.

Altena, T.S., Michaelson, J.L., Ball, S.D., Guilford, B.L. \& Thomas, T.R. (2006). Lipoprotein subfraction changes after continuous or intermittent exercise training. Medicine \& Science in Sports \& Exercise, 38, 367-372.

Alterkruse, E.B. \& Wilmore, J.H. (1973). Changes in blood chemistries following a controlled exercise program. Journal of Occupational Medicine, 15, 110-113.

American Association of Cardiovascular and Pulmonary Rehabilitation (1994). Guidelines for cardiac rehabilitation programs, $2^{\text {nd }}$ edition. Human Kinetics. Books Champaign IL.

American Heart Association (1993). Heart and stroke facts. 1994 statistical supplement. Dallas: The Association.

Ames, R.P. \& Hill, P. (1976). Increase in serum lipids during treatment of hypertension with chlorthalidone. Lanset, I, 721-723.

Andersen L.B. (1994). Blood pressure, physical fitness and physical actcity in 17-year old Danish adolescents. Journal of Internal Medicine, 236, 323-330.

Andersen, L.B. \& Hippe, M. (1996). Coronary heart disease risk factors in the physically active, impact of exercise. Sports Medicine, 22, 213-218.

Araujo, C.G., Duarte, C.V., Goncalves Fde, A., Medeiros, H.B., Lemos, F.A. \& Gouvea, A.L. (2011). Hemodynamic responses to an isometric handgrip training protocol. Arquivos Brasileiros de Cardiologia, 97, 413-419.

Ariss, S.M., Enderby, P.M., Smith, T., Nancarrow, S.A., Bradburn, M.J., Harrop, D., Parker, S.G., McDonnell, A., Dixon, S., Ryan, T., Hayman, A., Campbell, M. (2015). Secondary analysis and literature review of community rehabilitation and intermediate care: an information resource. Health Services and Delivery Research. Southampton (UK): NIHR Journals Library.

Arora, E., Shenoy, S. \& Sandhu, J.S. (2009). Effects of resistance training on metabolic profile of adults with type 2 diabetes. Indian Journal of Medical Research, 129, 515-519.

Arroll, B. \& Beaglehole, R. (1992). Does physical activity lower blood pressure. A critical review of the clinical trials. Journal of Clinical Epidemiology, 45, 439-447.

Asmussen, E. (1981). Similarities and dissimilarities between static and dynamic exercise. Circulation Research, 48 (part 2). I3-I10. 
Atella, V., Brady, A., Catapano, A.L., et al. (2009). Bridging science and health policy in cardiovascular disease: focus on lipid management: A report from a session held during the 7th international symposium on multiple risk factors in cardiovascular diseases: prevention and intervention - health policy. Atherosclerosis Supplements, 10, 3-21.

Autenrieth, C.S., Kirchberger, I., Heier, M., Zimmermann, A.K., Peters A., Döring, A., \& Thorand, B. (2013). Physical activity is inversely associated with multimorbidity in elderly men: results from the KORA-Age Augsburg Study. Preventive Medicine, 57, 17 9.

Ballor, D.L., McCarthy, J.P. \& Wilterdink, E.J. (1990). Exercise intensity does not affect the composition of diet- and exercise-induced body mass loss. The American Journal of Clinical Nutrition, 51, 142-146.

Bell, L.M., Watts, K., Siafarikas, A., Thompson, A., Ratnam, N., Bulsara, M., et al. (2007). Exercise alone reduces insulin resistance in obese children independently of changes in body composition. The Journal of Clinical Endocrinology \& Metabolism, 92, 42304235 .

Bennett, T., Wilcox, R.G. \& Mac Donald, I.A. (1984). Post-exercise reduction of blood pressure in hypertensive men is not due to acute impairment of baroreflex function. Clinical Science, 67, 97-103.

Benson, A.C., Torode, M.E. \& Fiatarone Singh, M.A. (2008). The effect of high-intensity progressive resistance training on adiposity in children: a randomized controlled trial. International Journal of Obesity, 32, 1016-1027.

Benton, M.J. (2005). Safety and efficacy of resistance training in patients with chronic heart failure: research-based evidence. Progress in Cardiovascular Nursing, 20, 17-23.

Berlin, J.A. \& Colditz, G.A. (1990). A meta-analysis of physical activity in the prevention of coronary heart disease. American Journal of Epidemiology, 132, 612-628.

Bertovic, D.A., Waddell, T.K., Gatzka, C.D., Cameron, J.D., Dart, A.M. \& Kingwell, B.A. (1999). Muscular strength training is associated with low arterial compliance and high pulse pressure. Hypertension, 33, 1385-1391.

Bezucha, G.R., Lenser, M.C., Hanson, P.G. \& Nagle, F.J. (1982). Comparison of hemodynamic responces to static and dynamic exercise. Journal of Applied Physiology, 53, 1589-1593.

Bielmann, P. \& Leduc, G. (1979). Effects of metoprolol and propranonolon lipid metabolism. International Journal of Clinical Pharmacology and Biopharmacy, 17, 378-382.

Blair, S.N., Goodyear, N.N., Gibbons, L.W. \& Copper, K.H. (1984). Physical Fitness and incidence of hypertension on healthy normotensive men and women. Journal of the American Medical Association, 252, 487-490.

Bloomgarden, Z.T., Ginsberg-Fellner, F., Rayfield, E.J., Bookman, J. \& Brown, W.V. (1984). Elevated haemoglobin A1c and low-density lipoprotein cholesterol levels in thiazidetreated diabetic patients. American Journal of Medicine, 77, 823-827.

Blumenthal, J.A., Siegel, W.C. \& Applbaum, M. (1991). Failure of exercise to reduce blood pressure in patients with mild hypertension. Results of a randomized controlled trial. Journal of the American Medical Association, 266, 2098-2104.

Blumenthal, J.A., Thyrum, E.T. \& Gullete, E.D. (1995). Do exercise and weight loss reduces blood pressure in patients with mild hypertension? North Carolina Medical Journal, 56, 92-95. 
Bouchard, C. \& Shephard, R.J. (1994). Physical activity, fitness, and health: the model and key concepts". In: Bouchard R, Shephard RJ and Stephens T (eds) Physical activity, fitness, and health: international proceedings and consensus statement. Champaign, IL: Human Kinetics Publishers, pp.77-88.

Bouchard, D.R., Soucy, L., Senechal, M., Dionne, I.J. \& Brochu, M. (2009). Impact of resistance training with or without caloric restriction on physical capacity in obese older women. Menopause, 16, 66-72.

Bray, G.A. (1990). Exercise and Obesity, in. Bouchard C, Shephard RJ, Stephens T, et al. (eds). Exercise, Fitness and Health. Michigan. Human Kinetics Publishers, pp. 497-510.

Breneman, C.B., Polinski, K., Sarzynski, M.A., Lavie, C.J., Kokkinos, P.F., Ahmed, A. \& Sui X. (2016). The Impact of Cardiorespiratory Fitness Levels on the Risk of Developing Atherogenic Dyslipidemia. American Journal of Medicine, 129, 1060-1066

Brown, T., Avenell, A., Edmunds, L.D., Moore, H., Whittaker, V., Avery, L., et al. (2009). Systematic review of long-term lifestyle interventions to prevent weight gain and morbidity in adults. Obesity Reviews, 10, 627-638.

Brownell, K.D., Bachorik, P.S. \& Ayerle, R.S. (1982). Changes in plasma lipid and lipoprotein levels in men and women after a program of moderate exercise. Circulation, 65, 477484.

Brunzell, J.D., Sniderman, A.D., Albers, J.J. \& Kwiterovich, P.O. JR. (1984). Apoproteins B and A-I and coronary artery disease in humans. Arteriosclerosis, 4, 79-83.

Buck, C., Donner, A.P. (1985). Isometric occupational exercise and the incidence of hypertension. Journal of Occupational Medicine, 27, 370-372.

Cade, R., Mars, D.,Wagemaker, H., Zauner, C., Packer, D., Privette, M., Cade, M., Peterson, J. $\&$ Hood-Lewis, D. (1984). Effect of aerobic exercise training on patients with systemic arterial hypertension. American Journal of Medicine, 77, 785-790.

Campbell, F., Holmes, M., Everson-Hock, E., Davis, S., Buckley Woods, H., Anokye, N., Tappenden, P., Kaltenthaler, E., (2015). A systematic review and economic evaluation of exercise referral schemes in primary care: a short report. Health Technology Assessment. 19(60):1-110. doi: 10.3310/hta19600.

Carlson, D.J., Dieberg, G., Hess, N.C., Millar, P.J. \& Smart, N.A. (2014). Isometric exercise training for blood pressure management: a systematic review and meta-analysis. Mayo Clinic Proceedingseedings, 89, 327-334.

Carnethon, M.R., Gidding, S.S., Nehgme, R., et al. (2003). Cardiorespiratory fitness in young adulthood and the development of cardiovascular disease risk factors. Journal of the American Medical Association, 290, 3092-100.

Carroll, S. \& Dudfield, M. (2004). What is the relationship between exercise and metabolic abnormalities? A review of the metabolic syndrome. Sports Medicine, 34(6), 371-418.

Caspersen, C.J., Powell, K.E. \& Christensen, G.M. (1985). Physical activity, exercise, and physical fitness: definitions and distinctions for health-related research. Public Health Reports, 100, 126-131.

Caterino, M.C. \& Polak, E.D. (1999). Effects of two types of activity on the performance of second-, third-, and fourth-grade students on a test of concentration. Perceptual and Motor Skills, 89, 245-248. 
Chapman, M.J., Ginsberg, H.N., Amarenco, P., Andreotti, F., Boren, J., Catapano, A.L., et al. (2011). For the European Atherosclerosis Society Consensus Panel. Triglyceriderich lipoproteins and high-density lipoprotein cholesterol in patients at high risk of cardiovascular disease: evidence and guidance for management. European Heart Journal, 32, 1345-1361.

Church, T.S., Finley, C.E., Earnest, C.P., Kampert, J.B., Gibbons, L.W. \& Blair, S.N. (2002). Relative associations of fitness and fatness to fibrinogen, white blood cell count, uric acid and metabolic syndrome. International Journal of Obesity and Related Metabolic Disorders, 26, 805-813.

Church, T.S., Thomas, D.M., Tudor-Locke, C., Katzmarzyk, P.T., Earnest, C.P., Rodarte, R.Q., Martin, C.K., Blair, S.N. \& Bouchard, C. (2011). Trends over 5 decades in U.S. occupation-related physical activity and their associations with obesity. PLoS One, 6,25. https://doi.org/10.1371/journal.pone.0019657

Cimarras-Otal C., Calderón-Larrañaga A., Poblador-Plou B., González-Rubio F., Luis A Gimeno-Feliu, Arjol-Serrano J.L., \& Prados-Torres A. (2014). Association between physical activity, multimorbidity, self-rated health and functional limitation in the

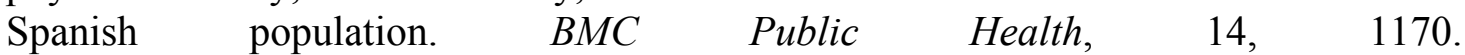
http://www.biomedcentral.com/1471-2458/14/1170

Cooper, S.P., Hardy, R.J., Labarthe, D.R., Hawkins, C.M., Smith, E.O. \&, Blaufox, M.D. (1988). The relation between degree of blood pressure reduction and mortality among hypertensives in the Hypertension Detection and Follow-up Program. American Journal of Epidemiology, 127, 387-403.

Cornelissen, V.A. \& Fagard, R.H. (2005b) Effect of resistance training on resting blood pressure: a meta-analysis of randomized controlled trials. Journal of Hypertension, 23:251-259.

Cornelissen, V.A., \& Smart, N.A. (2013). Exercise training for blood pressure: a systematic review and meta-analysis. Journal of the American Heart Association, 2, e004473.

Cornelissen, V.A., Buys, R. \& Smart, N.A. (2013). Endurance exercise beneficially affects ambulatory blood pressure: a systematic review and meta-analysis. Journal of Hypertension, 31, 639-648.

Cornelissen, V.A., Fagard, R.H. (2005). Effects of endurance training on blood pressure, blood pressure-regulating mechanisms, and cardiovascular risk factors. Hypertension, 46, 667675.

Cornelissen, V.A., Fagard, R.H., Coeckelberghs, E. \& Vanhees, L. (2011). Impact of resistance training on blood pressure and other cardiovascular risk factors: a meta-analysis of randomized, controlled trials. Hypertension, 58, 950-958.

Danner, S.A., Wieling, W., Havekes, L., Leuven, J.G., Smit, E.M. \& Dunning, A.J. (1984). "Effect of physical exercise on blood lipids and adipose tissue composition in young healthy men. Atherosclerosis, 53, 83-90.

Davidson, L.E., Hudson, R., Kilpatrick, K., Kuk, J.L., McMillan, K., Janiszewski, P.M., et al. (2009). Effects of exercise modality on insulin resistance and functional limitation in older adults: a randomized controlled trial. Archives of Internal Medicine, 169, 122-131.

Davison, R.C.R. \& Grant, S. (1993). Is walking sufficient exercise for health? Sports Medicine, 16, 369-373. 
de Vos, N.J., Singh, N.A., Ross, D.A., Stavrinos, T.M., Orr, R. \& Fiatarone, Singh, M.A. (2005). Optimal load for increasing muscle power during explosive resistance training in older adults. The Journal of Gerontology, Series A: Biological Sciences and Medical Sciences, 60, 638-647.

Delecluse, C., Colman, V., Roelants, M., Verschueren, S., Derave, W., Ceux, T., et al. (2004). Exercise programs for older men: mode and intensity to induce the highest possible health-related benefits. Preventive Medicine, 39, 823-833.

Department Of Health And Human Services (1996). Physical Activity and Health. A Report of the Surgeon General. Atlanta. U.S. Department of Health and Human Services, Centers for Disease Control and Prevention, National Center for Chronic Disease Prevention and Health Promotion.

Deschenes, M.R. \& Kraemer, W.J. (2002). Performance and physiologic adaptations to resistance training. American Journal of Physical Medicine \& Rehabilitation, 81(Suppl 11), S3-S16.

Dobbins, M., De Corby, K,. Robeson, P., et al. (2009). School-based physical activity programs for promoting physical activity and fitness in children and adolescents aged 6-18. The Cochrane Database of Systematic Reviews, (1): CD007651.

Donnelly, J.E., Blair, S.N., Jakicic, J.M., Manore, M.M., Rankin, J.W. \& Smith, B.K. (2009). American College of Sports Medicine Position Stand. Appropriate physical activity intervention strategies for weight loss and prevention of weight regain for adults. Medicine \& Science in Sports \& Exercise, 41, 459-471.

Donnelly, J.E., Smith, B., Jacobsen, D.J., Kirk, E., Dubose, K., Hyder, M., Bailey, B. \& Washburn, R. (2004). The role of exercise for weight loss and maintenance. Best Practice \& Research: Clinical Gastroenterology, 18(6), 1009-29.

Dubbert, P.M., Carithers, T., Sumner, A.E., Barbour, K.A., Clark, B.L., Hall, J.E. \& Crook, E.D. (2002). Obesity, physical inactivity, and risk for cardiovascular disease. American Journal of Medicine Sci, 324(3), 116-26.

Duncan, G.E., Anton, S.D., Sydeman, S.J., Newton, Jr R.L., Corsica, J.A., Durning, P.E., et al. (2005). Prescribing exercise at varied levels of intensity and frequency: a randomized trial. Archives of Internal Medicine, 165, 2362-2369.

Duncan, J.J., Gordon, N.F. \& Scott, C.B. (1991). Women walking for health and fitness". Journal of the American Medical Association, 266, 3295-3299.

Durstine, J.L., Grandjean, P.W., Davis, P.G., Ferguson, M.A., Alderson, N.L. \& DuBose, K.D. (2001). Blood lipid and lipoprotein adaptations to exercise". Sports Medicine, 31, 10331062 .

Eckel, R.H., Jakicic, J.M., Ard, J.D., et al. (2014). 2013 AHA/ACC guideline on lifestyle management to reduce cardiovascular risk: a report of the American College of Cardiology/American Heart Association Task Force on Practice Guidelines. Journal of the American College of Cardiology, 63, 2960-84.

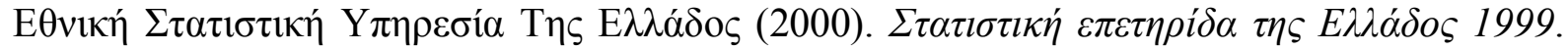
A $\theta \dot{n} v \alpha$.

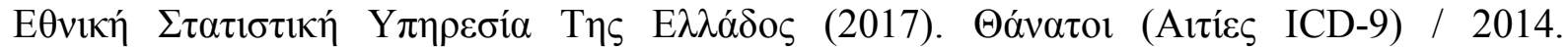

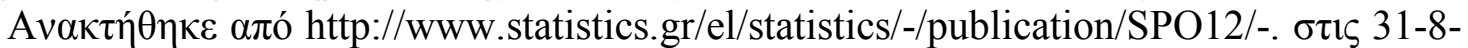
2017. 
Elliott, K.J., Sale, C. \& Cable, N.T. (2002). Effects of resistance training and detraining on muscle strength and blood lipid profiles in postmenopausal women. British Journal of Sports Medicine, 36, 340-344.

Ericsson, I. (2008). Motor skills, attention and academic achievements - an intervention study in school year 1-3. Br Educ Res $J, 34,301-313$.

Expert Panel On Detection, Evaluation And Treatment Of High Blood Cholesterol In Adults [Adult Treatment Panel II] (1993). Second report of the National Cholesterol Education Program [NCEP] Men Screened for MR-FIT Program. NIH Publication, No.39. 361, 662.

Expert Panel on Detection, Evaluation, and Treatment of High Blood Cholesterol in Adults (2001). Executive Summary of the Third Report of the National Cholesterol Education Program (NCEP) Expert Panel on Detection, Evaluation, and Treatment of High Blood Cholesterol in Adults (Adult Treatment Panel III). Journal of the American Medical Association, 285, 2486-2497.

Fagard, R.H. \& Cornelissen, V.A. (2007). Effect of exercise on blood pressure control in hypertensive patients. European Journal of Cardiovascular Prevention and Rehabilitation, 14, 12-17.

Farnett, L., Mulrow, C.D., Linn, W.D., Lucey, C.R. \& Tuley, M.R. (1991). The J-curve phenomenon and the treatment of hypertension. Is there a point beyond which pressure reduction is dangerous? Journal of the American Medical Association, 265, 489-495.

Farrell, P.A., Maksud, M.G., Pollock, M.L., Foster, C., Anholm, J., Hare, J. \& Leon, A.S. (1982). A comparison of plasma cholesterol, triglycerides, and high-density lipoprotein cholesterol in speed skaters, weight-lifters, and non athletes. European Journal of Applied Physiology, 48, 77-82.

Ferreira, I., van der Horst, K., Wendel-Vos, W., et al. (2007). Environmental correlates of physical activity in youth - a review and update. Obesity Reviews, 8, 129-154.

Fielding, R.A. (1995). The role of progressive resistance training and nutrition in the preservation of lean body mass in the elderly. Journal of the American College of Nutrition, 14, 587-594.

Fleg, J.L., Morrell, C.H., Bos, A.G., et al. (2005). Accelerated longitudinal decline of aerobic capacity in healthy older adults. Circulation, 112, 674-682.

Fletcher, B., Berra, K., Ades, P., et al. (2005). Managing abnormal blood lipids: a collaborative approach". Circulation, 112, 184-3209.

Fletcher, G.F., Balady, G., Blair, S.N., Blumenthal, J., Caspersen, C., Chaitman, B., Epstein S., Sivarajan Froelicher E.S., Froelicher V.F., Pina I.L. \& Pollock M.L. (1996). Statement on exercise. Benefits and recommendations for physical activity programs for all Americans. A statement for health professionals by the Committee on Exercise and Cardiac Rehabilitation of the Council on Clinical Cardiology, American Heart Association. Circulation, 94, 857-862.

Fogelholm, M. \& Kukkonen-Harjula, K. (2000). Does physical activity prevent weight gain-a systematic review. Obesity Reviews, 1, 95-111.

Frang, C.L., Sherman, W.M., Crouse, S.F. (1988). Exercise modality and selected coronary risk factors. a multivariate approach. Medicine \& Science in Sports \& Exercise, 20, 455-462. 
Franklin, B.A., Durstine, J.L., Roberts, C.K. \& Barnard, R.J. (2014). Impact of diet and exercise on lipid management in the modern era. Best Practice \& Research: Clinical Endocrinology \& Metabolism, 28, 405-421.

Fraser, G.E., Philips, R.L. \& Harris, R. (1983). Physical fitness and blood pressure in school children. Circulation, 67, 405-412.

Freyman, J.F. (1982). Effects of 12 weeks of exercise training on plasma lipids and apoproteins in middle aged men (abstract). Medicine \& Science in Sports \& Exercise, 14, 103.

Frimel, T.N., Sinacore, D.R. \& Villareal, D.T. (2008). Exercise attenuates the weight-lossinduced reduction in muscle mass in frail obese older adults. Medicine \& Science in Sports \& Exercise, 40, 1213-1219.

Giada, F., Baldo-Enzi, G., Baiocchi, M.R., Zuliani, G., Vitale, E. \& Fellin, R. (1991). Specialized physical training programs: effects on serum lipoproteins cholesterol, apoproteins AI and B and lipolytic enzyme activities. The Journal of Sports Medicine and Physical Fitness, 31, 196-203.

Giada, F., Vigna, G.B., Vitale, E., Baldo-Enzi, G. \& Fellin, R. (1995). Effect of age on the response of blood lipids, body composition, and aerobic power in physical conditioning and deconditioning. Metabolism, 44, 161-165.

Gillette, C.A., Bullough, R.C. \& Melby, C.L. (1994). Postexercise energy expenditure in response to acute aerobic or resistive exercise. International Journal of Sport Nutrition, 4, 347-360.

Goldberg, L. \& Elliot, D.L. (1987). The effect of exercise on lipid metabolism in men and women. Sports Medicine, 4, 307-321.

Goldeberg, L. \& Elliot, D. (1994). Exercise as treatment for essential hypertension. In; Goldeberg L, Elliot D. F.A. Davis Company (eds) Exercise for prevention and treatment of illness, Philadelfia, pp. 27-47.

Goldeberg, L., Elliot, D.L., Schutz R.W. \& Kloster F.E. (1984). Changes in lipid and lipoprotein levels after weight training. Journal of the American Medical Association, 252, 504-526.

Gordon, D.J. \& Rifkind, B.M. (1989). The high intensity lipoprotein. The clinical implications of recent studies. The New England Journal of Medicine, 321, 1311-1316.

Gordon, D.J., Witztum, J.L., Hunninghake, D., Gates, S. \& Glueck, C.J. (1983). Habitual physical activity and high density lipoprotein cholesterol in men with primary hypercholesterolemia. The Lipid Research Clinics Coronary Primary Prevention Trial. Circulation, 67, 512-520.

Grassi, G., Seravalle, G., Calhoun, D.A. \& Mancia, G. (1994). Physical training and baroreceptor control of sympathetic nerve activity in humans. Hypertension, 23, 294301.

Grilo, C.M. \& Brownell, K.D. (2001). Interventions for Weight Management, in. Lippincot Williams \& Wilkins (eds), ACSM's Resource Manual for Guidelines Exercise Testing and Prescription, $4^{\text {th }}$ edition. Philadelfia, pp 584-591.

Gyntelberg, F., Brennan, R., Holloszy, J.O., Schonefeld, G., Rennie, M.J. \& Weidman, S.W. (1977). Plasma triglyceride lowering by exercise despite increased food intake in patients with type IV hyperlipoproteinemia. The American Journal of Clinical Nutrition, 30, 716-720. 
Hackam, D.G., Khan, N.A., Hemmelgarn, B.R. et al. 2010. The 2010 Canadian Hypertension Education Program recommendations for the management of hypertension: Part 2 therapy. Canadian Journal of Cardiology, 26, 249-258.

Haennel, R.G. \& Lemire, F. (2002). Physical activity to prevent cardiovascular disease. How much is enough? Canadian Family Physician, 48, 65-71.

Hagberg, J.M. (1990). Exercise fitness and hypertension, In. Bouchard et al. (eds). Exercise Fitness and Health. A Consensus Current Knowledge. Human Kinetics Books, Champaign IL.

Hagberg, J.M. (1995). Physical activity, physical fitness and blood pressure. NIH Consensus Development Conference. Physical Activity and Cardiovascular Health. Bethesda, MD. National Institutes of Health, pp. 69-71.

Hagberg, J.M., \& Seals, D.R. (1986). Exercise training and hypertension. Acta Medica Scandinavica, Supplementum, 711(suppl), 131-136.

Hagberg, J.M., Ehsani, A.A., Goldring, D., Hernandez, A., Sinacore, D.R. \& Holloszy, J.O. (1984). Effect of weight training on blood pressure and hemodynamics in hypertensive adolescents. Journal of Pediatrics, 104, 147-151.

Hagberg, J.M., Park, J.J. \& Brown, M.D. (2000). The role of exercise training in the treatment of hypertension: an update. Sports Medicine, 30, 193-206.

Hagerman, F.C., Walsh, S.J., Staron, R.S., Hikida, R.S., Gilders, R.M., Murray, T.F., et al. (2000). Effects of high-intensity resistance training on untrained older men. I. Strength, cardiovascular, and metabolic responses. The Journal of Gerontology, Series A: Biological Sciences and Medical Sciences, 55, B336-B346.

Hakkinen, K., Pakarinen, A., Kraemer, W.J., Newton, R.U. \& Alen, M. (2000). Basal concentrations and acute responses of serum hormones and strength development during heavy resistance training in middle-aged and elderly men and women. The Journal of Gerontology, Series A: Biological Sciences and Medical Sciences, 55, B95-B105.

Halbert, J.A., Silagy, C.A., Finucane, P., Withers, R.T. \& Hamdorf, P.A. (1999). Exercise training and blood lipids in hyperlipidemic and normolipidemic adults: a meta-analysis of randomized, controlled trials. European Journal of Clinical Nutrition, 53, 514-522.

Hanefeld, M., Fischer, S., Julius, U., Leonhardt, W., Schubert, E. \& Beckert, H. (1988). More exercise for the hyperlipidemic patients. Annals of Clinical Research, 20, 77-83.

Hansen, D., Dendale, P., Coninx, K., Vanhees, L., et al. (2017). The European Association of Preventive Cardiology Exercise Prescription in Everyday Practice and Rehabilitative Training (EXPERT) tool: A digital training and decision support system for optimized exercise prescription in cardiovascular disease. Concept, definitions and construction methodology. European Journal of Preventive Cardiology, 24, 1017-1031.

Hansen, D., Dendale, P., van Loon, L.J. \& Meeusen R. (2010). The impact of training modalities on the clinical benefits of exercise intervention in patients with cardiovascular disease risk or type 2 diabetes. Sports Medicine, 40, 921-940.

Harpal, S.B., Timao, L., and Nivedita, R. (2005). Prevention of cardiovascular diseases: Role of exercise, dietary interventions, obesity and smoking cessation. Experimental and Clinical Cardiology. Winter, 10(4), 229-249.

Harris, K.A. \& Holly, R.G. (1987). Physiological response to circuit weight training in border line hypertensive subjects. Medicine \& Science in Sports \& Exercise, 19, 246-252. 
Hartung, G.H., Foreyt, J.P., Mitchell, R.E., Vlasek, I., Gotto, A.M. JR. (1980). Relation of diet to high density lipoprotein cholesterol in middle-aged marathon runners, joggers, and inactive men. The New England Journal of Medicine, 302, 357-361.

Hartung, G.H., Squires, W.G. \& Gotto, A.M. (1981). Effect of exercise training on plasma highdensity lipoprotein cholesterol in coronary disease patients. American Heart Journal, 101, 181-184.

Haskell, W.L. (1980). Strenuous physical activity, treadmill exercise test response and plasma high -density lipoprotein cholesteror. The Lipid Research Clinic Program Prevalence Study. Circulation, 62, 53-61.

Haskell, W.L. (1984). Exercise-induced changes in plasma lipids and lipoproteins. Preventive Medicine, 13, 23-36.

Haskell, W.L., Lee, I.M., Pate, R.R., Powell, K.E., Blair, S.N, Franklin, B.A., et al. (2007). Physical activity and public health: updated recommendation for adults from the American College of Sports Medicine and the American Heart Association. Medicine \& Science in Sports \& Exercise, 39, 1423-1434.

Health and Safety Executive. Euro data statistics. 2011, London: Health and Safety Executive. Available at: http://www.hse.gov.uk/statistics/index.htm ( $\alpha v \alpha \kappa \tau \eta \dot{\theta \eta \kappa \varepsilon ~ A v ́ \gamma о v \sigma \tau o \varsigma ~}$ 2011).

Heidenreich, P.A., Trogdon, J.G., Khavjou, O.A., Butler, J., Dracup, K., Ezekowitz, M.D., Finkelstein, E.A., Hong, Y., Johnston, S.C., Khera, A., Lloyd-Jones, D.M., Nelson, S.A., Nichol, G., Orenstein, D., Wilson, P.W.F. \& Woo, Y.J. (2011). Forecasting the Future of Cardiovascular Disease in the United States. A Policy Statement From the American Heart Association. Circulation, 123, 933-944.

Hespel, P., Lijnen, P., Fagard, R., Van Hoof, R., Rosseneu, M. \& Amery, A. (1988). Changes in plasma lipids and apoproteins associated with physical training in in middle-aged sedentary men. American Heart Journal, 115, 786-792.

Hicks, A.L., Macdougall, J.D. \& Muckle, T.J. (1987). Acute changes in high-density lipoprotein cholesterol with exercise of different intensities. Journal of Applied Physiology, 63, 1956-1960.

Holloszy, J.O., Skinner, J.S., Toro, G. \& Cureton, T.K. (1964). Effects of a six month program of endurance exercise on serum lipids of middle-aged men. American Journal of Cardiology, 14, 753-760.

Hu, G., Barengo, N.C., Tuomilehto, J., Lakka, T.A., Nissinen, A. \& Jousilahti, P. (2004). Relationship of physical activity and body mass index to the risk of hypertension: a prospective study in Finland. Hypertension, 43, 25-30.

Hubert, H.B., Feinleib, M., Mcnamara, P.M. \& Casteli, W.P. (1983). Obesity as an independent risk factor for cardiovascular disease. A 26-year follow-up of participants in the Framingham Heart Study. Circulation, 67, 968-977.

Hunter, G.R., Bryan, D.R., Wetzstein, C.J., Zuckerman, P.A. \& Bamman, M.M. (2002). Resistance training and intra-abdominal adipose tissue in older men and women. Medicine \& Science in Sports \& Exercise, 34, 1023-1028.

Hunter, G.R., McCarthy, J.P. \& Bamman, M.M. (2004). Effects of resistance training on older adults. Sports Medicine, 34, 329-348. 
Huttunen, J.K., Lansimies, E., Voutilainen, E., Ehnholm, C., Hietanen, E., Penttila, I., Siitonen, O. \& Rauramaa, R. (1979). Effects of moderate physical exercise on serum lipoproteins. Circulation, 60, 1220-1229.

Ibanez, J., Izquierdo, M., Martinez-Labari, C., Ortega, F., Grijalba, A., Forga, L., et al. (2010). Resistance training improves cardiovascular risk factors in obese women despite a significative decrease in serum adiponectin levels. Obesity, 18, 535-541.

Igwebuike, A., Irving, B.A., Bigelow, M.L., Short, K.R., McConnell, J.P. \& Nair, K.S. (2008). Lack of dehydroepiandrosterone effect on a combined endurance and resistance exercise program in postmenopausal women. The Journal of Clinical Endocrinology \& Metabolism, 93, 534-538.

Iltis, P.W. (1984). Different running programs. Plasma lipids, apoproteins and lecithin: cholesterol acyltransferase in middle aged men. Annals of Sports Medicine, 2, 16-21.

Ishikawa-Takata, K., Ohta, T. \& Tanaka, H. (2003). How much exercise is required to reduce blood pressure in essential hypertensives: a dose-response study*. American Journal of Hypertension, 16, 629-633.

Jabekk, P.T., Moe, I.A., Meen, H.D., Tomten, S.E. \& Hostmark, A.T. (2010). Resistance training in overweight women on a ketogenic diet conserved lean body mass while reducing body fat. Nutrition \& Metabolism (Lond), 7, 17.

Jackson, A.S., Sui, X., Hebert, J.R., Church, T.S. \& Blair, S.N. (2009). Role of lifestyle and aging on the longitudinal change in cardiorespiratory fitness. Archives of Internal Medicine, 169, 1781-1787.

Jeemon, P., Harikrishnan, S., Sanjay, G., Sivasubramonian, S., Lekha, T.R., Padmanabhan, S., Tandon, N., Prabhakaran, D. (2017). A Programme of Lifestyle Intervention in Families for Cardiovascular risk reduction (PROLIFIC Study): design and rationale of a family based randomized controlled trial in individuals with family history of premature coronary heart disease. BMC Public Health, 17(1):10. doi: 10.1186/s12889-016-39286.

Jeffery, R.W., Wing, R.R., Sherwood, N.E. \& Tate, D.F. (2003). Physical activity and weight loss: does prescribing higher physical activity goals improve outcome? The American Journal of Clinical Nutrition, 78, 684-689.

Jennings, G., Nelson, L., Nestel, P., Elser, M., Korner, P., Burton, D. \& Bazelmans, B. (1986). The effects of changes in physical activity on major cardiovascular risk factors, hemodynamics, sympathetic function and glucose utilization in man. a controlled study of 4 levels of activity. Circulation, 73, 30-40.

Kahn, E.B., Ramsey, L.T., Brownson, R.C. et al. (2005). Task Force on Community Preventive Services - promoting physicazl activity. In: Zaza S, Briss PA, Harris KW, (eds.) The Guide to Community Preventive Services: What Works to Promote Health?, Atlanta (GA): Oxford University Press, pp. 80-113.

Kannel, W.B. \& Mcgee, D.L. (1979). Diabetes and cardiovascular disease. The Framingham study. Journal of the American Medical Association, 241, 2035-2038.

Kannel, W.B., Castelli, W.P., Mcnamara, P.M., Mckee, P.A. \& Feinleib, M. (1972). Role of blood pressure in the development of congestive heart failure. The Framingham study. The New England Journal of Medicine, 287, 781-787. 
Kaufman, F.L., Hughson, O.L. \& Shaman, J.P. (1987). Effect of exercise on recovery blood pressure in normotensive and hypertensive subjects. Medicine \& Science in Sports \& Exercise, 19, 17-20.

Kawano, M., Shono, N., Yoshimura, T., Yamaguchi, M., Hirano, T. \& Hisatomi, A. (2009). Improved cardio-respiratory fitness correlates with changes in the number and size of small dense LDL: randomized controlled trial with exercise training and dietary instruction. Internal Medicine (Tokyo, Japan), 48, 25-32.

Kelemen, M.H., Effron, M.B., Valenti, S.A. \& Stewart, K.J. (1990). Exercise training combined with antihypertensive drug therapy. Effects on lipids, blood pressure and left ventricular mass. Journal of the American Medical Association, 263, 2766-2771.

Kelley, G. \& Mcclellan, P. (1994) Antihypertensive effects of aerobic exercise. A brief metaanalytic review of randomized controlled trials. American Journal of Hypertension, 7, 115-119.

Kelley, G.A. \& Kelley, K.S. (2009). Impact of progressive resistance training on lipids and lipoproteins in adults: another look at a meta-analysis using prediction intervals. Preventive Medicine, 49, 473-475.

Kelley, G.A., Kelley, K.A. \& Vu Tran, Z. (2005). Aerobic exercise, lipids and lipoproteins in overweight and obese adults: a meta-analysis of randomized controlled trials. International Journal of Obesity (Lond), 29, 881-93.

Kelley, G.A., Kelley, K.S. \& Tran, Z.V. (2004). Aerobic exercise and lipids and lipoproteins in women: a meta-analysis of randomized controlled trials. Journal of Women's Health, 13(10), 1148-64.

Kelley, G.A., Kelley, K.S. \& Vu Tran, Z. (2005). Aerobic exercise, lipids and lipoproteins in overweight and obese adults: a meta-analysis of randomized controlled trials. International Journal of Obesity, 29, 881-893.

Kelley, G.A., Kelley, K.S., Roberts, S. \& Haskell, W. (2011). Efficacy of aerobic exercise and a prudent diet for improving selected lipids and lipoproteins in adults: a meta-analysis of randomized controlled trials. BMC Medicine, 9, 74-88.

Kennedy, C., Sprekeman, R.E. \& Markin, H.T. (1976). One-year graduated exercise program for men with angina pectoris. Mayo Clinic Proceedings, 51, 231-236.

Khan, K., McKay, H.A., Haapasalo, H., Bennell, K.L., Forwood, M.R., Kannus, P., et al. (2000). Does childhood and adolescence provide a unique opportunity for exercise to strengthen the skeleton? Journal of Science and Medicine in Sport, 3, 150-164.

Knowler, W.C., Barrett-Connor, E., Fowler, S.E., Hamman, R.F., Lachin, J.M., Walker, E.A. \& Nathan, D.M. (2002). Reduction in the incidence of type 2 diabetes with lifestyle intervention or metformin. The New England Journal of Medicine, 346, 393-403.

Kodama, S., Tanaka, S. \& Sito, K. (2007). Effect of aerobic exercise training on serum levels of high-density lipoprotein cholesterol. Archives of Internal Medicine, 167, 999-1008.

Kohl, H.W. 3rd, Gordon, N.F., Scott, C.B., Vaandrager, H. \& Blair, S.N. (1992). Musculoskeletal strength and serum lipid levels in men and women. Medicine \& Science in Sports \& Exercise, 24, 1080-1087.

Kokkinos, P.F. \& Fernall, B. (1999). Physical activity and high density lipoprotein cholesterol levels. Sports Medicine, 28, 307-314. 
Kokkinos, P.F. \& Hurley, B.F. (1990). Strength training and lipoprotein-lipids profile. a critical analysis and recommendations for further study. Sports Medicine, 9, 266-272.

Kokkinos, P.F. (1988). Effects of low and high repetition resistive training on lipoprotein-lipid profiles. Medicine \& Science in Sports \& Exercise, 20, 50-54.

Kokkinos, P.F., Holland, J.C., Narayan P., et al. (1995). Miles run per week and high-density lipoprotein cholesterol levels in healthy, middle-aged men: a dose-response relationship. Archives of Internal Medicine, 155, 415-20.

Kraus, W.E., Houmard, J.A., Duscha, B.D., et al. (2002). Effects of the amount and intensity of exercise on plasma lipoproteins. The New England Journal of Medicine, 347, 14831492.

Krieger, J.W. (2009). Single versus multiple sets of resistance exercise: a meta-regression. Journal of Strength and Conditioning Research, 23, 1890-1901.

Kuusi, T., Nikkila, E.A., Saarinen, P., Varjo, P. \& Laitinen, L.A. (1982). Plasma high-density lipoproteins HDL2, HDL3, and postheparin plasma lipases in relation to parameters of physical fitness. Atherosclerosis, 41, 209-219.

La Rosa, J.C., Cleary, P. \& Muesing, R.A. (1982). Effect of longterm moderate physical exercise on plasma lipoprotein. The national exercise heart disease project. Archives of Internal Medicine, 142, 2269-2274.

Lampman, R.M., Santiga, J.T., Hodge, M.F., Block, W.D., Flora, J.D. \& Bassett, D.R. (1977). Comparative effect of physical training and diet in normalizing serum lipids in men with type IV hyperlipolproteinema. Circulation, 55, 652-659.

Lampman, R.M., Santiga, J.T., Savage, P.J., Bassett, D.R., Hyndrick, C.R., Flora J.D., et al. (1985). Effect of exercise training on glucose tolerance in vivo insulin sensitivity, lipid and lipoprotein concentrations in middle-aged men with mild hypertriglyceridemia. Metabolism, 34, 205-211.

Larosa, J.C., Cleary, P., Muesing, R.A., Gorman, P., Hellerstein, H.K. \& Naughton, J. (1982). Effect of long-term moderate physical exercise in plasma lipoproteins. Archives of Internal Medicine, 142, 2269-2274.

Larson-Meyer, D.E., Redman, L., Heilbronn, L.K., Martin, C.K. \& Ravussin, E. (2010). Caloric restriction with or without exercise: the fitness versus fatness debate. Medicine \& Science in Sports \& Exercise, 42, 152-159.

Latimer-Cheung, A.E., Pilutti, L.A., Hicks, A.L., Martin Ginis, K.A., Fenuta, A.M., MacKibbon, K.A., \& Motl, R.W. 2013. Effects of exercise training on fitness, mobility, fatigue, and health-related quality of life among adults with multiple sclerosis: a systematic review to inform guideline development. Archives of Physical Medicine and Rehabilitation, 94, 1800-1828.

Lee, D.C., Sui, X., Church, T.S., Lavie, C.J., Jackson, A.S. \& Blair, S.N. (2012). Changes in fitness and fatness on the development of cardiovascular disease risk factors hypertension, metabolic syndrome, and hypercholesterolemia. Journal of the American College of Cardiology, 59, 665-672.

Lee, I., Chung-Cheng, C.I. \& Paffenbarger, R.S. (1995). Exercise intensity and longevity in men. the Harvard alumni health study. Journal of the American Medical Association, 273, 1179-1184. 
Lee, I.M. \& Skerrett, P.J. (2001). Physical activity and all-cause mortality: what is the doseresponse relation? Medicine \& Science in Sports \& Exercise, 33(6 Suppl):S459-71, discussion S493-4.

Leon, A.S. \& Sanchez, O.A. (2001). Response of blood lipids to exercise training alone or combined with dietary intervention. Medicine \& Science in Sports \& Exercise, 33(6 suppl), S502-S515, discussion S528-S529.

Leon, A.S., Agre, J., Mcnally, C., Bell, C., Neibleing, M., Grimm, R. \& Hunninghake, D.B. (1984). Blood lipid effects of antihypertensive therapy. A double-blind comparison of the effects of methyldopa and propranonol. Journal of Clinical Pharmacology, 24, 209217.

Leon, A.S., Rice, T., Mandel, S., et al. (2000). Blood lipid response to 20 weeks of supervised exercise in a large biracial population: the HERITAGE Family Study. Metabolism, 49, 513-520.

Leutholtz, B.C., Keyser, R.E., Heusner, W.W., Wendt, V.E. \& Rosen, L. (1995). Exercise training and severe caloric restriction: effect on lean body mass in the obese. Archives of Physical Medicine and Rehabilitation, 76, 65-70.

Levine, S.A. \& Lown, B. (1952). Armchair treatment of acute coronary thrombosis. Journal of the American Medical Association 148, 1365-1369.

Li, Y., Hanssen, H., Cordes, M., Rossmeissl, A., Endes, S., \& Schmidt-Trucksäss, A. (2015). Aerobic, resistance and combined exercise training on arterial stiffness in normotensive and hypertensive adults: A review. European Journal of Sport Science, 15, 443-57. doi: 10.1080/17461391.2014.955129. Epub 2014 Sep 24.

Liebson, P.R., Grandits, G., Prineas, R., Dianzumba, S., Flack, J.M., Cuttler, J.A., Grimm, R. \& Stamler, J. (1993). Echocardiografic correlates of left ventricular structure among 844 mildly hypertensive men and women in the Treatment of Mild Hypertension Study (TOMHS). Circulation, 87, 476-486.

Lipson, L.C., Bonow, R.O., Schaefer, E.J., Brewer, H.B. \& Lindgren, F.T. (1980). Effect of exercise conditioning on plasma high-density lipoproteins and other lipoproteins. Atherosclerosis, 37, 529-538.

Longhurst, J.C. \& Mitchell, H.H. (1983). Does endurance training benefit the cardiovascular system? Journal of Cardiovascular Medicine, 8, 227-236.

Lochen, M.-L. \& Rasmussen, K. (1992). The Tromsø Study. physical fitness, self reported physical activity, and their relationship to other coronary risk factors. Journal of Epidemiology and Community Health, 46, 103-107.

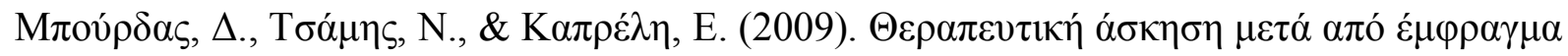

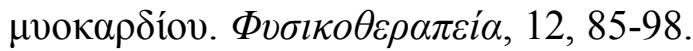

Macknight, J.M. (2003). Exercise considerations in hypertension, obesity, and dyslipidemia. Clinics in Sports Medicine, 22(1), 101-21, vii.

Maesta, N., Nahas, E.A., Nahas-Neto, J., Orsatti, F.L., Fernandes, C.E., Traiman, P., et al. (2007). Effects of soy protein and resistance exercise on body composition and blood lipids in postmenopausal women. Maturitas, 56, 350-358.

Magnus, P., Borresen, A.L., Opstad, P.K., Bugge, J.F. \& Berg, K. (1984). Increase in the ratio of serum levels of apoprotein A-I and A-II duirng prolonged physical strain and calorie deficiency. European Journal of Applied Physiology, 53, 21-24. 
Mahar, M.T., Murphy, S.K., Rowe, D.A., et al. (2006). Effects of a classroom-based program on physical activity and ontask behaviour. Medicine \& Science in Sports \& Exercise, 38, 2086-2094.

Manninen, V., Elo, M.O., Frick, M.H., Haapa, K., Heinonen, O.P., Heinsalmi, P., Helo P., Huttunen, J.K., Kaitaniemi, P. \& Koskinen, P. (1988). Lipid alterations and dicline in the incidence of coronary heart disease in the Helsinki Heart Study. Journal of the American Medical Association, 260: 641-651.

Manning, J.M. (1991). Effects of a resistive training program on lipoprotein-lipid levels in obese women. Medicine \& Science in Sports \& Exercise, 23, 1222-1226.

Marques, E., Carvalho, J., Soares, J.M., Marques, F. \& Mota, J. (2009). Effects of resistance and multicomponent exercise on lipid profiles of older women. Maturitas, 63, 84-88.

Marti, B., Knobloch, M., Riesen, W.F. \& Howald, H. (1991). Fifteen years changes in exercise, aerobic power, abdominal fat, and serum lipids in runners and controls. Medicine \& Science in Sports \& Exercise, 23, 115-122.

Marti, B., Suter, E., Riesen, W.F., Tschopp, A., Wanner, H.U. \& Gutzwiller, F. (1990). Effects of long-term, self-monitored exercise on the serum lipoprotein and apolipoprotein profile in middle-aged men. Atherosclerosis, 81, 19-31.

Mcdonough, J.R., Kusumi, F. \& Bruce, R.A. (1970). Variations in maximal oxygen intake with physical activity in middle-aged men. Circulation, 41, 743-751.

McGowan, C.L., Levy, A.S., Millar, P.J., Guzman, J.C., Morillo, C.A., McCartney, N. \& Macdonald, M.J. (2006). Acute vascular responses to isometric handgrip exercise and effects of training in persons medicated for hypertension. American Journal of Physiology-Heart and Circulatory Physiology, 291, 28.

McGuigan, M.R., Tatasciore, M., Newton, R.U. \& Pettigrew, S. (2009). Eight weeks of resistance training can significantly alter body composition in children who are overweight or obese. Journal of Strength and Conditioning Research, 23, 80-85.

McMurray, R.G., Ainsworth, B.E., Harrell, J.S., Griggs, T.R. \& Williams, O.D. (1998). Is physical activity or aerobic power more influential on reducing cardiovascular disease risk factors? Medicine \& Science in Sports \& Exercise, 30, 1521-1529.

Melish, J., Bronstein, D. \& Gross, R. (1978). Effect of exercise training in type II hyperlipoproteinemia. Circulation, 57, 38.

Meredith, I.T., Friberg, P., Jennings, G.L., Dewar, E.M., Fazio, V.A., Lambert G.W. \& Esler, M.D. (1991). Exercise training lowers resting renal but not cardiac sympathetic activity in humans. Hypertension, 18, 575-582.

Mestek, M.L., Garner, J.C., Plaisance, E.P., Taylor, J.K., Alhassan, S. \& Grandjean, P.W. (2006). Blood lipid responses after continuous and accumulated aerobic exercise. International Journal of Sport Nutrition Exerc Metab, 16, 245-254.

Miller, N.E., Hammett, F., Saltissi, S., Rao, S., Van Zeller, H., Coltart, J. \& Lewis, B. (1981). Relation of angiographically defined coronary artery disease to plasma lipoprotein subfractions and apolipoproteins. British Medical Journal, 282, 1741-1744.

Miller, W.C., Koceja, D.M. \& Hamilton, E.J. (1997). A meta-analysis of the past 25 years of weight loss research using diet, exercise or diet plus exercise intervention. International Journal of Obesity and Related Metabolic Disorders, 21, 941-947. 
Missault, L.H., Duprez, D.A., Brandt, A.A. (1993). Exercise performance and diastolic filling in essential hypertension. Blood Press, 2, 284-288.

Montoye, H.J., Block, W.D. \& Gayle, R. (1978). Maximal oxygen intake and blood lipids. Journal of Chronic Diseases, 31, 111-118.

Mooss, A., Gordon, N.F. (2001). Conseptual basis for coronary artery disease, risk factor assessment in clinical practise. In. Lippincot Williams \& Wilkins (eds.) ACSM's resource manual for guidelines for exercise testing and prescription ( $4^{\text {th }} \mathrm{ed}$ ). Philadelfia, pp. 3-16.

Mosher P.E., Ferguson M.A. \& Arnold R.O. (2005). Lipid and lipoprotein changes in premenstrual women following step aerobic dance training. International Journal of Sports Medicine, 26, 669-674.

Murray, N.G., Low, B.J., Hollis, C., et al. (2007). Coordinated school health programs and academic achievement: a systematic review of the literature. Journal of School Health, 77, 589-600.

Nakamura, N., Uzawa, H., Maeda, H. \& Inomoto, T. (1983). Physical fitness. Its contribution to serum high-density lipoprotein. Atherosclerosis, 48, 173.

NIH Consensus Conference (1996). Physical activity and cardiovascular health. Journal of the American Medical Association, 276: 241-246.

Nindl, B.C., Barnes, B.R., Alemany, J.A., Frykman, P.N., Shippee, R.L. \& Friedl, K.E. (2007). Physiological consequences of U.S. Army Ranger training. Medicine \& Science in Sports \& Exercise, 39, 1380-1387.

Oguma, Y. \& Shinoda-Tagawa, T. (2004). Physical activity decreases cardiovascular disease risk in women: review and meta-analysis. American Journal of Preventive Medicine, 26(5), 407-18.

Orsatti, F.L., Nahas, E.A., Maesta, N., Nahas-Neto, J. \& Burini, R.C. (2008). Plasma hormones, muscle mass and strength in resistance-trained postmenopausal women. Maturitas, 59, 394-404.

Oscai, L.B., Patterson, J.A. \& Bogard, D.L. (1972). Normalization of serum triglycerides and lipoprotein electrophoretic pattern by exercise. American Journal of Cardiology, 30, 775-780.

Paffenbarger, R.S. JR., Wing, A.L., Hyde, R.T. \& Jung, D.L. (1983). Physical activity and incidence of hypertension in college alumni. American Journal of Epidemiology, 117, 245-257.

Paffenbarger, R.S., Hyde, R.T., Wing, A.L. \& Hsieh, C.C. (1986). Physical activity, all-cause mortality, and longetivity of college alumni. The New England Journal of Medicine, $314,605-613$.

Pagonas, N., Vlatsas, S., Bauer, F., Seibert, F.S., Zidek, W., Babel, N., Schlattmann, P., \& Westhoff, T.H. (2017). Aerobic versus isometric handgrip exercise in hypertension: a randomized controlled trial. Journal of Hypertension, 35, 2199-2206.

Park, Y.M., Sui, X., Liu, J., et al. (2015). The effect of cardiorespiratory fitness on agerelated lipids and lipoproteins. Journal of the American College of Cardiology, 65, 2091-2100.

Pate, R.R., Pratt, M., Blair, S.N., Haskell, W.L., Macera, C.A., Bouchard, C., Buchner, D., Ettinger, W., Heath, G.W., King, A.C., Kriska, A., Leon, A.S., Marcus, B.H., Morris, J., Paffenbarger, R.S., Patrick, K., Pollock, M.L., Rippe, J.M., Sallis, J. \& Wilmore, J.H. 
(1995). Physical activity and public health. a recommendation from the Centers for Disease Control and Prevention and the American College of Sports Medicine. Journal of the American Medical Association, 273, 402-407.

Pattyn, N., Cornelissen, V.A., Eshghi, S.R. \& Vanhees, L. (2013). The effect of exercise on the cardiovascular risk factors constituting the metabolic syndrome: a meta-analysis of controlled trials. Sports Medicine, 43, 121-133.

Pavlou, K.N., Krey, S. \& Steffee, W.P. (1989). Exercise as an adjunct to weight loss and maintenance in moderately obese subjects. The American Journal of Clinical Nutrition, 49, 1115-1123.

Payne, W.A. \& Hahn, D.B. (1992). Understanding your health $4^{\text {th }}$ edition. St.Luis, Missuri: Mosby-Year Book.

Pekkanen, J., Linn, S., Heiss, G., Suchindran, C.M., Leon, A., Rifkind, B.M. \& Tyroler, H.A. (1990). Ten year mortality from cardiovascular disease in relation to cholesterol level among men with and without pre-existing cardiovascular disease. The New England Journal of Medicine, 322, 1700-1707.

Peltonen, P., Marniemi, J., Hietanen, E., Vuori, I. \& Ehnholm, C. (1981). Changes in serum lipids, lipoproteins and heparin-releasable lipolytic enzymes during moderate physical training in man. Metabolism, 30, 518-526.

Pescatello, L.S., MacDonald, H.V., Lamberti, L. \& Johnson, B.T. (2015). Exercise for Hypertension: A Prescription Update Integrating Existing Recommendations with Emerging Research. Current Hypertension Reports, 17, 87

Piepoli, M.F., Hoes, A.W., Agewall, S., et al. (2016). European guidelines on cardiovascular disease prevention in clinical practice. European Journal of Preventive Cardiology, 23, NP1-NP96.

Pitsavos, C., Panagiotakos, D.B., Tambalis, K.D., Chrysohoou, C., Sidossis, L.S., Skoumas J., et al. (2009). Resistance exercise plus to aerobic activities is associated with better lipids' profile among healthy individuals: the ATTICA study. Quarterly Journal of Medicine, 102, 609-616.

Pollock, M.L., Franklin, B.A., Balady, G.J., Chaitman, B.L., Fleg, J.L., Fletcher, B., Limacher, M., Pina, I.L., Stein, R.A., Williams, M. \& Bazzarre, T. (2000). AHA Science Advisory. Resistance exercise in individuals with and without cardiovascular disease: benefits, rationale, safety, and prescription: an advisory from the Committee on Exercise, Rehabilitation, and Prevention, Council on Clinical Cardiology, American Heart Association; Position paper endorsed by the American College of Sports Medicine. Circulation, 101, 828-833.

Powell, K.E., Thompson, P.D., Caspersen, C.J. \& Kendrick, J.S. (1987). Physical activity and the incidence of coronary heart disease. Annual Review of Public Health, 8, 253-287.

Pulfrey, S.M. \& Jones, P.J. (1996). Energy expenditure and requirement while climbing above 6,000 m. Journal of Applied Physiology, 81, 1306-1311.

Raz, I., Rosenblit, H. \& Kark, J.D. (1988). Effect of moderate exercise on serum lipids in young men with low high density lipoprotein cholesterol. Arteriosclerosis, 8, 245-251.

Reiner, Z`., Catapano, A.L., De Backer, G., Graham, I., Taskinen, M.R., Wiklund, O., et al. (2011). ESC/EAS Guidelines for the management of dyslipidaemias: the Task Force for the management of dyslipidaemias of the European Society of Cardiology (ESC) and the European Atherosclerosis Society (EAS). European Heart Journal, 32, 1769-1818. 
Richard, E., Jongstra, S., Soininen, H., Brayne, C., Moll, van Charante, EP., Meiller, Y., van der Groep, B., Beishuizen, C.R., Mangialasche, F., Barbera, M., Ngandu, T., Coley, N., Guillemont, J., Savy, S., Dijkgraaf, M.G., Peters, R.J., van Gool, W.A., Kivipelto, M., Andrieu, S. (2016). Healthy Ageing Through Internet Counselling in the Elderly: the HATICE randomised controlled trial for the prevention of cardiovascular disease and cognitive impairment. British Medical Journal Open, 10, 6(6), e010806.

Riddell, M.A., Edwards, N., Thompson, S.R., Bernabe-Ortiz, A., Praveen, D., Johnson, C., Kengne, A.P., Liu, P., McCready, T., Ng E., Nieuwlaat, R., Ovbiagele, B., Owolabi, M., Peiris, D., Thrift, A.G., Tobe, S., Yusoff, K. (2017). GACD Hypertension Research Programme. Developing consensus measures for global programs: lessons from the Global Alliance for Chronic Diseases Hypertension research program. Global Health 15,13(1), 17.

Ross, R. \& Rissanen, J. (1994). Mobilization of visceral and subcutaneous adipose tissue in response to energy restriction and exercise. The American Journal of Clinical Nutrition, $60,695-703$.

Ross, R., Rissanen, J., Pedwell, H., Clifford, J. \& Shragge, P. (1996). Influence of diet and exercise on skeletal muscle and visceral adipose tissue in men. Journal of Applied Physiology, 81, 2445-2455.

Sanderson, B., Askew, C., Stewart, I., et al. (2006). Short-term effects of cycle and treadmill training on exercise tolerance in peripheral arterial disease. Journal of Vascular Surgery, 44, 119-127.

Saris, W.H., Blair, S.N., van Baak, M.A., Eaton, S.B., Davies, P.S., Di P.L., et al. (2003). How much physical activity is enough to prevent unhealthy weight gain? Outcome of the IASO 1st Stock Conference and consensus statement. Obesity Reviews, 4, 101-114.

Savage, M.P., Petratis, M.M., Thomson, W.H., Berg, K., Smith, J.L. \& Sady, S.P. (1986). Exercise training effects on serum lipids of prepubescent boys and adult men. Medicine \& Science in Sports \& Exercise, 18, 197-204.

Schaefer, E.J. \& Lichtenstein, A.H, Lamon-Fava, S., Mcnamara, J.R., Schaefer, M.M., Rasmussen, H. \& Ordovas, J.M. (1995). Body weight and low density lipoprotein cholesterol changes after consumption of a low fat ad libitum diet. Journal of the American Medical Association, 274, 1450-1455.

Schjerve, I.E., Tyldum, G.A., Tjonna, A.E., Stolen, T., Loennechen, J.P., Hansen, H.E., et al. (2008). Both aerobic endurance and strength training programmes improve cardiovascular health in obese adults. Clinical Science, 115, 283-293.

Sgro, M., McGuigan, M.R., Pettigrew, S. \& Newton, R.U. (2009). The effect of duration of resistance training interventions in children who are overweight or obese. Journal of Strength and Conditioning Research, 23, 1263-1270.

Shaibi, G.Q., Cruz, M.L., Ball, G.D., Weigensberg, M.J., Salem, G.J., Crespo, N.C., et al. (2006). Effects of resistance training on insulin sensitivity in overweight Latino adolescent males. Medicine \& Science in Sports \& Exercise, 38, 1208-1215.

Sharman, J.E. \& Stowasser, M. (2009). Australian association for exercise and sports science position statement on exercise and hypertension. Journal of Science and Medicine in Sport, 12, 252-257.

Sharman, J.E., La Gerche, A. \& Coombes, J.S. (2015). Exercise and Cardiovascular Risk in Patients With Hypertension. American Journal of Hypertension, 28, 147-58. 
Shaw, I. \& Shaw, B.S. (2008). Relationship between resistance training and lipoprotein profiles in sedentary male smokers. Cardiovascular Journal of Africa, 19, 194-197.

Simopoulos, A.P. \& Van Itallie, T.B. (1984). Body weight, health and longevity. Annals of Internal Medicine, 100: 285-295.

Slentz, C.A., Houmard, J.A. \& Kraus, W.E. (2009). Exercise, abdominal obesity, skeletal muscle, and metabolic risk: evidence for a dose response. Obesity, 17(Suppl 3), S27S33.

Sothern, M.S., Loftin, J.M., Udall, J.N., Suskind, R.M., Ewing, T.L., Tang, S.C., et al. (1999). Inclusion of resistance exercise in a multidisciplinary outpatient treatment program for preadolescent obese children. Southern Medical Journal, 92, 585-592.

Stamler, J., Neaton, J. \& Wentworth, D. (1993). Blood pressure and risk of fatal coronary heart disease. Hypertension, 13, 2-12.

Steele, R.M., Brage, S., Corder, K., Wareham, N.J. \& Ekelund, U. (2008). Physical activity, cardiorespiratory fitness, and the metabolic syndrome in youth. Journal of Applied Physiology, 105, 342-351.

Stein, R.A., Michielli, D.W., Glantz, M.D., Sardy, H., Cohen, A., Goldberg, N. \& Brown, C.D. (1990). Effects of different exercise training intensities on lipoprotein cholesterol fractions in healthy middle aged men. American Heart Journal, 119(2 pt 1), 277-283.

Stone M.H. (1983). Cardiovascular responces to short term Olympic style weight training in young men. Canadian Journal of Applied Sport Sciences, 8, 134-139.

Sui, X., Sarzynski, M.A., Lee, D.C. \& Kokkinos, P.F. (2017). Impact of Changes in Cardiorespiratory Fitness on Hypertension, Dyslipidemia and Survival: An Overview of the Epidemiological Evidence. Progress in Cardiovascular Diseases, 60, 56-66.

Sumimoto, T., Hamada, M., Muneta, S., Shigematsu, Y., Fujiwara, Y., Sekiya, M., Kazatani, Y. \& Hiwada, K. (1991). Influence of age and severity of hypertension on blood pressure response to isometric handgrip exercise. Journal of Human Hypertension, 5, 399-403.

Sutherlan, W. \& Woodhouse, S. (1980). Physical activity and plasma lipid concentrations in men. Atherosclerosis, 37, 285-289.

Sutherland, W.H.F., Nye, E.R. \& Wodhouse, S.P. (1983). Red blood cell cholesterol levels, plasma cholesterol esterification rate and serum lipid and lipoproteins in men with hypercholesterolaemia and normal men during 16 weeks physical training. Atherosclerosis, 47, 145-157

Tambalis, K., Panagiotakos, D.B., Kavouras, S.A. \& Sidossis, L.S. (2009). Responses of blood lipids to aerobic, resistance, and combined aerobic with resistance exercise training: a systematic review of current evidence. Angiology, 60, 614-632.

Taylor, A.C., McCartney, N., Kamath, M.V. \& Wiley, R.L. (2003). Isometric training lowers resting blood pressure and modulates autonomic control. Medicine \& Science in Sports \& Exercise, 35, 251-256.

Taylor, R.S., Brown, A., Ebrahim, S., Jolliffe, J., Noorani, H., Rees, K., Skidmore, B., Stone, J.A., Thompson, D.R., \& Oldridge, N. (2004). Exercise-based rehabilitation for patients with coronary heart disease: systematic review and meta-analysis of randomized controlled trials. American Journal of Medicine, 116(10), 682-92 
Teixeira, P.J., Going, S.B., Houtkooper, L.B., Metcalfe, L.L., Blew, R.M., Flint-Wagner, H.G., et al. (2003). Resistance training in postmenopausal women with and without hormone therapy. Medicine \& Science in Sports \& Exercise, 35, 555-562.

The Sixth Report Of The Joint National Committee On Prevention, Detection, Evaluation And Treatment Of High Blood Pressure (1997). National High Blood Pressure Education Program. National Institutes of Health. National Heart, Lung and Blood Istitute. NIH Publication, 98-4080.

Thomas, T.R. \& Lafontaine, T.P. (2001). Exercise, Nutritional Strategies and Lipoproteins, in. Lippincot Williams \& Wilkins (eds.), ACSM's Resource Manual for Guidelines Exercise Testing and Prescription, $4^{\text {th }}$ ed. Philadelfia, pp 308-316.

Toth, M.J., Beckett, T. \& Poehlman, E.T. (1999). Physical activity and the progressive change in body composition with aging: current evidence and research issues. Medicine \& Science in Sports \& Exercise, 31(Suppl 11), S590-S596.

Tran, Z.V. \& Weltman, A. (1985). Differential effects of exercise on serum lipid and lipoprotein levels seen with changes in body weight. A meta-analysis. Journal of the American Medical Association, 254, 919-924.

Tran, Z.V. (1983). The effects of exercise on blood lipids and lipoproteins. A meta-analysis of studies. Medicine \& Science in Sports \& Exercise, 15, 393-402.

Tran, Z.V., Weltman, A., Glass, G.V. \& Mood, D.P. (1998). The effects of exercise on blood lipids and lipoproteins: a meta-analysis of studies. Medicine \& Science in Sports \& Exercise, 30, 1521-1529.

Tresierras, M.A. \& Balady, G.J. (2009). Resistance training in the treatment of diabetes and obesity: mechanisms and outcomes. Journal of Cardiopulmonary Rehabilitation and Prevention, 29, 67-75.

Treuth, M.S., Hunter, G.R., Kekes-Szabo, T., Weinsier, R.L., Goran, M.I. \& Berland, L. (1995). Reduction in intra-abdominal adipose tissue after strength training in older women. Journal of Applied Physiology, 78, 1425-1431.

Treuth, M.S., Ryan, A.S., Pratley, R.E., Rubin, M.A., Miller, J.P., Nicklas, B.J., et al. (1994). Effects of strength training on total and regional body composition in older men. Journal of Applied Physiology, 77, 614-620.

Tucker, L.A. (1994). Strength training and hypercholesterolemia [abstract]. Research Quarterly for Exercise and Sport, 65, A54-A55.

Uthman, O.A., Hartley, L., Rees K., Taylor, F., Ebrahim S. \& Clarke, A. (2015). Multiple risk factor interventions for primary prevention of cardiovascular disease in low- and middle-income countries. The Cochrane Database of Systematic Reviews, 4,(8), CD011163.

van Aggel-Leijssen, D., Saris, W.H.M., Wagenmakers, A.J.M., Senden, J.M. \& van Baak, M.A. (2002). Effect of exercise training at different intensities on fat metabolism of obese men. Journal of Applied Physiology, 92, 1300-1309.

van der Heijden, G.J., Wang, Z.J., Chu, Z., Toffolo, G., Manesso, E., Sauer, P.J., et al. (2010). Strength exercise improves muscle mass and hepatic insulin sensitivity in obese youth. Medicine \& Science in Sports \& Exercise, 42, 1973-1980.

Van Itallie, T.B. (1985). Health implications of overweight and obesity in the United States. Annals of Internal Medicine, 103(6 pt 2), 983-988. 
van Sluijs, E.M., McMinn, A.M. \& Griffin, S.J. (2007). Effectiveness of interventions to promote physical activity in children and adolescents: systematic review of controlled trials. British Medical Journal, 335, 703.

Vanhees, L., De Sutter, J., Geladas, N., Doyle, F., Prescott, E., Cornelissen, V., et al. (2012a). Importance of characteristics and modalities of physical activity and exercise in the management of cardiovascular health within the general population. Recommendations from the EACPR (Part I). European Journal of Cardiovascular Prevention and Rehabilitation, 19, 670-686

Vanhees, L., Geladas, N., Hansen, D., et al. (2012b). Importance of characteristics and modalities of physical activity and exercise in the management of cardiovascular health in individuals with cardiovascular risk factors. Recommendations from the European Association for Cardiovascular Prevention and Rehabilitation (Part II). European Journal of Preventive Cardiology, 19, 1005-1033.

Villareal, D.T., Apovian, C.M., Kushner, R.F. \& Klein, S. (2005). Obesity in older adults: technical review and position statement of the American Society for Nutrition and NAASO, The Obesity Society. The American Journal of Clinical Nutrition, 82, 923934.

Villareal, D.T., Banks, M., Sinacore, D.R., Siener, C. \& Klein, S. (2006). Effect of weight loss and exercise on frailty in obese older adults. Archives of Internal Medicine, 166, 860866.

Wallace, R.B. \& Anderson, R.A. (1987). Blood lipids, lipid-related measures, and risk of atherosclerotic cardiovascular disease. Epidemiologic Reviews, 9, 95-119.

Wareham, N. (2007). Physical activity and obesity prevention. Obesity Reviews, 8(Suppl 1), $109-114$

Wen, C.P., Wai, J.P., Tsai, M.K., Yang, Y.C., Cheng, T.Y., Lee, M.C., Chan, H.T., Tsao, C.K., Tsai, S.P. \& Wu, X. (2011). Minimum amount of physical activity for reduced mortality and extended life expectancy: a prospective cohort study. Lancet, 378, 1244-1253.

Whatley, J.E., Gillespie, W.J., Honig, J., Walsh, M.J., Blackburn, A.L. \& Blackburn, G.L. (1994). Does the amount of endurance exercise in combination with weight training and a very-low-energy diet affect resting metabolic rate and body composition? The American Journal of Clinical Nutrition, 59, 1088-1092.

Whelton, S.P., Chin, A., Xin, X. \& He, J. (2002). Effect of aerobic exercise on blood pressure: a meta-analysis of randomized, controlled trials. Annals of Internal Medicine, 136, 493503

Wijndaele, K., Duvigneaud, N., Matton, L., Duquet, W., Thomis, M., Beunen G., et al. (2007). Muscular strength, aerobic fitness, and metabolic syndrome risk in Flemish adults. Medicine \& Science in Sports \& Exercise, 39, 233-240.

Wilkins, E., Wilson, L., Wickramasinghe, K., Bhatnagar, P., Leal, J., Luengo-Fernandez, R., Burns, R., Rayner, M., \& Townsend, N. (2017). European Cardiovascular Disease Statistics 2017. European Heart Network, Brussels.

Williams, P.T, Wood, P.D., Haskell, W.L. \& Vranizan, K. (1982). The effects of running mileage and duration on plasma lipoprotein levels. Journal of the American Medical Association, 247, 2674-2679.

Williams, P.T. (1998). Relationships of heart disease risk factors to exercise quantity and intensity. Archives of Internal Medicine, 158, 237-245. 
Williams, P.T. (2008). Vigorous exercise, fitness and incident hypertension, high cholesterol, and diabetes. Medicine \& Science in Sports \& Exercise, 40, 998-1006.

Wilmore, J.H. (1976). Strength endurance, BMR and body composition changes during circuit weight training. Medicine \& Science in Sports \& Exercise, 8, 59-60.

Wirth, A., Diehm, C., Hanel, W., Welte, J. \& Vogel, I. (1985). Training-induced changes in serum lipids, fat tolerance, and adipose tissue metabolism in patients with hypertriglyceridemia. Atherosclerosis, 54, 263-271.

Wolfe, B.L., LeMura, L.M. \& Cole, P.J. (2004). Quantitative analysis of single- vs. multipleset programs in resistance training. Journal of Strength and Conditioning Research, 18, $35-47$.

Wood, P.D., Haskell, W.L., Blair, S.N., Williams, P.T., Krauss, R.M., Lindgren, F.T., et al. (1983). Increased exercise level plasma lipoprotein concentrations: a one-year, randomized, controlled study in sedentary middle-aged men. Metabolism, 32, 31-39.

Wood, P.D., Stefanick, M.L., Williams, P.T. \& Haskell, W.L. (1991). The effects on plasma lipoproteins of prudent weight-reducing diet, with and without exercise, in over-weight men and women. The New England Journal of Medicine, 325, 461-466.

World Health Organization (1997). World Health Report 1997. Geneva.

World Health Organization. Projections of mortality and burden of disease, 2002-2030. Available at: http:// www.who.int/healthinfo/global_burden_disease/projections/

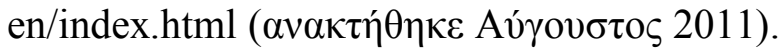

Young, D.R. \& Steinhardt, M.A. (1993). The importance of physical fitness versus physical activity for coronary artery disease risk factors. a cross-sectional analysis. Research Quarterly for Exercise and Sport, 4, 377-384.

Yu, C.C., Sung, R.Y., So, R.C., Lui, K.C., Lau, W., Lam, P.K., et al. (2005). Effects of strength training on body composition and bone mineral content in children who are obese. Journal of Strength and Conditioning Research, 19, 667-672. 